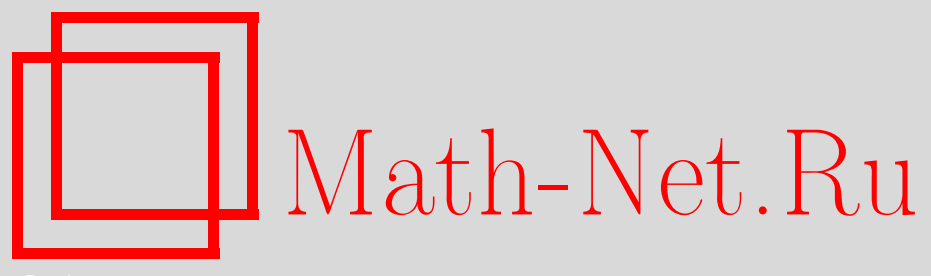

Е. П. Долженко, Оценки модулей непрерывности конформных отображений областей вблизи их достижимых граничных дуг, Матем. сб., 2011, том 202, номер 12, 57106

DOI: https://doi.org/10.4213/sm7648

Использование Общероссийского математического портала Math-Net.Ru подразумевает, что вы прочитали и согласны с пользовательским соглашением http: //www . mathnet.ru/rus/agreement

Параметры загрузки:

IP : 35.173 .219 .149

26 апреля 2023 г., 09:30:53

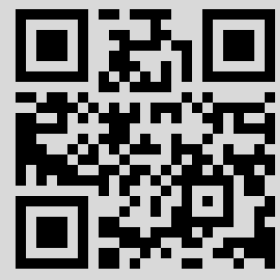




\title{
Е. П. Долженко \\ Оценки модулей непрерывности конформных отображений областей вблизи их достижимых граничных дуг
}

\begin{abstract}
Работа содержит оценки обычных модулей непрерывности $\omega(f, \bar{G}, \delta)$ конформных отображений $w=f(z)$ одной ограниченной односвязной области $G$ с произвольной жордановой границей на другую ограниченную односвязную область с произвольной жордановой границей в зависимости от "качества" этих границ. При этом качество жордановой кривой (разомкнутой дуги или замкнутого контура) в общем случае характеризуется ее модулем колебания, а в случае конечности ее длины - также и более чувствительным ее модулем спрямляемости (эти чисто метрические понятия введены автором в 1996 г.). Для односвязных областей произвольной природы доказаны соответствующие теоремы о поведении их конформных отображений вблизи открытых достижимых граничных дуг этих областей.
\end{abstract}

Библиография: 18 названий.

Ключевые слова: однолистное конформное отображение, достижимая граничная дуга односвязной области, модуль непрерывности, модуль колебания, модуль спрямляемости.

\section{Введение}

Теорема Б. Римана утверждает, что всякую расположенную на комплексной плоскости односвязную область $G$ с непустой границей $\partial G$, не вырожденной в единственную точку, можно с помощью некоторой однозначной и однолистной аналитической функции $w=f(z)$ комплексного переменного конформно отобразить на любую другую заранее заданную односвязную область $Q$ также с непустой и не вырожденной в точку границей $\partial Q$. При этом по теореме K. Каратеодори если $\partial G$ и $\partial Q$ суть жордановы контуры, то $f(z)$ непрерывно продолжается на $\partial G$ до гомеоморфизма замыкания $\bar{G}$ области $G$ на замыкание $\bar{Q}$ области $Q$. Эта замечательная теорема имеет чисто качественный характер, поскольку она не указывает каких-либо количественных зависимостей метрических свойств (например, гладкостных в каком-либо смысле) отображающей функции $f(z)$ от метрико-геометрических свойств кривых $\partial G$ и $\partial Q$. В случае обычной гладкости этих кривых вопрос о таких связях решают (при некоторых дополнительных условиях) известные теоремы О. Д. Келога (см. [1]), С. Е. Варшавского (см. [2], [3]) и др. В [3] рассмотрены также конформные отображения $w=f(z)$ единичного круга $D:=\{z:|z|<1\}$ на области $Q$

Работа выполнена при поддержке Российского фонда фундаментальных исследований (грант № 08-01-00648a). 
с простейшими негладкими границами $L=\partial Q$, а именно, такими жордановыми спрямляемыми кривыми $L$, что для любых двух точек $w^{\prime}, w^{\prime \prime} \in L$ длина $\left|L\left(w^{\prime}, w^{\prime \prime}\right)\right|:=\operatorname{mes}_{1}\left(L\left(w^{\prime}, w^{\prime \prime}\right)\right)$ кратчайшей из двух дуг контура $L$, соединяющих эти две точки, по величине соизмерима с длиной хорды этой дуги, т.е. $\left|w^{\prime}-w^{\prime \prime}\right| \leqslant\left|L\left(w^{\prime}, w^{\prime \prime}\right)\right| \leqslant c\left|w^{\prime}-w^{\prime \prime}\right|$, где $c=c(L)>1$ не зависит от $w^{\prime}$ и $w^{\prime \prime}$ (такие кривые $L$ называют кривыми М. А. Лаврентьева; см. [3]). В этом случае для модуля непрерывности $\omega(f, \bar{D}, \delta)$ функции $f(z)$ на $\bar{D}$ в [3] получено неравенство $\omega(f, \bar{D}, \delta) \leqslant B(f) \delta^{\alpha}$, где $B(f)>0$ не зависит от $\delta \geqslant 0$, а $\alpha=2 /(c+1)^{2}$, $0<\alpha<1 / 2$.

В приводимых ниже теоремах качество произвольной жордановой кривой $L$ характеризуется ее модулем колебания $d(L ; \delta)$, а в случае спрямляемости $L-$ также и более узкой и чувствительной характеристикой - ее модулем спрямляемости $m(L ; \delta)$ (см. п. 1.1). Эти понятия введены автором в работах [4], [5] (см. также [6]-[8]). В приводимых ниже теоремах "гладкость" отображающих функций характеризуется их обычными модулями непрерывности. Результаты настоящей работы всегда содержательны, они существенно обобщают и усиливают теоремы работ [4]-[7]. Анонсированы они в [8].

\section{§1. Определения, обозначения}

1.1. Модуль колебания и модуль спрямляемости. Модулем колебания $d(L ; \delta)$ жордановой кривой $L$ (замкнутой или разомкнутой; в случае разомкнутой кривой $L$, т.е. дуги, она может содержать или не содержать свои концы) назовем функцию

$$
d(L ; \delta):=\sup \{d(L ; z, t): z, t \in L,|z-t| \leqslant \delta\}, \quad \delta \geqslant 0,
$$

где $d(L ; z, t)$ в случае разомкнутой кривой $L$ обозначает диаметр дуги кривой $L$ с концами $z$ и $t$ и $d(L ; z, t)$ в случае замкнутой кривой $L$ обозначает меньший (точнее, не больший) из диаметров двух дуг кривой $L$ с концами $z$ и $t$. Очевидно, $d(L ; \delta) \leqslant \operatorname{diam}(L)$, где $\operatorname{diam}(L):=\sup \{|z-t|: z, t \in L\}-$ диаметр кривой $L$.

Для спрямляемых жордановых кривых $L$ в [4] введена еще одна характеристика их "качества" - модуль спрямляемости $m(L ; \delta)$ :

$$
m(L ; \delta):=\sup \{m(L ; z, t): z, t \in L,|z-t| \leqslant \delta\}, \quad \delta \geqslant 0,
$$

где $m(L ; z, t)$ в случае разомкнутой кривой $L$ обозначает длину дуги $L(z, t) \subset L$ с концами $z, t$ и $m(L ; z, t)$ в случае замкнутой кривой $L$ обозначает меньшую (точнее, не большую) из длин двух дуг кривой $L$ с концами $z, t$. Для общности записи будем считать $m(L ; \operatorname{diam}(L))=+\infty$ в случае ограниченной неспрямляемой жордановой кривой $L$.

Некоторые свойства модуля колебания и модуля спрямляемости.

1) Если $L$ - открытая жорданова дуга, то $d(\bar{L} ; \delta)=d(L ; \delta), \delta \geqslant 0$, а если при этом $L$ спрямляема $(|L|<\infty)$, то и $m(\bar{L} ; \delta)=m(L ; \delta), \delta \geqslant 0$.

$2)$ При любой жордановой кривой $L$ (разомкнутой или замкнутой) функции $d(L ; \delta)$ (и при дополнительном условии $|L|<\infty$ - функция $m(L ; \delta))$ не убывают и ограничены на $[0,+\infty)$, поэтому они непрерывны в каждой точке $\delta \geqslant 0$, кроме разве лишь конечного или счетного их числа, а в точках разрыва эти функции имеют положительные скачки. 
3 ) Для любой (разомкнутой или замкнутой) жордановой кривой $L$ выполнены соотношения:

$$
\begin{gathered}
d(L ; \delta) \leqslant d(L ; \operatorname{diam}(L))=\operatorname{diam}(L), \quad \delta \geqslant 0, \\
d(L ; \delta) \equiv \operatorname{diam}(L), \quad \delta \geqslant \operatorname{diam}(L) .
\end{gathered}
$$

Верны также следующие соотношения: $m(L ; \delta) \leqslant m(L ; \operatorname{diam}(L))=|L|, \delta \geqslant 0$, если кривая $L$ разомкнута; $m(L ; \delta) \leqslant m(L ; \operatorname{diam}(L))=|L| / 2, \delta \geqslant 0$, если кривая $L$ замкнута; $m(L ; \delta) \geqslant d(L ; \delta), \delta \geqslant 0, m(L ; \delta) \geqslant \delta, \delta \in[0, \operatorname{diam}(L)]$.

4) При $\delta \rightarrow 0$ имеем $d(L ; \delta) \rightarrow 0$ для любой жордановой кривой $L$, а если при этом $L$ спрямляема, то и $m(L ; \delta) \rightarrow 0$; в частности, в этих случаях $d(L ; 0+)=$ $d(L ; 0)=0$ и $m(L ; 0+)=m(L ; 0)=0$ соответственно.

$5)$ Если кривая $L$ жорданова (разомкнутая, т.е. дуга, или замкнутая), то функция $d(L ; \delta)$ непрерывна справа в каждой точке $\delta \geqslant 0$, т.е. $\lim _{\delta^{\prime} \searrow \delta} d\left(L ; \delta^{\prime}\right)=$ $d(L ; \delta)$, а если при этом $|L|<\infty$, то в каждой точке $\delta \geqslant 0$ непрерывна справа и функция $m(L ; \delta)$.

6) Пусть $L$ - гладкая жорданова кривая (т.е. имеющая непрерывно изменяющийся угол положительно направленной касательной с положительным направлением действительной оси при непрерывном движении точки касания по $L$ в положительном направлении). Тогда:

a) существует такое число $\delta(L)>0$, что $d(L ; \delta) \equiv \delta$ при $\delta \in[0, \delta(L)]$;

b) $m(L ; \delta) \geqslant \delta, \delta \in[0, \operatorname{diam}(L)]$, и для любого числа $c>1$ существует такое число $\delta(L, c)>0$, что $m(L ; \delta) \leqslant c \delta, \delta \in[0, \delta(L, c)]$.

$7)$ В случае конечности длины $|L|$ жордановой кривой $L$ характеристика $m(L ; \delta)$ является существенно более информативной, чем $d(L ; \delta)$. Например, если о жордановой кривой $L$ с концами нуль и три известно лишь то, что $m\left(L ; \delta^{\prime}\right)=\delta^{\prime}$ при некотором $\delta^{\prime}>0$, то $L=[0,3]$. В то же время, к примеру, для кривой $L$, составленной из отрезков $[0,1]$ и $[2,3]$, соединенных полуокружностью с диаметром $[1,2]$, имеем $d(L ; \delta)=\delta$ при любом $\delta \in[0,3]$.

1.2. Классы $J(g)$ и $J_{0}(g), J(g, \varepsilon)$ и $J_{0}(g, \varepsilon)$ жордановых кривых. Пусть функция $g(x)$ определена и не убывает при $x \geqslant 0$ (она может иметь положительные разрывы-скачки), $g(x) \geqslant x, x \geqslant 0, g(0)=g(0+)=0$. Через $J(g)$ и $J(g, \varepsilon)(\varepsilon=$ const $>0)$ обозначим семейства всех жордановых кривых $L$ (замкнутых и разомкнутых, с концами и без них), для которых выполнение неравенства $d(L ; \delta) \leqslant g(\delta)$ (см. (1.1)) требуется, соответственно, при всех $\delta \geqslant 0$, или же лишь при $\delta \in[0, \varepsilon]$. Аналогично, $L \in J_{0}(g)$ либо $L \in J_{0}(g, \varepsilon)$ в зависимости от того, выполнение неравенства $m(L ; \delta) \leqslant g(\delta)$ (см. $(1.2))$ требуется для всех $\delta \geqslant 0$ или же лишь для $\delta \in[0, \varepsilon]$. Очевидно, что каждый из этих четырех классов кривых не сузится, если $g(x)$ заменить какой-либо функцией $g_{1}(x) \geqslant g(x), x \geqslant 0$, с теми же общими свойствами, что у $g(x)$. Очевидно также, что $J_{0}(g) \subset J(g), J_{0}(g, \varepsilon) \subset J(g, \varepsilon)$, что с ростом $\varepsilon$ классы $J(g, \varepsilon)$ и $J_{0}(g, \varepsilon)$ не расширяются и что $J(g)=\bigcap_{\varepsilon>0} J(g, \varepsilon), J_{0}(g)=\bigcap_{\varepsilon>0} J_{0}(g, \varepsilon)$.

Важно отметить, что каждая жорданова кривая $L$ принадлежит некоторому классу $J(g)$ и всем классам $J(g, \varepsilon)$ с этим $g$. В качестве $g(x)$ можно взять, например, функцию

$$
\begin{aligned}
& g(x) \equiv d(L ; x) \\
& x \in[0, \operatorname{diam}(L)], \\
& g(x) \equiv d(L ; \operatorname{diam}(L))+(x-d(L ; \operatorname{diam}(L)))^{+}, \quad x \geqslant \operatorname{diam}(L)
\end{aligned}
$$


(здесь, как обычно, $a^{+}:=0$ при $a \leqslant 0, a^{+}:=a$ при $a \geqslant 0$ ). Все требуемые свойства этой функции $g(x)$ следуют из вышеупомянутых свойств 2)-5) модулей колебания и спрямляемости кривой $L$ : например, неравенство $g(x) \geqslant x$ при $x \in[0, \operatorname{diam}(L)]$ вытекает из свойства 3) непосредственно, а при $x \geqslant \operatorname{diam}(L)-$ из свойства 3) и равенств: $g(x) \equiv d(L ; \operatorname{diam}(L))$ при $x \in[\operatorname{diam}(L), d(L ; \operatorname{diam}(L))]$, $g(x) \equiv x$ при $x \geqslant \operatorname{diam}(L)$ (см. выше определение $g(x))$.

Аналогично, каждая спрямляемая жорданова кривая $L$ принадлежит некоторому классу $J_{0}(g)$ и всем классам $J(g, \varepsilon)$ с этим $g$ (например, с $g(x) \equiv m(L ; x)$ при $x \in[0, \operatorname{diam}(L)], g(x) \equiv m(L ; \operatorname{diam}(L))+(x-m(L ; \operatorname{diam}(L)))^{+}$при $x \geqslant$ $\operatorname{diam}(L))$.

В $\S \S 3-6$ определяются достаточно несложные нелинейные операторы, которые для любого однолистного конформного отображения $w=\psi(z)$ произвольной односвязной ограниченной области $G$ с жордановой (например) границей $\partial G$ из какого-либо класса $J(g), \ldots, J_{0}(g, \varepsilon)$ позволяют по функции $g$ найти всегда содержательную оценку для модуля непрерывности $\omega(\psi, \bar{G}, \delta)$ отображения $\psi$ на замыкании области $G$. На самом деле для получения такого типа оценок не обязательно знать значения $g(x)$ для всех $x \geqslant 0$, достаточно их знать хотя бы для $x \in[0, \varepsilon]$ при каком-либо числе $\varepsilon>0$ (см. $\S 3-6)$. Поэтому естественно ввести классы $J(g, \varepsilon)$ и $J_{0}(g, \varepsilon)$ жордановых кривых $L$ требованием выполнения неравенства $d(L ; \delta) \leqslant g(\delta)$ или $m(L ; \delta) \leqslant g(\delta)$ уже лишь для $\delta \in[0, \varepsilon]$ и функции $g(x)$, определенной и не убывающей на $[0, \varepsilon]$ и такой, что $g(x) \geqslant x, x \in[0, \varepsilon], g(0+)=g(0)=0$. Если бы при этом нашлось такое продолжение $g^{*}(x)$ функции $g(x)$ с $[0, \varepsilon]$ на $[0,+\infty)$ (удовлетворяющее уже на $[0,+\infty)$ всем только что перечисленным условиям для $g(x))$, что $g^{*}(x)=g(x), x \in[0, \varepsilon]$, то нововведенные классы $J(g, \varepsilon)$ и $J_{0}(g, \varepsilon)(g(x)$ определена на $[0, \varepsilon])$ совпали бы с ранее введенными классами $J(g, \varepsilon)$ и $J_{0}(g, \varepsilon)$ при $g=g^{*}$ соответственно. Так как при использовании ранее введенных классов $J(g, \varepsilon)$ и $J_{0}(g, \varepsilon)$ (с функцией $g(x)$, удовлетворяющей всем нужным условиям при $x \geqslant 0)$ приводит к более удобным и красивым неравенствам для модулей непрерывности функций $w=\varphi(z)$, конформно отображающих на круг $D$ ограниченные области $G$ с жордановыми границами $\partial G$ из классов $J(g, \varepsilon)$ и $J_{0}(g, \varepsilon)$ (или с более общими границами), а также для обратных к ним функций $z=\psi(w)$, то в дальнейшем мы будем рассматривать эти классы лишь в их первоначальном определении. Но, стремясь получить результаты максимальной общности, нужно предварительно решить указанную выше задачу о продолжении функции $g(x)$ с $[0, \varepsilon]$ на $[0,+\infty)$. Конечно, имеется бесконечно много таких продолжений. Вот примеры таких продолжений: первое, (1.3), связано с классами $J(g, \varepsilon)$ и $J\left(g_{d}^{*}\right)$; второе, $(1.4)$, связано с $J_{0}(g, \varepsilon)$ и $J_{0}\left(g_{m}^{*}\right)$ (см. выше конец предыдущего абзаца и свойство 3)):

$$
\begin{aligned}
g^{*}(x) & :=\min \{g(x), \operatorname{diam}(L)\}, & & x \in[0, \varepsilon], \\
g^{*}(x) & :=\operatorname{diam}(L)+(x-\operatorname{diam}(L))^{+}, & & x>\varepsilon, \\
g^{*}(x) & :=\min \{g(x),|L|\}, & & x \in[0, \varepsilon], \\
g^{*}(x) & :=|L|+(x-|L|)^{+}, & & x>\varepsilon .
\end{aligned}
$$

Заметим, что в случае замкнутой спрямляемой кривой $L$ можно в последнем равенстве взять $|L| / 2$ вместо $|L|$; см. выше 3$)$. 
Очевидно, что классы $J\left(g^{*}, \varepsilon\right)$ и $J_{0}\left(g^{*}, \varepsilon\right)$, если их понимать по-старому и с заменой $g$ на $g^{*}$, не зависят от выбора продолжения $g^{*}$ функции $g$ с отрезка $[0, \varepsilon]$ на интервал $(0,+\infty)$; при этом $J\left(g^{*}, \varepsilon\right)=J(g, \varepsilon)$ и $J_{0}\left(g^{*}, \varepsilon\right)=J_{0}(g, \varepsilon)$, если $J(g, \varepsilon)$ и $J_{0}(g, \varepsilon)$ понимать по-новому - как классы жордановых кривых, определяемые по заданной на $[0, \varepsilon]$ функции $g(x)$. Разумеется, при получении оценок указанного типа для модулей непрерывности функций $\varphi$ и $\psi$ нужно помнить, что неравенства $d(L ; \delta) \leqslant g^{*}(\delta)$ и $m(L ; \delta) \leqslant g^{*}(\delta)$, определяющие классы $J\left(g^{*}\right)$ и $J_{0}\left(g^{*}\right)$ кривых $L$ и выполняющиеся при $\delta \in[0, \varepsilon]$, уже, вообще говоря, не выполняются при всех $\delta \geqslant 0$. Однако для функций $g_{d}^{*}(x)$ и $g_{m}^{*}(x)$, определенных в $(1.3)$ и $(1.4)$, неравенства $d(L ; \delta) \leqslant g_{d}^{*}(\delta)$ и $m(L ; \delta) \leqslant g_{m}^{*}(\delta)$ выполняются при всех $\delta \geqslant 0$, так что $J(g, \varepsilon) \subset J\left(g_{d}^{*}\right)$ и $J_{0}(g, \varepsilon) \subset J\left(g_{m}^{*}\right)$. Здесь число $\varepsilon>0$ - любое фиксированное, а классы $J(g, \varepsilon)$ и $J_{0}(g, \varepsilon)$ жордановых кривых $L$ определяются по функции $g(x)$, заданной на отрезке $[0, \varepsilon]$ и обладающей на нем указанными выше свойствами.

Следует пояснить, зачем вместе с классами $J(g)$ и $J_{0}(g)$ выше введены более широкие классы $J(g, \varepsilon)$ и $J_{0}(g, \varepsilon)$. Дело в том, что иногда достижимая жорданова граничная дуга области $G$ (в частности, вся ее граница $\Gamma \in J(g)$ ) принадлежит еще и некоторому классу $J(q, \varepsilon)$ с функцией $q$, в определенном смысле лучшей, чем $g$. А это позволяет получить более сильную оценку порядка малости (при $\delta \rightarrow 0$ ) модуля непрерывности на дуге $\gamma=\psi^{-1}(\Gamma)$ (и на $\bar{D}$ при $\Gamma=\partial G)$ любого однолистного конформного отображения $w=\psi(z)$ круга $D$ на область $G$, а также модуля непрерывности обратного отображения $w=\varphi(z)=\psi^{-1}(z)$ области $G$ на круг $D($ см. $\S \S 3-6)$. Это видно на следующем примере.

Для любого сколь угодно большого числа $c_{0}>1$ легко построить такие замкнутые жордановы кривые Г (например, сильно сплющенные по вертикали эллипсы), что: I) $\Gamma \in J\left(g_{0}\right)$ при $g_{0}(x):=c_{0} x$, но $\Gamma \notin J(g)$ при $g(x):=c x$, каково бы ни было $c<c_{0}$; II) для каждого числа $c>1$, сколь угодно близкого к единице, найдется такое число $\varepsilon(c)>0$, что $\Gamma \in J_{0}(g, \varepsilon(c))$. Приводимые ниже теоремы 2.1 и 2.2 показывают, насколько существенно различны заключения о "качестве" модуля непрерывности конформного отображения $w=\psi(z)$ круга $D$ на внутренность $G$ такой кривой Г при условиях типа I) и типа II).

В связи со сказанным сделаем еще одно замечание: если $L$ - замкнутая спрямляемая жорданова кривая и при некотором постоянном $c>1$ верно неравенство $m(L ; \delta) \leqslant c \delta$ для любого $\delta \geqslant 0$, то $c \geqslant \pi / 2$, а равенство $c=\pi / 2$ возможно лишь в случае, если $L-$ окружность. Это же означает, что зависящие от $c$ оценки для модулей непрерывности конформных отображений круга $D$ на области с жордановыми спрямляемыми границами класса $J_{0}(g)$ при $g(x) \leqslant c x$ могут работать лишь при $c \geqslant \pi / 2$. Введение свободных от такого недостатка классов $J(g, \varepsilon)$ и $J_{0}(g, \varepsilon)$ вызвано, в частности, и этим тоже.

1.3. Достижимые точки и достижимые дуги границы области. Напомним два определения из теории простых концов Каратеодори.

Пусть в точку $\zeta$ границы $\partial G$ некоторой односвязной области $G$ комплексной плоскости $\mathbb{C}$ ведет изнутри $G$ жорданов путь (т.е. направленная жорданова дуга) $L(\zeta) \subset G \cup\{\zeta\}$ с концом в точке $\zeta$ и остальными своими точками лежащий в $G$; два жордановых пути $L^{\prime}(\zeta)$ и $L^{\prime \prime}(\zeta)$ с теми же свойствами, что и $L(\zeta)$, называют эквивалентными или, что то же самое, определяющими одну и ту 
же достижимую граничную точку области $G$ с носителем $\zeta$, если существует третий путь $L(\zeta)$ с теми же свойствами, пересекающийся и с $L^{\prime}(\zeta)$, и с $L^{\prime \prime}(\zeta)$ внутри $G$ в любой окрестности точки $\zeta$. Говоря несколько иными словами, достижимая граничная точка области $G$ с носителем $\zeta \in \partial G$ определяется как класс всех таких попарно эквивалентных путей с концами в точке $\zeta$. Несложные примеры показывают, что различные достижимые точки границы области могут иметь один и тот же носитель (т.е. геометрически изображаться одной и той же точкой границы) и что у некоторых областей имеются недостижимые точки их границ. И еще одно важное (хотя и простое) утверждение: если граница односвязной области $G$ является замкнутой жордановой кривой, то каждая точка $\zeta \in \partial G$ является достижимой граничной точкой этой области, причем сама точка $\zeta$ является носителем единственной достижимой граничной точки (т.е. различные точки на $\partial G$ являются носителями различных достижимых граничных точек этой области).

Жорданова кривая Г - разомкнутая (иначе говоря, дуга с концами или без них, т.е. дуга замкнутая или открытая соответственно) или замкнутая кривая (т.е. контур) на границе области $G$ называется достижимой из $G$, если найдется такая односвязная область $\Omega=\Omega(G, \Gamma) \subset G$ с жордановой границей $\partial \Omega$, что $\partial \Omega \cap \partial G=\bar{\Gamma} ;$ при этом $\bar{\Gamma}$ называется носителем достижимой граничной дуги $\Gamma$.

Очевидно, каждая точка замыкания достижимой жордановой дуги Г границы области $G$ является достижимой из $\Omega(G, \Gamma)$ и из $G$ точкой.

Для упрощения формулировок некоторых теорем в случае жордановой области $G$ (т.е. в случае, когда $\partial G$ - жорданов контур) будем называть $\partial G$ открытой достижимой жордановой дугой границы области $G$ (об этом уже говорилось выше); очевидно, что в этом случае $\Omega(G, \partial G)=G$. Из определений видно, что замыкание открытой достижимой жордановой дуги является замкнутой достижимой жордановой дугой, так что концы открытой достижимой жордановой дуги $\Gamma$ при условии $\bar{\Gamma} \neq \partial G$ не совпадают между собой, и при этом условии $\Omega \neq G$, а жорданова дуга $G \cap \partial \Omega$ является (жордановым) разрезом односвязной области $G$ на две непересекающиеся односвязные ее подобласти (жордановы при жордановости $G$ ).

Заметим, что одна жорданова дуга $\Gamma \neq \partial G$ может быть носителем двух непересекающихся достижимых дуг границы некоторой односвязной области.

1.4. Некоторые обозначения. Пусть $G$ - односвязная область комплексной плоскости $\mathbb{C}$ с границей $\partial G, \Gamma$ - ограниченная открытая достижимая жорданова дуга ее границы, $w=\varphi(z)$ - некоторое однолистное конформное отображение этой области на единичный круг $D, w=\psi(z)$ - обратное однолистное конформное отображение единичного круга $D$ на эту область $\left(\psi(z) \equiv \varphi^{-1}(z)\right.$, $z \in D), \gamma:=\varphi(\Gamma) \subset C:=\partial D$. Если открытая достижимая жорданова дуга $\Sigma \subset \Gamma$ лежит в $\Gamma$ вместе со своим замыканием $\bar{\Sigma}$, то дуга $\sigma:=\varphi(\Sigma)$ лежит в $\gamma$ вместе со своим замыканием $\bar{\sigma}$.

Введем также следующие обозначения:

$$
\begin{gathered}
\sigma_{t}(r):=\{z:|z|=1,|z-t| \leqslant r\}, \quad t \in C, \quad 0<r<2, \quad \Sigma_{t}(r):=\psi\left(\sigma_{t}(r)\right), \\
\lambda_{t}(r):=\{z:|z-t|=r,|z| \leqslant 1\}, \quad \Lambda_{t}(r):=\psi\left(\lambda_{t}(r)\right), \quad l_{t}(r):=\left|\Lambda_{t}(r)\right|, \\
\Delta_{t}(r):=\{z:|z|<1,|z-t|<r\}, \quad S_{t}(r):=\operatorname{mes}_{2}\left(\psi\left(\Delta_{t}(r)\right)\right) .
\end{gathered}
$$


Если $\partial G$ - замкнутая жорданова кривая, то

$$
\begin{gathered}
d_{t}(\psi):=\max \left\{r: \operatorname{diam}\left(\Sigma_{t}(r)\right), \leqslant \operatorname{diam}\left(\partial G \backslash \Sigma_{t}(r)\right)\right\}, \\
d(\psi):=\min \left\{d_{t}(\psi): t \in C\right\} .
\end{gathered}
$$

Если $\partial G$ - замкнутая спрямляемая жорданова кривая, то

$$
\begin{gathered}
m_{t}(\psi):=\max \left\{r:\left|\Sigma_{t}(r)\right| \leqslant\left|\Gamma \backslash \Sigma_{t}(r)\right|\right\}, \\
m(\psi):=\min \left\{m_{t}(\psi): t \in C\right\} .
\end{gathered}
$$

Для какого-либо числа $\varepsilon>0$ и открытой достижимой жордановой дуги $\Gamma$ границы области $G$ при $\Gamma \neq \partial G$ обозначим через $\bar{\rho}=\bar{\rho}(\psi, \Gamma, \varepsilon)$ супремум всех чисел $r>0$, для каждого из которых верно неравенство $\operatorname{diam}\left(\Sigma_{t}(r)\right) \leqslant \varepsilon$ при всех таких $t \in \gamma=\psi^{-1}(\Gamma)$, что $\sigma_{t}(r) \subset \gamma$ (или, что то же, $\left.\Sigma_{t}(r) \subset \Gamma\right)$. Если же $\Gamma=\partial G$, то в приведенном определении $\bar{\rho}(\psi, \Gamma, \varepsilon)$ нужно заменить условие $r>0$ условием $r \in(0, d(\psi)]$ (определение $d(\psi)$ см. выше).

Для ограниченной открытой достижимой жордановой дуги Г границы односвязной области $G \subset \mathbb{C}$, открытой достижимой жордановой дуги $\Sigma \subset \bar{\Sigma} \subset \Gamma$, конформного отображения $w=\psi(z)$ круга $D$ на область $G$, некоторой точки $t \in \gamma:=\psi^{-1}(\Gamma)$ и числа $\varepsilon>0$ положим при $\Gamma \neq \partial G$

$$
\begin{gathered}
\bar{\rho}(\psi, \Gamma, t, \varepsilon):=\sup \left\{r: r \in(0, \rho(t, C \backslash \gamma)), \operatorname{diam}\left(\Sigma_{t}(r)\right) \leqslant \varepsilon\right\}, \\
\bar{\rho}(\psi, \Gamma, \Sigma, \varepsilon):=\inf \{\bar{\rho}(\psi, \Gamma, t, \varepsilon): t \in \bar{\sigma}\},
\end{gathered}
$$

где $\rho(E, F)$ - расстояние между множествами $E$ и $F$. Если $\Gamma=\partial G$, то в этом определении нужно условие $r>0$ заменить условием $r \in(0, d(\psi)]$ (определение $d(\psi)$ см. выше).

Если дополнительно к сказанному о $G, \Gamma$ и $\Sigma$ известно, что дуга $\Gamma$ имеет конечную длину $|\Gamma|$, то совершенно аналогично предыдущему определяем величины $\bar{\rho}_{0}(\psi, \Gamma, t, \varepsilon)$ и $\bar{\rho}_{0}(\psi, \Gamma, \Sigma, \varepsilon)$ : нужно в определениях величин $\bar{\rho}(\cdot, \cdot, \cdot, \cdot)$ заменить диаметры дуг $\operatorname{diam}\left(\Sigma_{t}(r)\right)$ длинами этих дуг $\left|\Sigma_{t}(r)\right|$, а ссылку величины $d(\psi)$ заменить ссылкой на $m(\psi)$ (см. выше).

Так как вдоль дуги $\bar{\Gamma}$ функция $\psi(z)$ равномерно непрерывна (см. п. 2.2) и ни на какой открытой дуге $\Gamma_{1} \subset \Gamma$ она не постоянна (по граничной теореме единственности для аналитических функций), то все величины $\bar{\rho}(\psi, \Gamma, t, \varepsilon)$, $\bar{\rho}(\psi, \Gamma, \Sigma, \varepsilon), \bar{\rho}_{0}(\psi, \Gamma, t, \varepsilon)$ и $\bar{\rho}_{0}(\psi, \Gamma, \Sigma, \varepsilon)$ всегда положительны.

1.5. Функции-интегралы $F(r)$ и $U(a ; x)$. Введем еще два обозначения.

$$
F(r)=F(\psi, t ; r):=\int_{0}^{r} l_{t}^{2}(v) \frac{d v}{v} \quad(\leqslant+\infty), \quad r \geqslant 0 .
$$

Отметим, что при $v>2$ и любом $t \in C$ множество $\Lambda_{t}(v)$ пусто, $l_{t}(v)=0$, так что $F(r) \equiv F(2)$ при $r \geqslant 2$.

Если функция $y=u(x)$ определена и строго возрастает при $x \geqslant 0$ (она может быть разрывной), $u(0)=u(0+)=0, u(x) \geqslant x$, то обратная к $u(x)$ функция $x=$ $u^{-1}(y), y \geqslant 0$, непрерывна (разрыву-скачку величины $h=h\left(x_{0}\right)>0$ функции $y=u(x)$ в некоторой точке $x_{0}>0$ соответствует отрезок длины $h$ постоянства функции $\left.x=u^{-1}(y)\right)$. Фиксируем некоторое число $a>0$ и положим (см. [4], [5])

$$
U(a ; x)=U(u, a ; x):=\int_{a}^{x} \frac{d y}{u^{-1}(y)}, \quad U(x):=U(u, 1 ; x), \quad x>0 .
$$


ЗАмечАниЕ 1.1. Нетрудно видеть, что на интервале $(0,+\infty)$ функция $y=$ $U(a ; x)$ определена, непрерывна, выпукла вверх и строго возрастает от $-\infty=$ : $U(a ; 0)$ до $+\infty=: U(a ;+\infty)$ (интеграл (1.6) расходится к $+\infty$ при $x=+\infty$, так как $\left.0<u^{-1}(y)<y\right)$. Обратная же к $U(a ; x)$ функция $U^{-1}(a ; y)$ определена, непрерывна, положительна и выпукла вниз на $(-\infty,+\infty)$, строго возрастает на $[-\infty,+\infty]$ от $0=: U^{-1}(a ;-\infty)$ до $+\infty=: U^{-1}(a ;+\infty)$ (ее график похож на график функции $\left.x=a e^{y}\right), U^{-1}(a ; 0)=a$. Отметим также, что если функция $u_{1}(x)$ обладает всеми перечисленными выше свойствами функции $u(x)$ и совпадает с ней на некотором отрезке вида $[0, \xi], \xi=$ const $>0$, то $u_{1}^{-1}(y) \equiv u^{-1}(y)$ при $y \in[0, u(\xi)], U_{1}(a ; x) \equiv U(a ; x)$ при $x \in(0, u(\xi)]\left(U_{1}(a ; x):=U\left(u_{1}, a ; x\right)\right)$, $U_{1}^{-1}(a ; y) \equiv U^{-1}(a ; y)$ при $y \in[-\infty, U(a ; u(\xi))]$. Нетрудно также проверить справедливость более общего утверждения: если $u_{1}(x)$ обладает всеми перечисленными выше свойствами функции $u(x)$ и $u_{1}(x) \leqslant u(x)$ для всех $x \in[0, \xi]$ при $0<\xi=\mathrm{const} \leqslant a$, то $U_{1}^{-1}(a ; y) \leqslant U^{-1}(a ; y)$ при всех $y \in[-\infty, U(a ; u(\xi))]$.

\section{§ 2. Вспомогательные результаты}

2.1. Некоторые свойства модуля непрерывности. Для удобства ссылок отметим здесь некоторые простые свойства модуля непрерывности. Именно, если функция $f(z)$ непрерывна на компакте $E$, то при любых неотрицательных $\delta$ и $\eta$ ее модуль непрерывности на $E$ удовлетворяет условиям:

$$
0=\omega(f, E, 0)=\omega(f, E, 0+) \leqslant \omega(f, E, \delta) \leqslant \omega(f, E, \delta+\eta) .
$$

Если $f(z)$ определена и ограничена на некотором выпуклом множестве $E$, то

$$
\omega(f, E, \delta) \leqslant \omega(f, E, \delta+\eta) \leqslant \omega(f, E, \delta)+\omega(f, E, \eta)
$$

(так называемое свойство полуаддитивности или квазивыпуклости). Из него вытекают еще два свойства модулей непрерывности функций $f$, ограниченных на выпуклых множествах $E: \omega(f, E, n \delta) \leqslant n \omega(f, E, \delta), \delta \geqslant 0, n \in \mathbb{N}$, и

$$
\omega(f, E, \lambda \delta) \leqslant(\lambda+1) \omega(f, E, \delta), \quad \delta \geqslant 0, \quad \lambda>0 .
$$

\section{2. Локальная форма теоремы Каратеодори.}

Теорема 2.1. Пусть Г - ограниченная открытая достижимая жорданова дуга границы (см. п. 1.3) односвязной области $G \subset \mathbb{C}$ (не обязательно жсордановой или ограниченной), a $w=\varphi(z)$ - некоторое однолистное конформное отображение этой области на единичный круг $D$. Тогда это отображение продолжается с $G$ на $\bar{\Gamma}$ до гомеоморфизма множества $G \cup \Gamma$ на множество $D \cup \gamma$, где $\gamma:=\varphi(\Gamma)$, а также гомеоморфизма замкнутой дуги $\bar{\Gamma}$ на замкнутую дугy $\bar{\gamma}$.

Доказательство этого утверждения проводится теми же методами, что и доказательство обычной теоремы Каратеодори (например, см. [9; гл. 2, §3]).

2.3. Теорема М. А. Лаврентьева. Ниже нам понадобится один частный случай теоремы М. А. Лаврентьева из работы [10]. Прежде чем сформулировать это утверждение, напомним некоторые определения из этой работы. 
Пусть $G$ - ограниченная односвязная область плоскости $\mathbb{C}, 0 \in G$, и фиксированы две точки $z$ и $t$ внутри $G$. Обозначим через $\rho_{1}(G ; z, t)$ инфимум длин всех спрямляемых кривых с концами $z$ и $t$, всеми своими точками лежащих в $G, 0 \leqslant \rho_{1}(G ; z, t)<+\infty$. Через $\rho_{2}(G ; z, t)$ обозначим инфимум длин всех спрямляемых жордановых разрезов области $G$, разбивающих ее на две непересекающиеся односвязные области, одна из которых (обозначим ее через $G(0)$ ) содержит точку нуль, другая (обозначим ее через $G(z, t):=G \backslash \overline{G(0)})$ содержит точки $z$ и $t$ внутри себя. Положим

$$
\rho(G ; z, t):=\min \left\{\rho_{1}(G ; z, t), \rho_{2}(G ; z, t)\right\} .
$$

Далее, если одна из точек $z, t$ (для определенности пусть это будет $z$ ) является достижимой граничной точкой области $G$ по некоторому жорданову пути $L(z) \subset G \cup\{z\}$ (см. п. 1.3), а $t \in G$, то полагаем

$$
\rho(G ; z, t):=\lim _{L(z) \ni z^{\prime} \rightarrow z} \rho\left(G ; z^{\prime}, t\right) ;
$$

если же точка $z \in \partial G$ достижима из $G$ по кривой $L(z)$, а точка $t \in \partial G$ достижима из $G$ по кривой $L(t)$, то $\rho(G ; z, t)$ определяется как предел величины $\rho\left(G ; z^{\prime}, t^{\prime}\right)$ при стремлении $z^{\prime}$ к $z$, а $t^{\prime}$ к $t$ по соответствующим кривым $L(z)$, $L(t)$. Очевидно, всегда $0 \leqslant \rho(G ; z, t)<+\infty$. Будем называть $\rho(G ; z, t)$ paccmoянием Лаврентъева между точками $z$ и $t$ в области $G$, хотя эта величина не является метрикой в строгом смысле слова: вообще говоря, для нее не выполняется неравенство треугольника. Отметим, что Г. Д. Суворов в [11] устранил этот недостаток, подкорректировав определение расстояния Лаврентьева.

Теорема 2.2 (М. А. Лаврентьев; см. [10]). Пусть $G$ - ограниченная односвязная область, $0 \in G, w=f(z)$ - такое однолистное конформное отображение области $G$ на единичный круг $D$, что $f(0)=0$ u $f^{\prime}(0)=1$. Тогда для любых двух точек z и $t$, каждая из которых является либо внутренней точкой области $G$, либо достижимой точкой ее границы, имеет место неравенство

$$
|f(z)-f(t)| \leqslant K_{\Lambda} \cdot \sqrt{\rho(G ; z, t)},
$$

где $K_{\Lambda}-$ абсолютная постоянная Лаврентьева, $K_{\Lambda}>0$.

Отметим, что $\rho(G ; z, t)$ по порядку малости, вообе говоря, не сравнимо с евклидовым расстоянием между $z$ и $t$ : нетрудно построить ограниченную область $G$ и пары точек $z, t \in G$, при одних путях стремления которых к $\partial G$ будет $\rho(G ; z, t)=o(|z-t|)$, при других - $|z-t|=o(\rho(G ; z, t))$. Это делает "метрику" $\rho(G ; z, t)$, очень интересную для исследования некоторых граничных свойств конформных отображений, мало пригодной для непосредственных оценок обычных модулей непрерывности отображающих функций.

2.4. Теорема П. М. Тамразова. Ниже нам будет полезна следующая теорема, связывающая модуль непрерывности функции $f(z)$, однозначной и аналитической в односвязной ограниченной области $G$ и непрерывной на $\bar{G}$, с ее модулем непрерывности на границе $\partial G$ этой области.

Теорема 2.3 (П. М. Тамразов; см. [12]). Если модулъ непреръвности $\omega(f$, $\bar{G}, \delta)$ функции $f(z)$, аналитической в ограниченной односвлзной области $G$ 
с жордановой границей $\partial G$ и непрерывной на $\bar{G}$, имеет нормальную мажсоранmу (см. [12]), то ее модули непрерьвности на $\partial G$ и на $\bar{G}$ связань следующим двойным неравенством:

$$
\omega(f, \partial G, \delta) \leqslant \omega(f, \bar{G}, \delta) \leqslant 108 \omega(f, \partial G, \delta), \quad \delta \geqslant 0 .
$$

В случае выпуклости ограниченной области $G$ это неравенство верно для любой функции $f(z)$, аналитической в $G$ и непрерывной на $\bar{G}$.

\section{5. Оценки площади жордановой области через некоторые пара-} метры ее границы (общий случай). Приводимые ниже леммы 2.1-2.7 используются в доказательствах теорем $\S 4$ и теоремы 6.2 из $\S 6$. Лемма 2.8 нужна для доказательства неулучшаемости порядка малости правых частей неравенств, утверждаемых этими теоремами.

ЛЕмма 2.1. Пусть граница $\gamma$ ограниченной замкнутой жордановой области $G$ состоит из двух жордановых дуг $\gamma_{1}$ u $\gamma_{2}$ с общими концами а $и$ b, причем их длины $\left|\gamma_{n}\right|:=\operatorname{mes}_{1}\left(\gamma_{n}\right), n=1,2$, не превосходят чисел $l_{n}$, а их диаметры $\operatorname{diam}\left(\gamma_{n}\right)$ - чисел $d_{n}$ соответственно. Тогда верны следуюшие неравенства:

$$
\begin{aligned}
\operatorname{mes}_{2}(G) & \leqslant \frac{1}{2 \pi} l_{1}^{2}+\frac{\pi}{4} d_{2}^{2}, \\
\operatorname{mes}_{2}(G) & \leqslant \frac{1}{4 \pi}\left(l_{1}+l_{2}\right)^{2} .
\end{aligned}
$$

ДоказАтельство. Соединим точки $a$ и $b$ отрезком $j=[a, b]$. При каждом $n=1,2$ обозначим через $G_{n}$ неограниченную связную открытую компоненту дополнения к компакту $Q_{n}=\gamma_{n} \cup j$, а через $K_{n}$ - дополнение к $G_{n}$ соответственно.

Докажем сначала, что $G \subset K_{1} \cup K_{2}$.

Пусть $c \in G \backslash j$. Индекс $\operatorname{ind}_{L} c$ точки $c$ относительно замкнутой кривой $L$ по определению равен деленному на $2 \pi$ приращению $\Delta_{L} \operatorname{Arg}(z-c)$ функции $\operatorname{Arg}(z-c)$ при однократном пробегании точкой $z$ этой кривой в положительном направлении, если при этом значения этой функции изменяются непрерывно. В силу жордановости кривой $\gamma$ имеем равенство

$$
\operatorname{ind}_{\gamma} c=\frac{\Delta_{\gamma_{1}} \operatorname{Arg}(z-c)+\Delta_{\gamma_{2}} \operatorname{Arg}(z-c)}{2 \pi}=1 .
$$

Для определенности предположим, что при обходе $\gamma$ в положительном направлении точка $z$ движется по дуге $\gamma_{1}$ от $a$ к $b$. Тогда по дуге $\gamma_{2}$ она движется от $b$ к $а$. Поэтому

$$
\operatorname{ind}_{\gamma_{1} \cup[b, a]} c+\operatorname{ind}_{\gamma_{2} \cup[a, b]} c=\operatorname{ind}_{\gamma} c=1 \text {. }
$$

Если же $c \notin K_{1} \cup K_{2}$ (т.е. $c \in G_{1} \cap G_{2}$ ), то каждое слагаемое в левой части этого равенства равно нулю. Следовательно, фиксированная ранее точка $c \in$ $G \backslash j$ принадлежит объединению $K_{1} \cup K_{2}$. То, что $\gamma \cup j \subset K_{1} \cup K_{2}$, очевидно. Включение $G \subset K_{1} \cup K_{2}$ доказано.

Докажем неравенство (2.5). Как известно (см. [13; первая часть]), среди всех ограниченных замкнутых множеств $K$, граница $\partial K$ каждого из которых принадлежит объединению некоторой кривой $L(K)$ длины меньше или равной 
$l=$ const и отрезка, соединяющего концы этой кривой, наибольшую площадь, равную $l^{2} /(2 \pi)$, имеет полукруг, ограниченный полуокружностью длины $l$ и ее хордой-диаметром (длины $2 l / \pi$ ). Это так называемая изопериметрическая задача Дидоны. Напомним также, что среди всех измеримых по Лебегу плоских множеств с диаметрами меньше или равными $d$ наибольшую площадь, равную $\pi d^{2} / 4$, имеет круг диаметра $d$ (теорема П. С. Урысона; см. [14; теорема 1]). Так как $\operatorname{mes}_{1}\left(\gamma_{1}\right) \leqslant l_{1}, \operatorname{diam}\left(K_{2}\right)=\operatorname{diam}\left(\gamma_{2}\right) \leqslant d_{2}$, то отсюда получаем неравенство $\operatorname{mes}_{2}(G) \leqslant l_{1}^{2} /(2 \pi)+\pi d_{2}^{2} / 4$.

Докажем неравенство (2.6). Если $\operatorname{mes}_{1}\left(\gamma_{n}\right) \leqslant l_{n}, n=1,2$, то (2.6) следует из того, что среди всех жордановых областей с границами длины меньше или равной $l$ наибольшую площадь, равную $l^{2} /(4 \pi)$, имеет круг радиуса $l /(2 \pi)($ см. [13; первая часть]).

\section{6. Оценки площади жордановой области через некоторые пара-} метры ее границы (частный случай). Целью настоящего пункта является доказательство приводимой ниже в лемме 2.4 оценки площади ограниченной области $G$, границей которой является замкнутая жорданова спрямляемая кривая при некоторых специальных условиях, налагаемых на нее. Формулировке леммы 2.4 предпошлем необходимые определения, а также лемму 2.2 (дающую решение одной экстремальной геометрической задачи) и лемму 2.3 (содержащую некоторые аналитические соотношения).

Обозначения и предварительные результаты. При $x=$ const $\in[0,1]$ и $c=$ const $\in[1,+\infty]$ через $\alpha(x) \in[0,2 \pi]$ и $\beta(c) \in[0,2 \pi]$ обозначим корни следующих уравнений соответственно:

$$
\frac{\sin (\alpha / 2)}{\alpha / 2}=x, \quad \frac{\beta / 2}{\sin (\beta / 2)}=c, \quad \alpha(x) \equiv \beta\left(\frac{1}{x}\right), \quad \beta(c) \equiv \alpha\left(\frac{1}{c}\right) .
$$

Существование и единственность такого $\alpha=\alpha(x)$ при каждом $x \in[0,1]$ и такого $\beta=\beta(c)$ при каждом $c \in[1,+\infty]$ следует из непрерывности и строгого убывания функции $(\sin (y / 2)) /(y / 2)$ на отрезке $[0,2 \pi]$ от единицы до нуля (или, что то же самое, из непрерывности и строгого возрастания функции $(y / 2) / \sin (y / 2)$ от единицы до $+\infty)$ на $[0,2 \pi]$. Здесь и ниже неопределенности вида $0 / 0$ в выражениях типа $(\sin y) / y$ при $y=0$, как и в иных аналогичных случаях, раскрывается переходом в этом выражении к пределу при $y \rightarrow 0$ (точнее, при $y \searrow 0$ ). Поэтому в $(2.7)$ выражение $(\sin (y / 2)) /(y / 2)$ равно единице при $y=0$ и равно нулю при $y=2 \pi$, а выражение $(y / 2) / \sin (y / 2)$ равно единице при $y=0$ и равно $+\infty$ при $y=2 \pi$ (по обобщенной непрерывности этой функции в этой точке) соответственно; $\beta(+\infty)=2 \pi$. Что касается тождеств в $(2.7)$, то они очевидны.

При $c \geqslant 1$ положим

$$
B(c):=c^{2} \frac{\beta(c)-\sin \beta(c)}{2 \beta^{2}(c)}, \quad B(1)=0, \quad B(+\infty)=+\infty .
$$

При $\alpha \in[0,2 \pi]$ и $c \geqslant 1$ положим

$$
S(c, \alpha):=\frac{\alpha-\sin \alpha}{2 \alpha^{2}}+B(c)\left(\frac{\sin (\alpha / 2)}{\alpha / 2}\right)^{2}, \quad S(c, 0) \equiv B(c) .
$$

Дадим еще одно определение, родственное определению $S(c, \alpha)$. Именно, пусть $x \in[0,1]$ и $\alpha \in[0,2 \pi]$ связаны первым равенством из $(2.7)$. Так как 
функция $x=x(\alpha)=(\sin (\alpha / 2)) /(\alpha / 2)$ на $[0,2 \pi]$ однозначна, непрерывна и строго убывает от единицы до нуля, $x^{\prime}(\alpha)<0$ на $(0,2 \pi]$, то обратная функция $\alpha=\alpha(x)$ на $[0,1]$ однозначна, непрерывна и строго убывает от $2 \pi$ до нуля, $\alpha^{\prime}(x)<0$ на $[0,1)$. Пусть

$$
\widetilde{S}(c, x):=S(c, \alpha(x))=\frac{\alpha(x)-\sin \alpha(x)}{2 \alpha^{2}(x)}+B(c) x^{2} .
$$

Величину $S(c)$ определим двумя эквивалентными способами:

$$
S(c):=\max \{S(c, \alpha): \alpha \in[0,2 \pi]\} \equiv \max \{\widetilde{S}(c, x): x \in[0,1]\} .
$$

На полуполосе $\{(c, \alpha): 1 \leqslant c<+\infty, 0 \leqslant \alpha \leqslant 2 \pi\}$ функция $S(c, \alpha)$ непрерывна (по совокупности переменных) и при каждом фиксированном $\alpha \in[0,2 \pi)$ она непрерывна по $c \geqslant 1$ и строго возрастает от $S(1, \alpha)=(\alpha-\sin \alpha) /\left(2 \alpha^{2}\right)$ до $+\infty$, а $S(c, 2 \pi) \equiv 1 /(4 \pi)$. Отсюда заключаем, что функция $S(c)$ непрерывно и монотонно возрастает при $c \geqslant 1$.

Лемма 2.2. Пусть граница $\partial G$ ограниченной области $G$ является замкнутой жордановой спрямляемой кривой, состоящей из двух дуг $L$ и $\Sigma$ с общими кониами $a, b$ и общей хордой $j:=[a, b]$, причем $|L|=l=$ const $>0$, $|j|=|b-a|=x l, 0 \leqslant x \leqslant 1$ (возможно совпадение $a=b$ и тогда $x=0$, a $j$ и $\Sigma$ вырождаются в одну точку), $|\Sigma| \leqslant c|j|$ при некотором $c=$ const $\geqslant 1$ (при $x=1=c$ контур $\partial G$ вырождается в дважды проходимыи отрезок $j$, a $G$ - в пустое множество; при $x=0$ имеем $\partial G=L$, maк что $\operatorname{mes}_{2}(G)$ не превосходит $|L|^{2} /(4 \pi)$ - площади круга с длиной $|L|$ его окружности). Тогда

$$
\operatorname{mes}_{2}(G) \leqslant \widetilde{S}(c, x) l^{2}=S(c, \alpha) l^{2} \leqslant S(c) l^{2} .
$$

ЗАмечАниЕ 2.1. Первое из неравенств (2.12) неулучшаемо: для каждой тройки чисел $l>0, x \in[0,1]$ и $c \geqslant 1$ при условии $x \neq c$ найдутся на плоскости $\mathbb{C}$ такие точки $a, b$ и область $G$, удовлетворяющие вместе с этими $l, x$ и $c$ всем условиям леммы 2.2, что это неравенство обращается в равенство.

Второе из неравенств (2.12) также неулучшаемо: для каждой пары чисел $l>0$ и $c \geqslant 1$ найдутся на плоскости $\mathbb{C}$ такие точки $a, b$ и область $G$, удовлетворяющие вместе с этими $l$ и $c$ всем условиям леммы 2.2, что оба неравенства в (2.12) одновременно обратятся в равенства.

ДокАЗАТЕЛЬство. Будем пользоваться обозначениями леммы 2.1 и первой части ее доказательства.

Оценим величины $S_{n}:=\operatorname{mes}_{2}\left(K_{n}\right), n=1,2$ (см. начало доказательства леммы 2.1). Там, используя решение изопериметрической задачи Дидоны, получено следующее утверждение: $S_{n} \leqslant S_{n}^{*}$, где $S_{n}^{*}$ - площади круговых сегментов $\operatorname{Seg}_{n}$ (пусть они будут замкнутыми) с общей хордой $j$ и с длинами дуг, равными $|L|$ у $\operatorname{Seg}_{1}$ и $c|j|$ у $\operatorname{Seg}_{2}$ соответственно. Будем считать, что эти сегменты лежат на плоскости по разные стороны отрезка $j$ ("как два уха"). Если при этом $|j|=|L|$, то $\operatorname{Seg}_{1}$ вырождается в отрезок $j$ (нулевой площади), а если $c=1$, то в отрезок $j$ вырождается сегмент $\operatorname{Seg}_{2}$. Если же $|j|=0$ (т.е. $a=b$ ), то $\operatorname{Seg}_{2}$ вырождается в точку $a=b$, a Seg $_{1}$ становится замкнутым кругом с длиной границы, равной $|L|$, и площадью $|L|^{2} /(4 \pi)$. 
Очевидно, $\operatorname{mes}_{2}(G) \leqslant S_{1}^{*}+S_{2}^{*}$. Поэтому далее оценим $S_{1}^{*}$ и $S_{2}^{*}$ в невырожденных случаях.

Пусть $R, \alpha$ и $|L|$ - величина радиуса сегмента $\mathrm{Seg}_{1}$, угловая мера его дуги и длина этой дуги соответственно. Величину радиуса сегмента $\mathrm{Seg}_{2}$ и угловую меру его дуги обозначим через $r$ и $\beta$ соответственно. Так как длина дуги сегмента $\operatorname{Seg}_{2}$ равна $c|j|$, то $\beta=c|j| / r$. Так как $|j|=2 r \sin (\beta / 2)$, то $c|j|=c 2 r \sin (\beta / 2), \beta=c|j| / r=2 c \sin (\beta / 2)$. Отсюда $(\beta / 2) / \sin (\beta / 2)=c-$ это второе уравнение в (2.7) для определения $\beta=\beta(c)$ через $c$. Итак, $\beta \in(0,2 \pi)$ в невырожденном случае, т.е. при $c \in(1,+\infty)$. Что касается случая $c=1$ (т.е. случая $\Sigma=j$ ), то считаем (по непрерывности тоже), что $\beta(1)=0$. По обобщенной непрерывности считаем, что $\beta(+\infty)=2 \pi$. Таким образом, $\beta$ зависит лишь от $c \in[1,+\infty]$ и при этом $\beta=\beta(c)$ зависит лишь от $c \in[1,+\infty]$.

Так как площадь треугольника с двумя равными сторонами длины $R$ и углом величины $\nu \in[0, \pi]$ между ними равна $(1 / 2) R^{2} \sin \nu$, а $R=|L| / \nu$, то

$$
S_{1}^{*}=\alpha \frac{1}{2} R^{2}-\frac{1}{2} R^{2} \sin \alpha=\frac{\alpha-\sin \alpha}{2 \alpha^{2}}|L|^{2} .
$$

Аналогично, $S_{2}^{*}=(\beta-\sin \beta) /\left(2 \beta^{2}\right) \cdot(c|j|)^{2}$. Так как $|j|=2 R \sin (\alpha / 2)$, то

$$
\begin{gathered}
c|j|=c 2 \frac{|L|}{\alpha} \sin \frac{\alpha}{2}=c\left\{\frac{\sin (\alpha / 2)}{\alpha / 2}\right\}|L|, \\
S_{2}^{*}=B(c) \frac{\sin (\alpha / 2)}{(\alpha / 2)^{2}}|L|^{2}=B(c) x^{2}|L|^{2} \quad(\text { см. }(2.8) \text { и }(2.7)), \\
\operatorname{mes}_{2}(G) \leqslant S_{1}^{*}+S_{2}^{*}=\left\{\frac{\alpha-\sin \alpha+B(c)(1-\cos \alpha)}{2 \alpha^{2}}\right\}|L|^{2}, \\
\operatorname{mes}_{2}(G) \leqslant S(c, \alpha)|L|^{2}=\widetilde{S}(c, x)|L|^{2} \leqslant S(c) l^{2} .
\end{gathered}
$$

Соотношения (2.12) доказаны.

Справедливость замечания 2.1 следует непосредственно из приведенного выше доказательства леммы 2.2, сводящегося к построению соответствующей экстремальной области $G$.

ЛЕмма 2.3. Справедливы следующие соотношения:

$$
\begin{aligned}
& \sqrt{24(1-x)} \leqslant \alpha(x) \leqslant \sqrt{\frac{24(1-x)}{x}}, \quad x \in(0,1], \\
& \sqrt{\frac{24(c-1)}{c}} \leqslant \beta(c) \leqslant \sqrt{24(c-1)}, \quad c \in[1,+\infty), \\
& 0 \leqslant B(c) \leqslant c^{2} \sqrt{\frac{c-1}{6}}, \quad c \in[1,+\infty), \\
& \frac{1}{2 \pi} \leqslant S(c)<\frac{(c+1)^{2}}{4 \pi}, \quad c \in[1,+\infty), \\
& S(c) \leqslant \frac{1}{2 \pi}+1.028 \frac{c^{2}}{\pi^{2}} \sqrt{\frac{8(c-1)}{3}}, \quad c \in\left[1,1+6 \cdot 10^{-5}\right] \text {, } \\
& S(c)>\frac{(c+1)^{2}}{4 \pi}\left(\frac{\pi /(c+1)}{\arcsin (\pi /(c+1))}\right)^{2}, \quad c \in[\pi-1,+\infty) \text {. }
\end{aligned}
$$


ДокАзАтельство. а) Из (2.7) и ряда Тейлора для $\sin z$ следует, что

$$
x=1-\frac{\alpha^{2}}{24} \cdot\left(1-\frac{\vartheta \alpha^{2}}{80}\right)
$$

где $\vartheta=\vartheta(\alpha) \in(0,1), \alpha \in[0,2 \pi]$. Положим

$$
\eta=\eta(\alpha):=\vartheta(\alpha) \frac{\alpha^{2}}{80}, \quad 0<\vartheta(\alpha)<1 .
$$

Очевидно, что $0<\eta<1 / 2, \alpha \in[0,2 \pi], \eta<1.25 \cdot 10^{-3}, \alpha \in[0, \pi / 10], \eta<$ $1.25 \cdot 10^{-6}, \alpha \in\left[0,10^{-2}\right]$. В частности, отсюда получаем, что

$$
\sqrt{24(1-x)} \leqslant \alpha(x) \leqslant \sqrt{48(1-x)}, \quad x \in[0,1] .
$$

Покажем, что при любом $x \in(0,1]$ верно неравенство $\alpha=\alpha(x) \leqslant \sqrt{24(1-x) / x}$. Сначала отметим его эквивалентность неравенствам $x \alpha^{2} \leqslant 24(1-x)$ и

$$
2 \sin \frac{\alpha}{2} \cdot \alpha^{2} \leqslant 24\left(\alpha-2 \sin \frac{\alpha}{2}\right), \quad \alpha \in[0,2 \pi)
$$

(см. (2.7)). Пусть $t=\alpha / 2$. Остается показать, что $g(t):=t^{2} \sin t \leqslant h(t):=$ $6(t-\sin t)$ при всех $t \in[0, \pi)$. Справедливость этого неравенства следует из соотношений

$$
\begin{gathered}
g(0)=h(0)=g^{\prime}(0)=h^{\prime}(0)=0, \\
g^{\prime \prime}(t)=\left(2-t^{2}\right) \sin t+4 t \cos t \leqslant 6 \sin t=h^{\prime \prime}(t), \quad x \in(0,1] .
\end{gathered}
$$

При $t \in[0, \pi / 2)$ последнее неравенство эквивалентно простому неравенству $4 t /\left(4+t^{2}\right) \leqslant \operatorname{tg} t$, при $t \in[\pi / 2, \pi)$ неравенство $g^{\prime \prime}(t) \leqslant h^{\prime \prime}(t)$ очевидно, так как $4 t \cos t \leqslant 0 \leqslant\left(t^{2}+4\right) \sin t$. Этим завершается доказательство второго из неравенств (2.13).

b) Эти неравенства следуют из неравенств (2.13) и соотношений (2.7).

c) При $c \in[1,+\infty)$ и $\beta=\beta(c) \in[0,2 \pi)$ справедливо неравенство $0 \leqslant \beta-$ $\sin \beta \leqslant \beta^{3} / 6$. Отсюда и из (2.14) получаем соотношения

$$
0 \leqslant B(c)=c^{2} \frac{\beta(c)-\sin \beta(c)}{2 \beta^{2}(c)} \leqslant c^{2} \frac{\beta(c)}{12} \leqslant c^{2} \sqrt{\frac{c-1}{6}} .
$$

d) При $c \in[1,+\infty)$ из $(2.15)$ и (2.11) получаем неравенства

$$
\frac{1}{2 \pi} \leqslant \frac{1}{2 \pi}+\frac{4 B(c)}{\pi^{2}}=S(c, \pi) \leqslant S(c) .
$$

Далее, так как в обозначениях леммы 2.2 при $l=1$ имеем $S(c, \alpha)=S_{1}^{*}+S_{2}^{*}$, то из неравенства (2.6) леммы 2.1 при любом $x \in[0,1]$ получаем неравенство $S(c, \alpha(x)) \leqslant(c x+1)^{2} /(4 \pi)$. Отсюда при $x \in[0,1)$ и $\alpha=\alpha(x) \in(0,2 \pi]$ получаем $S(c, \alpha)<(c+1)^{2} /(4 \pi)$. При $x=1$ сегмент $\operatorname{Seg}_{1}$ (см. обозначения леммы 2.2) вырождается в отрезок и потому $S_{1}^{*}=0$. Сегмент же $\operatorname{Seg}_{2}$ при $x=1$ имеет 
периметр $c+1$ и, следовательно, из изопериметрического неравенства получаем $S(c, \alpha(1))=S(c, 0)=S_{2}^{*}<(c+1)^{2} /(4 \pi)$. Поэтому

$$
S(c)=\max \{S(c, \alpha): \alpha \in[0,2 \pi]\}<\frac{(c+1)^{2}}{4 \pi}
$$

при любом $c \geqslant 1$.

е) Сначала оценим $S(c, \alpha)$ при $c \searrow 1$.

e1) Так как функции $(\alpha-\sin (\alpha)) /\left(2 \alpha^{2}\right)$ и $\sin (\alpha / 2) /(\alpha / 2)$ строго убывают на отрезке $[\pi, 2 \pi]$, то при $\alpha \in[\pi, 2 \pi]$ получаем неравенства

$$
\frac{\alpha-\sin (\alpha)}{2 \alpha^{2}} \leqslant \frac{1}{2 \pi}, \quad B(c)\left(\frac{\sin (\alpha / 2)}{\alpha / 2}\right)^{2} \leqslant \frac{4 B(c)}{\pi^{2}} .
$$

Отсюда при $\alpha \in[\pi, 2 \pi]$ имеем неравенство

$$
S(c, \alpha) \leqslant S(c, \pi)=\frac{1}{2 \pi}+\frac{4 B(c)}{\pi^{2}} .
$$

е2) При $\alpha \in[0,5 \pi / 6]$ функция $(\alpha-\sin (\alpha)) /\left(2 \alpha^{2}\right)$ возрастает. Следовательно, при таких $\alpha$ имеем

$$
\frac{\alpha-\sin (\alpha)}{2 \alpha^{2}} \leqslant \frac{5 \pi / 6-1 / 2}{50 \pi^{2} / 36}=\frac{6 / 5-36 /(50 \pi)}{2 \pi} \leqslant \frac{0.98}{2 \pi} .
$$

Кроме того,

$$
B(c)\left(\frac{\sin (\alpha / 2)}{\alpha / 2}\right)^{2} \leqslant B(c) .
$$

Таким образом, при условиях $\alpha \in[0,5 \pi / 6]$ и $B(c)\left(1-4 / \pi^{2}\right)<0.02 /(2 \pi)$ имеем также неравенство

$$
S(c, \alpha) \leqslant \frac{1}{2 \pi}+\frac{4 B(c)}{\pi^{2}}
$$

- такое же неравенство как и при $\alpha \in[\pi, 2 \pi]$. Указанное условие на $B(c)$ выполнено, например, при $B(c)<0.005$ (это легко проверяется простым подсчетом).

е3) Наконец, если $\alpha \in(5 \pi / 6, \pi)$, то $\alpha=\pi-\theta$, где $\theta=\theta(\alpha) \in(0, \pi / 6)$. Тогда

$$
\frac{1}{2 \pi}-\frac{\alpha-\sin (\alpha)}{2 \alpha^{2}}=\frac{(\pi-\theta)^{2}-\pi(\pi-\theta-\sin (\theta))}{2 \pi(\pi-\theta)^{2}}=\frac{\pi(\sin (\theta)-\theta)+\theta^{2}}{2 \pi(\pi-\theta)^{2}} .
$$

Так как $\sin (\theta)-\theta>-\theta^{3} / 6$ и $(\pi-\theta)^{2}<\pi^{2}$ при рассматриваемых $\theta$, то, используя оценку $\pi^{2}<10$, получаем неравенства

$$
\frac{1}{2 \pi}-\frac{\alpha-\sin (\alpha)}{2 \alpha^{2}}>\frac{-\pi \theta^{3} / 6+\theta^{2}}{2 \pi^{3}}>\frac{\theta^{2}\left(1-\pi^{2} / 36\right)}{2 \pi^{3}}>\frac{13 \theta^{2}}{36 \pi^{3}} .
$$

Отсюда следует, что

$$
\frac{\alpha-\sin (\alpha)}{2 \alpha^{2}}<\frac{1}{2 \pi}-\frac{13 \theta^{2}}{36 \pi^{3}} .
$$

Далее оценим сверху величину $B(c)(\sin (\alpha / 2) /(\alpha / 2))^{2}$ при $\alpha \in(5 \pi / 6, \pi)$. Отметим, что при всех $t \in(0, \pi / 6)$ верно неравенство

$$
f(t):=\cos (t)<h(t):=1-\frac{4 t^{2}}{\pi^{2}}=\left(1+\frac{2 t}{\pi}\right)\left(1-\frac{2 t}{\pi}\right)
$$


(это следует из того, что $f(0)=h(0)=1, f^{\prime}(0)=h^{\prime}(0)=0$ и $f^{\prime \prime}(t)=-\cos (t)<$ $-\sqrt{3} / 2<-8 / \pi^{2}=h^{\prime \prime}(t)$ при $\left.t \in(0, \pi / 6)\right)$. При $t=\theta / 2 \in(0, \pi / 12)$ отсюда получаем, что

$$
B(c)\left(\frac{\sin (\alpha / 2)}{\alpha / 2}\right)^{2}=4 B(c) \frac{\cos ^{2}(\theta / 2)}{(\pi-\theta)^{2}}=\frac{4 B(c)}{\pi^{2}} \frac{\cos ^{2}(\theta / 2)}{(1-\theta / \pi)^{2}}<4 B(c) \frac{(1+\theta / \pi)^{2}}{\pi^{2}} .
$$

Отсюда

$$
\begin{aligned}
& S(c, \alpha)= \frac{\alpha-\sin (\alpha)}{2 \alpha^{2}}+B(c)\left(\frac{\sin (\alpha / 2)}{\alpha / 2}\right)^{2}, \\
& S(c, \alpha)<\frac{1}{2 \pi}-\frac{13 \theta^{2}}{36 \pi^{3}}+\frac{4 B(c)(1+\theta / \pi)^{2}}{\pi^{2}}=\left(\frac{1}{2 \pi}+\frac{4}{\pi^{2}} B(c)\right) \\
& \quad+\frac{8}{\pi^{3}} B(c) \theta-\left(\frac{13}{36 \pi^{3}}-\frac{4}{\pi^{4}} B(c)\right) \theta^{2}=: F_{c}(\theta) .
\end{aligned}
$$

Если $B(c)<\pi / 144$, то квадратный трехчлен $F_{c}(\theta)$ имеет отрицательный старший коэффициент и достигает своего максимума, если

$$
\theta=\theta_{0}:=\frac{144 \pi B(c)}{13 \pi-144 B(c)}<12 B(c) .
$$

Отсюда получаем неравенства

$$
S(c, \alpha)<F_{c}(\theta) \leqslant F_{c}\left(\theta_{0}\right)<\frac{1}{2 \pi}+\frac{4 B(c)}{\pi^{2}}+\frac{8 B(c)}{\pi^{3}} \theta_{0}<\frac{1}{2 \pi}+\frac{4 B(c)}{\pi^{2}}+\frac{96 B^{2}(c)}{\pi^{3}}
$$

при $B(c)<0.005(0.005<\pi / 144)$.

Учитывая доказанные в пунктах е1)-е3) оценки для $S(c, \alpha)$, при $B(c)<0.005$ получаем двойное неравенство

$$
\frac{1}{2 \pi}+\frac{4}{\pi^{2}} B(c) \leqslant S(c) \leqslant \frac{1}{2 \pi}+\frac{4}{\pi^{2}} B(c)\left(1+\frac{24}{\pi} B(c)\right) .
$$

Отсюда и из неравенства $(2.15)$ при $1 \leqslant c \leqslant 1.00006$ получаем неравенства

$$
B(c) \leqslant c^{2} \sqrt{\frac{c-1}{6}}<0.0035<0.005, \quad S(c)<\frac{1}{2 \pi}+1.028 \frac{c^{2}}{\pi^{2}} \sqrt{\frac{8(c-1)}{3}} .
$$

f) Для доказательства (2.18) положим $\beta=\beta(c), \alpha_{0}=\alpha_{0}(c)=2 \pi-\beta$. Тогда $S(c) \geqslant S\left(c, \alpha_{0}\right)$. При $\alpha=\alpha_{0}$ область $G$ (см. лемму 2.2) представляет собой круг с периметром $c x_{0}+1, x_{0}=x\left(\alpha_{0}\right)$, и площадью $\left(c x_{0}+1\right)^{2} /(4 \pi)$. Следовательно,

$$
4 \pi \frac{S(c)}{(c+1)^{2}} \geqslant \frac{\left(c x_{0}+1\right)^{2}}{(c+1)^{2}} .
$$

Оценим снизу величину $c x_{0}+1$. Так как $\beta=2 \pi-\alpha_{0}, x_{0}=2 \sin \left(\alpha_{0} / 2\right) / \alpha_{0}$ и $c=\beta /(2 \sin (\beta / 2))$, то

$$
c x_{0}+1=2 c \frac{\sin \left(\alpha_{0} / 2\right)}{\alpha_{0}}+1=2 c \frac{\sin (\beta / 2)}{\alpha_{0}}+1=\frac{\beta}{\alpha_{0}}+1=\frac{2 \pi}{\alpha_{0}} .
$$


Остается оценить сверху величину $\alpha_{0}$. Очевидно,

$$
c=\frac{\beta}{2 \sin (\beta / 2)}=\frac{2 \pi-\alpha_{0}}{2 \sin \left(\alpha_{0} / 2\right)} .
$$

Отсюда

$$
2 c \sin \frac{\alpha_{0}}{2}=2 \pi-\alpha_{0} \leqslant 2 \pi-2 \sin \frac{\alpha_{0}}{2}, \quad(c+1) \sin \frac{\alpha_{0}}{2} \leqslant \pi
$$

и, следовательно, $\alpha_{0} \leqslant 2 \arcsin (\pi /(c+1))$ при всех $c>\pi-1$. Поэтому

$$
4 \pi \frac{S(c)}{(c+1)^{2}} \geqslant \frac{\left(c x_{0}+1\right)^{2}}{(c+1)^{2}}=\left(\frac{2 \pi}{\alpha_{0}(c+1)}\right)^{2} \geqslant\left(\frac{\pi /(c+1)}{\arcsin (\pi /(c+1))}\right)^{2} .
$$

Этим заканчивается доказательство леммы 2.3.

Подведем итоги сделанного в леммах 2.2 и 2.3 .

Сначала с использованием принципа Дидоны были получены аналитические выражения для величин $S(c, \alpha), \alpha \in[0,2 \pi]$, и $\widetilde{S}(c, x), x \in[0,1]$, выражающих общий максимум площадей $\operatorname{mes}_{2}(G)$ всех ограниченных областей $G$ со спрямляемыми жордановыми границами, удовлетворяющими условиям леммы 2.2 при каком-либо фиксированном наборе параметров $l=|L|>0,|j|=|b-a|=x l$, $x \in[0,1]$, и $c \geqslant 1(x$ и $\alpha=\alpha(x)$ связаны первым уравнением из $(2.7) ; \widetilde{S}(c, x)=$ $S(c, \alpha(x))$, см. (2.9), (2.10)). Затем в лемме 2.3 аналитическими методами были получены асимптотические неравенства для функций $\alpha=\alpha(x)$ при $x \searrow 0$ и $x \nearrow 1$, а также для $\beta(c)=\alpha(1 / c), B(c)$ и $S(c)$ (см. (2.8) и (2.11)) при $c \searrow 1$ и c $\nearrow+\infty$.

Лемма 2.4. Пусть граница $\partial G$ ограниченной области $G$ является замкнутой жордановой спрямляемой кривой, состоящей из дуг $L$ и $\Sigma$ с общими кониами $a, b \in \mathbb{C}, j:=[a, b]$ - общая хорда этих дуг (допускается случай $a=b$, когда $\Sigma$ и ј вырождаются в точку $a=b)$, причем

$$
|L|=: l>0, \quad|j|=x l, \quad 0 \leqslant x:=\frac{|j|}{l} \leqslant 1, \quad|\Sigma| \leqslant c|j|
$$

при некотором $c=$ const $\geqslant 1$. Тогда (см. (2.9)-(2.11); числа $\alpha \in[0,2 \pi] u$ $x \in[0,1]$ связаны первым уравнением из $(2.7))$ :

$$
\begin{gathered}
\operatorname{mes}_{2}(G) \leqslant S(c, \alpha) l^{2}=\widetilde{S}(c, x) l^{2} \leqslant S(c) l^{2} \leqslant \bar{S}(c) l^{2}=\frac{(c+1)^{2}}{8 \pi} \bar{s}(c) l^{2}, \\
\bar{s}(c):=1+\frac{\sqrt{1-c^{-8 / 3}}}{1+(1.19-0.67 / c) c^{-8 / 3}}, \quad c \in[1,25], \quad \bar{s}(c):=2, \quad c>25 .
\end{gathered}
$$

Относительная погрешность $((\bar{S}(c)-S(c)) / S(c)) \cdot 100 \%$ от замены в (2.19) функции $S(c)$ функцией $\bar{S}(c)$ строго менвще $0.65 \%$ при всех $c \geqslant 1$, а при с \1 и при с $\nearrow+\infty$ она стремится к нулю.

ЗАмечАниЕ 2.2. О точности первых двух неравенств в (2.19) см. замечание 2.1. О степени точности последнего из неравенств (2.19) сказано в конце формулировки леммы 2.4 . 
ЗАмЕчАнИЕ 2.3. Выражение функции $S(c)$ (см. (2.11)) в виде элементарной функции от $B(c)$ (см. (2.8) и (2.7)) получила обычным способом (путем решения уравнения $\left.S_{\alpha}^{\prime}(c, \alpha)=0\right)$ моя ученица - студентка Е.В. Абрамова. Вот это простое равенство:

$$
S(c) \equiv \frac{1}{4 \operatorname{arcctg}(4 B(c))} .
$$

ДокАЗАТЕЛЬСтво. Приняв во внимание лемму 2.2, будем доказывать лишь неравенство $S(c) \leqslant \bar{S}(c)$ и указанную оценку для относительного уклонения $\bar{S}(c)$ от $S(c), c \geqslant 1$. Как и при доказательстве леммы 2.2 , будем пользоваться обозначениями начала п. 2.6, а также леммы 2.1 и первой части ее доказательства.

Начнем с описания того, как была подобрана неубывающая функция $\bar{s}(c)$, $c \geqslant 1$, и соответствующая строго возрастающая функция $\bar{S}(c)$ (см. (2.19) и (2.20)), обладающие следующими свойствами:

I) функция $\bar{s}(c)$ на $[1,25]$ непрерывно строго возрастает от $\bar{s}(1)=1$ до $\bar{s}(25)<2, \bar{s}(c) \equiv 2$ при $c>25$; соответственно, при $c \geqslant 1$ функция $\bar{S}(c)$ строго возрастает от $\bar{S}(1)=S(1)=1 /(2 \pi)$ до $+\infty$, причем на $[1,25]$ она непрерывна, $\bar{S}(c) \equiv 2(c+1)^{2} /(8 \pi)$ при $c>25$, а в точке $c=25$ она имеет разрыв справа: при переходе $c$ через эту точку $\bar{S}(c)$ "подпрыгивает" от значения $\bar{S}(25)<2(25+1)^{2} /(8 \pi)$ до значения $\bar{S}(25+)=2(25+1)^{2} /(8 \pi)$;

II) при всех $c \in(1,+\infty)$ выполнено неравенство $\bar{S}(c)>S(c)$, кроме того, $\bar{S}(c) / S(c) \rightarrow 1$ при $c \searrow 1$ и при $c \nearrow+\infty$ (так что $\bar{S}(1)=S(1)=1 /(2 \pi)$ и $\bar{S}(+\infty)=S(+\infty)=+\infty)$;

III) функция $\bar{S}(c)$ достаточно хорошо приближает (сверху) функцию $S(c)$, а именно, равномерное относительное уклонение ее от $S(c)$ при всех $c \geqslant 1$ строго меньше $0.65 \%$ :

$$
\rho(S, \bar{S}):=\sup \left\{\frac{\bar{S}(c)-S(c)}{S(c)}=\frac{\bar{S}(c)}{S(c)}-1: c \geqslant 1\right\}<6.5 \cdot 10^{-3} .
$$

При этом $\bar{S}(c)$ конструировалась из элементарных функций и подбиралась так, чтобы в разумной мере удовлетворялись следующие два требования, в некотором смысле противоречащие друг другу: во-первых, чтобы величина $\rho(S, \bar{S})$ была поменьше, и, во-вторых, чтобы функция $\bar{S}(c)$ была попроще. Явное выражение для $\bar{S}(c)$ указано в формулировке леммы 2.4. Для нас здесь не важно, каким образом оно было найдено, а важно доказать, что $\bar{S}(c)$ обладает всеми свойствами, указанными в заключении леммы. Далее об этом более подробно.

Из (2.16)-(2.18) леммы 2.3 и из (2.22) следует, что для функций

$$
s(c):=8 \pi(c+1)^{-2} S(c), \quad \bar{s}(c)=8 \pi(c+1)^{-2} \bar{S}(c)
$$

при $c \geqslant 1$ верны следующие соотношения:

$$
s(1)=\lim _{c^{\prime} \searrow 1} s\left(c^{\prime}\right)=1, \quad s(c)<\lim _{c^{\prime} \nearrow+\infty} s\left(c^{\prime}\right)=2 ; \quad \rho(s, \bar{s})=\rho(S, \bar{S}) .
$$

Требование $\bar{s}(c) \equiv 2$ для всех $c>25$ объясняется следующими соображениями.

Именно, делается это для простоты и надежности вычисления $\bar{s}(c)$ (и тем самым вычислений величины $s(c)$ с указанным в лемме 2.4 ограничением $0.65 \%$ 
для допустимой погрешности) при больших $c$. Этим приемом был также учтен и тот факт, что при больших $c$ величина $\bar{s}(c)-s(c)=2-s(c)$ уже мала, в то время как ошибки округления в численно найденных значениях $s(c)$ велики.

Для подбора подходящих функций $\bar{s}(c)$ и $\bar{S}(c)$ использовался компьютер. Именно, на некоторой сетке $1=c_{0}<1+3 \cdot 10^{-5}=c_{1}<c_{2}<\cdots<c_{n}=25$ (неравномерной, в дальнейшем подбираемой) с помощью программ Maple и Mathematica были последовательно посчитаны значения функций $\beta\left(c_{k}\right), B\left(c_{k}\right)$ и, наконец, $S\left(c_{k}\right)$ (см. $(2.7),(2.8),(2.10)$ и $\left.(2.11)\right)$. После этого по упомянутой таблице значений строго возрастающей функции $S(c)$, учитывая ее асимптотики при $c \searrow 1$ и $c \nearrow+\infty$ (лемма 2.3), подбирались функции $\bar{s}(c)$ и $\bar{S}(c)$ со свойствами, перечисленными в пп. I)-III). Для этого функции $\bar{s}(c)$ и $\bar{S}(c)$ (и сетки $\left.\left\{c_{k}\right\}\right)$ строились при постоянном контроле соблюдения двух серий строгих неравенств при $k=2,3, \ldots, n$ :

$$
\text { E) } \left.\bar{S}\left(c_{k-1}\right)>S\left(c_{k}\right)+10^{-5}, \quad F\right) \quad \frac{\bar{S}\left(c_{k}\right)}{S\left(c_{k-1}\right)}-1<6.4 \cdot 10^{-3} .
$$

Для функции $\bar{S}(c) \equiv(c+1)^{2} \bar{s}(c) /(8 \pi)$, где $\bar{s}(c)$ определяется равенствами $(2.20)$, удалось так подобрать $n \approx 1000$ различных узлов сетки $\left\{c_{k}\right\}$, чтобы оба неравенства из (2.23) были выполнены при всех $k=2,3, \ldots, n$.

Отметим, что для дальнейшего вместо неравенств (2.23) нам достаточно иметь более слабые неравенства $\bar{S}\left(c_{k-1}\right)>S\left(c_{k}\right)$ и $\bar{S}\left(c_{k}\right) / S\left(c_{k-1}\right)-1<6.5 \cdot 10^{-3}$, $k=2,3, \ldots, n$. Следование же усиленным неравенствам (2.23) при подборе функции $\bar{S}(c)$ нужно для уверенности в том, что ошибки округления при компьютерных вычислениях входящих в них величин не повлияют на справедливость этих слабых неравенств.

Докажем, что такие функции $\bar{s}(c)$ и $\bar{S}(c)$ из (2.20) и (2.19) обладают всеми свойствами, перечисленными в пп. I)-III), откуда будут следовать оставшиеся пока еще не доказанными утверждения леммы 2.4.

Во-первых, утверждения п. I) очевидны. Дальнейшее доказательство разобьем на три части, в зависимости от величины $c$ :

1) $c \in\left[c_{1}=1+3 \cdot 10^{-5}, c_{n}=25\right]$;

2) $1 \leqslant c \leqslant c_{1}=1+3 \cdot 10^{-5}$;

3) $c>c_{n}=25$.

1) Пусть $c \in\left[c_{1}, c_{n}\right]$. Возьмем такое натуральное $k$, что $c \in\left[c_{k-1}, c_{k}\right]$. Тогда из неравенств $(2.23), \mathrm{E})$ и строгого возрастания функций $\bar{S}(c)$ и $S(c)$ при $c \geqslant 1$ вытекают неравенства $\bar{S}(c) \geqslant \bar{S}\left(c_{k-1}\right)>S\left(c_{k}\right)+10^{-5} \geqslant S(c)$. Кроме того, при этом же $c$ из $(2.23), \mathrm{F})$ получаем неравенства

$$
\frac{\bar{S}(c)}{S(c)}-1<\frac{\bar{S}\left(c_{k}\right)}{S\left(c_{k-1}\right)}-1<6.5 \cdot 10^{-3} .
$$

Итак, справедливы неравенства

$$
\bar{S}(c)>S(c), \quad \frac{\bar{S}(c)-S(c)}{S(c)}<6.5 \cdot 10^{-3}, \quad c \in\left[c_{1}, c_{n}\right] .
$$

2) Пусть $1=c_{0} \leqslant c \leqslant c_{1}=1+3 \cdot 10^{-5}$. Оценим снизу $\bar{s}(c)$ при этих $c$. Так как при таких $c$ имеем $c^{8 / 3} \geqslant 1+(8 / 3)(c-1)$, то

$$
\sqrt{1-c^{-8 / 3}}=c^{-4 / 3} \sqrt{c^{8 / 3}-1} \geqslant c^{-4 / 3} \sqrt{\frac{8}{3}(c-1)} .
$$


Покажем, что

$$
G(c):=1.19 c<0.67+0.52 c^{11 / 3}=: H(c) .
$$

Имеем $G(1)=H(1)$ и $G^{\prime}(c)=1.19<11 \cdot 0.52 / 3 \leqslant 11 \cdot 0.52 c^{8 / 3} / 3=H^{\prime}(c)$. Отсюда получаем

$$
\begin{gathered}
c^{11 / 3}+1.19 c-0.67 \leqslant 1.52 c^{11 / 3} \\
1+\left(1.19-\frac{0.67}{c}\right) c^{-8 / 3}=\left(c^{11 / 3}+1.19 c-0.67\right) c^{-11 / 3} \leqslant 1.52 .
\end{gathered}
$$

Поэтому

$$
\begin{gathered}
\bar{s}(c) \geqslant 1+\frac{c^{-4 / 3} \sqrt{8(c-1) / 3}}{1.52} \\
\frac{(c+1)^{2} \bar{s}(c)}{8 \pi} \geqslant \frac{c \bar{s}(c)}{2 \pi} \geqslant \frac{c}{2 \pi}+\frac{c^{-1 / 3} \sqrt{8(c-1) / 3}}{3.04 \pi} .
\end{gathered}
$$

При $1 \leqslant c \leqslant c_{1}=1.00003$ получаем $c^{7 / 3} \leqslant c^{3} \leqslant 1.0002<\pi /(1.028 \cdot 3.04)$. Отсюда и из $(2.17)$ при $c \in\left(1, c_{1}\right]$ получаем неравенства

$$
\frac{(c+1)^{2}}{8 \pi} \bar{s}(c)-S(c) \geqslant \frac{c-1}{2 \pi}+\frac{c^{2}}{\pi^{2}} \sqrt{\frac{8(c-1)}{3}}\left(\frac{\pi}{3.04} c^{-7 / 3}-1.028\right)>0,
$$

обращающиеся в равенства при $c=1$.

Этим неравенство (2.19) доказано при $c \in\left[c_{0}, c_{1}\right]$. Покажем теперь, что равномерное относительное уклонение функции $\bar{S}(c)$ от $S(c)$ при этих $c$ строго меньше $6.5 \cdot 10^{-3}$. Действительно, из неравенства $(2.16)$ и монотонного возрастания функции $\bar{S}(c) \geqslant S(c)$ на отрезке $\left[1,1+3 \cdot 10^{-5}\right]$ получаем следующие неравенства: $1 \leqslant \bar{S}(c) / S(c) \leqslant 2 \pi \bar{S}(c) \leqslant 2 \pi \bar{S}\left(1+3 \cdot 10^{-5}\right)<1.006$. Отсюда вытекает требуемое.

3) Пусть $c>c_{n}=25$. Напомним, что при таких $c$ имеем тождества $\bar{s}(c) \equiv 2$ и $\bar{S}(c) \equiv(c+1)^{2} /(4 \pi)$.

Неравенство $\bar{S}(c)>S(c)$ при $c>25$ доказано в лемме 2.3 (см. (2.16)). Покажем, что если $c>c_{n}$, то равномерное относительное уклонение функции $\bar{S}(c)$ от $S(c)$ не превосходит $5 \cdot 10^{-3}$. Действительно, из $(2.17)$ при $t=\arcsin (\pi /(c+1))$ следует неравенство $(c+1)^{2} /(4 \pi S(c)) \leqslant(t / \sin t)^{2}$. Так как функция $t / \sin t$ возрастает при $t \in[0,1]$, а функция $t=\arcsin (\pi /(c+1))$ убывает при $c>25$, то при этих $c$ имеем следующие соотношения:

$$
1<\frac{\bar{S}(c)}{S(c)}=\frac{(c+1)^{2}}{4 \pi S(c)}<\left(\frac{\arcsin (\pi / 26)}{\pi / 26}\right)^{2}<1.005
$$

что и требовалось. Лемма 2.4 доказана.

2.7. Оценки интеграла $F(r)$. Следующее утверждение, интересное и само по себе, понадобится при доказательстве леммы 2.7 .

Лемма 2.5. Пусть $G$ - односвязная область плоскости $\mathbb{C}, \tau-$ достижимая точка ее границы $\partial G, w=\psi(z)$ - однолистное конформное отображение единичного круга $D$ на $G, t=\psi^{-1}(\tau)$ - прообраз достижимой точки $\tau$ 
при этом отображении $(t \in C=\partial D)$; положительное число $r_{0}$ таково, что $\psi\left(\Delta_{t}\left(r_{0}\right)\right)$ - ограниченная подобласть области $G$ (см. n. 1.4). Тогда на любом замкнутом множестве $E \subset\left(0, r_{0}\right)$ найдется число $\widehat{r}=r(\psi, t, E) \in E$, для которого $l_{t}(\widehat{r})=m(E):=\inf \left\{l_{t}(r): r \in E\right\}$. В частности, функиия $l_{t}(r)$ переменного $r \in\left(0, r_{0}\right)$ полунепрерьвна снизу в каждой точке интервале $\left(0, r_{0}\right)$.

ДокАЗАтЕЛьство. Пусть $v_{n} \in E, n=1,2, \ldots$, таковы, что $l_{t}\left(v_{n}\right)<m(E)+$ $1 / n$ для всех $n$. Можно считать, что $v_{n} \rightarrow \widehat{r}$ при некотором $\widehat{r} \in E$ и $n \rightarrow \infty$. Покажем, что $\widehat{r}-$ нужное число.

Отображение $\zeta=L(z):=1-z / t$ поворачивает плоскость $\mathbb{C}$ вокруг точки нуль, а затем параллельно сдвигает плоскость так, что $L(t)=0, L(D=$ $D(0,1))=D(1,1)$, а дуги $\lambda_{t}(v), 0<v<2$, переходят в круговые дуги $L\left(\lambda_{t}(v)\right)$ (с центром в нуле, радиусами $v$ и длинами $l_{t}(v)$ соответственно), симметричные относительно действительной оси. Положим

$$
\begin{gathered}
\lambda:=L\left(\lambda_{t}(\widehat{r})\right), \quad l:=\left|\psi\left(L^{-1}(\lambda)\right)\right|=l_{t}(\widehat{r}), \quad \lambda_{n}:=L\left(\lambda_{t}\left(v_{n}\right)\right), \\
l_{n}:=\left|\psi\left(L^{-1}\left(\lambda_{n}\right)\right)\right|=l_{t}\left(v_{n}\right), \quad k_{n}:=\frac{\left|\lambda_{n}\right|}{|\lambda|} .
\end{gathered}
$$

Тогда

$$
\begin{gathered}
l=\int_{\lambda}\left|t \psi^{\prime}\left(L^{-1}(\zeta)\right)\right||d \zeta|=\int_{\lambda}\left|t \psi^{\prime}\left(L^{-1}(\zeta)\right)\right| \widehat{r} d \arg \zeta \\
l_{n}=\int_{\lambda_{n}}\left|t \psi^{\prime}\left(L^{-1}(\zeta)\right)\right| v_{n} d \arg \zeta=\int_{\lambda} \frac{v_{n}}{\widehat{r}}\left|t \psi^{\prime}\left(L^{-1}\left(v_{n} e^{i k_{n} \arg \zeta}\right)\right)\right| \widehat{r} d \arg \zeta .
\end{gathered}
$$

Так как $v_{n} \rightarrow \widehat{r}$ и $k_{n} \rightarrow 1$ при $n \rightarrow \infty$, то в каждой неконцевой точке $\zeta$ дуги $\lambda$ выражение под знаком последнего интеграла в последней формульной строке положительно и при $n \rightarrow \infty$ стремится к положительному выражению под знаком второго интеграла в предпоследней формульной строке. Из этого и стремления $l_{n} \rightarrow m(E)$ в силу леммы Фату (см., например, [15; гл. $\left.1, \S 12\right]$ ) вытекает неравенство $m(E) \geqslant l$. А так как $m(E) \leqslant l$ (по определению $m(E))$, то $m(E)=l$, что и требовалось.

Лемма 2.6. При любых фиксированных значениях $X>0 u \lambda \in(-\infty,+\infty)$ величина $U^{-1}(a ; U(a ; X)+\lambda)$ не зависит от числа а или, что то же самое,

$$
U^{-1}(a ; U(a ; X)+\lambda) \equiv U^{-1}(1 ; U(1 ; X)+\lambda), \quad X>0, \quad \lambda \in(-\infty,+\infty) .
$$

ДокАЗАТЕЛЬСтво. Пусть

$$
b:=\int_{a}^{1} \frac{d y}{u^{-1}(y)} .
$$

Если $Y:=U(a ; X)$, то $U(1 ; X)=Y-b$. Из этих двух равенств вытекают равенства $X=U^{-1}(a ; Y)$ и $X=U^{-1}(1 ; Y-b)$, откуда имеем тождество $U^{-1}(a ; Y) \equiv U^{-1}(1 ; Y-b)$, верное при всех $Y \in(-\infty,+\infty)$. При $Y=U(a ; X)+\lambda$ отсюда получаем, что

$$
U^{-1}(a ; U(a ; X)+\lambda) \equiv U^{-1}(1 ; U(a ; X)+\lambda-b) \equiv U^{-1}(1 ; U(1 ; X)+b+\lambda-b) .
$$

Лемма 2.6 доказана. 
Существенную роль в дальнейшем будет играть интеграл (1.5). Оценим его сверху и снизу. В обозначениях пп. 1.4, 1.5 справедлива следующая лемма.

Лемма 2.7. Пусть $G$ - односвязная область плоскости $\mathbb{C}, \Gamma$ - ограниченная открытая достижимая жорданова дуга ее границы $\partial G$ (в частности, может быть $\Gamma=\partial G), w=\psi(z)$ - однолистное конформное отображение единичного круга $D$ на $G, \underline{\gamma:=} \psi^{-1}(\Gamma), \gamma \subset C=\partial D, t \in \gamma, u$ при некотором числе $\rho \in(0,2)$ замыкание $\overline{\sigma_{t}(\rho)}$ дуги $\sigma_{t}(\rho):=\{z:|z|=1,|z-t|<\rho\}$ включается в $\gamma$. Если при этом функиия $y=u(x)$ определена и строго возрастает при $x \geqslant 0$ (она может быть разрывной), $u(0)=u(0+)=0, u(x) \geqslant x, x \geqslant 0, u$

$$
\pi S_{t}(r) \leqslant u\left(l_{t}^{2}(r)\right), \quad r \in[0, \rho],
$$

то для любого $r \in[0, \rho]$ имеем неравенство (см. (1.5), (1.6))

$$
\begin{gathered}
F(r) \leqslant U^{-1}\left(a ; U(a ; F(\rho))+\log \left(\frac{r}{\rho}\right)\right)=U^{-1}\left(a ; \log \left(E_{t}(\rho) r\right)\right), \\
E_{t}(\rho):=\rho^{-1} \exp \{U(a ; F(\rho))\} .
\end{gathered}
$$

При этом для любого $r \in(0, \rho]$ найдется такое число $\widehat{r}=\widehat{r}(\psi, t ; r) \in[r / e, r]$, что

$$
l_{t}(\widehat{r})<\sqrt{F(r)} \leqslant \sqrt{U^{-1}\left(a ; \log \left(E_{t}(\rho) r\right)\right)} .
$$

Выражение $U^{-1}(a ; U(a ; F(\rho))+\log (r / \rho))=U^{-1}\left(a ; \log \left(E_{t}(\rho) r\right)\right)$ в правых частях неравенств (2.25) и (2.27) на самом деле не зависит от величины $а>0$.

ЗАМЕЧАНИЕ 2.4. Видно, что

$$
E_{t}(\rho) \leqslant \rho^{-1} \exp \left\{U\left(a ; \pi S_{t}(\rho)\right)\right\}<\rho^{-1} \exp \left\{U\left(a ; \pi \operatorname{mes}_{2}(G)\right)\right\}, \quad a>0
$$

(при этом, возможно, $\left.\operatorname{mes}_{2} G=+\infty\right), E_{t}(\rho)=1 / \rho$ при $a=F(\rho)$ (см. (1.6)).

ДокАзАтЕльство. При $v \in[0,2]$, используя неравенство Коши-Буняковского, получаем

$$
l_{t}^{2}(v)=\left(\int_{\lambda_{t}(v)}\left|\psi^{\prime}(z)\right||d z|\right)^{2} \leqslant\left(\int_{\lambda_{t}(v)}\left|\psi^{\prime}(z)\right|^{2}|d z|\right) \cdot \operatorname{mes}_{1}\left(\lambda_{t}(v)\right) .
$$

Так как $\operatorname{mes}_{1}\left(\lambda_{t}(v)\right)<\pi v$ при $0<v<2$, то отсюда и из (1.5) следует простая оценка для $F(r)$ :

$$
\begin{gathered}
F(r)<\pi \int_{0}^{r} d v \cdot \int_{\lambda_{t}(v)}\left|\psi^{\prime}(z)\right|^{2}|d z|, \\
F(r)<\pi S_{t}(r), \quad r \in(0, \rho] .
\end{gathered}
$$

Функция $F(r)=F(\psi, t ; r)$ абсолютно непрерывна, поэтому из (1.5) для почти всех $r>0$ имеем равенства $F^{\prime}(r)=l_{t}^{2}(r) / r, l_{t}^{2}(r)=r F^{\prime}(r)$. Так как функции $u(r), u^{-1}(r)$ и $F(r)$ при $r>0$ положительны и не убывают, то из $(2.28)$ и условия (2.24) леммы получаем для почти всех $r \in(0, \rho]$ неравенства $F(r)<$ $\pi S_{t}(r) \leqslant u\left(l_{t}^{2}(r)\right)=u\left(r F^{\prime}(r)\right), u^{-1}(F(r))<r F^{\prime}(r)$ и, наконец,

$$
\frac{F^{\prime}(r)}{u^{-1}(F(r))}-\frac{1}{r}>0 \text {. }
$$


Следующая функция

$$
H(r):=-\int_{F(r)}^{F(\rho)} \frac{d x}{u^{-1}(x)}+\log \frac{\rho}{r}=-U(a ; F(\rho))+U(a ; F(r))+\log \frac{\rho}{r}
$$

(см. (1.6)) абсолютно непрерывна на каждом отрезке $[\eta, \rho]$ при $0<\eta<\rho$. Действительно, слагаемое $U(a ; F(r))$ на каждом таком отрезке представляет собой суперпозицию двух абсолютно непрерывных строго монотонных функций и поэтому само является абсолютно непрерывной функцией, а остальные слагаемые непрерывно дифференцируемы на таком отрезке. Так как $H^{\prime}(r)=$ $F^{\prime}(r) / u^{-1}(F(r))-1 / r>0$ почти для всех $r \in(0, \rho]$, то $H(r)$ является первообразной для функции $F^{\prime}(r) / u^{-1}(F(r))-1 / r$ и строго возрастает на $(0, \rho]$. Так как при этом $H(\rho)=0$, то $H(r)<0$ при $r \in(0, \rho)$. Отсюда следует неравенство $U(a ; F(r)) \leqslant U(a ; F(\rho))+\log (r / \rho)$ для всех $r \in[0, \rho]$, эквивалентное неравенству (2.25).

Докажем вторую часть леммы. В силу леммы 2.5 для любого $r \in(0, \rho]$ существует такое число $\widehat{r}=\widehat{r}(\psi, t ; r) \in[r / e, r]$, что $l_{t}(\widehat{r}) \leqslant l_{t}(v)$ для любого $v \in[r / e, r]$. Отсюда следует, что

$$
l_{t}^{2}(\widehat{r})=\int_{r / e}^{r} l_{t}^{2}(\widehat{r}) \frac{d v}{v} \leqslant \int_{r / e}^{r} l_{t}^{2}(v) \frac{d v}{v}<F(r) .
$$

Вместе с (2.25) это дает неравенство (2.27).

Наконец, независимость выражения $\sqrt{U^{-1}\left(a ; \log \left(E_{t}(\rho) r\right)\right)}$ от $а$ в неравенствах (2.25) и (2.27) вытекает из предыдущей леммы 2.6.

\section{8. Лемма о поведении конформных отображений круга на его радиусах.}

Лемма 2.8. Пусть $G$ - односвязная область плоскости $\mathbb{C}, \Gamma$ - ограниченная открытая достижимая жорданова дуга ее гранищы $\partial G, w=\psi(z)$ - однолистное конформное отображение единичного круга $D$ на $G, \gamma=\psi^{-1}(\Gamma) \subset$ $C=\partial D$. Тогда найдется такая точка $t=e^{i \tau} \in \gamma$, что функция

$$
\Psi_{t}(r):=\Psi(z=r t):=\int_{0}^{r}\left|\psi^{\prime}(s t)\right|^{2} s d s, \quad 0 \leqslant r \leqslant 1,
$$

возрастающая и абсолютно непрерывная на отрезке $[0,1]$, следующим образом оценивает сверху модуль непрерывности функиии $\psi(z)$ на отрезке $[t / 2, t]$ радиуса $R(t):=[0, t]$ круга $D$ и на самом этом радиусе при $\delta \in[0,1 / 2]$ :

$$
\begin{gathered}
\omega\left(\psi,\left[\frac{t}{2}, t\right], \delta\right) \leqslant \sqrt{2 \omega\left(\Psi,\left[\frac{1}{2}, 1\right], \delta\right)} \cdot \sqrt{\delta} \quad(=o(\sqrt{\delta}) n p u \delta \rightarrow 0), \\
\omega(\psi, R(t), \delta) \leqslant \Pi \sqrt{\delta} .
\end{gathered}
$$

Если при этом дуга Г спрямляема, то точку $t$ можно выбрать так, чтобъ для нее выполнялись также и следующие условия:
a) $\exists \lim _{R(t) \ni z \rightarrow t} \psi^{\prime}(z) \neq 0, \infty$;
b) $\omega(\psi, R(t), \delta) \leqslant \Pi_{1} \delta, \quad \delta \geqslant 0$.

Здесь конечные положительные величины $\Pi$ $и \Pi_{1}$ зависят от $\psi, \Gamma u t$, но не or $\delta$. 
ДоказАТЕЛЬСтво. Мы можем считать, что $\psi^{\prime}(z) \not \equiv$ const.

Пусть $\sigma:=\left\{e^{i \theta}: \tau^{\prime}<\theta<\tau^{\prime \prime}\right\}$ - какая-либо открытая дуга, содержащаяся в $\gamma$ вместе со своим замыканием $\bar{\sigma}$, а $Q:=\left\{z=r e^{i \theta}: \tau^{\prime}<\theta<\tau^{\prime \prime}, 1 / 2<r<1\right\}$. Тогда

$$
S:=\operatorname{mes}_{2}(\psi(Q))=\int_{\tau^{\prime}}^{\tau^{\prime \prime}} d \theta \int_{1 / 2}^{1}\left|\psi^{\prime}\left(r e^{i \theta}\right)\right|^{2} r d r<\infty
$$

При $t=e^{i \tau} \in \sigma$ и $1 / 2 \leqslant r^{\prime}<r^{\prime \prime} \leqslant 1$ положим

$$
\Delta_{r^{\prime}, r^{\prime \prime}} \Psi_{t}:=\Psi\left(r^{\prime \prime} t\right)-\Psi\left(r^{\prime} t\right)=\int_{r^{\prime}}^{r^{\prime \prime}}\left|\psi^{\prime}(s t)\right|^{2} s d s
$$

$M=$ const $>0, E(M)-$ множество (оно может оказаться пустым) всех точек $t \in \sigma$, для которых $\Delta_{1 / 2,1} \Psi_{t}>M$. Положим также $C E(M):=\sigma \backslash E(M)$, $|E(M)|:=\operatorname{mes}_{1}(E(M))$. Тогда $M|E(M)|<S$, откуда $|E(M)|<S / M$. Так как величина $S / M$ с ростом $M$ от нуля до $+\infty$ непрерывно убывает от $+\infty$ до нуля, то имеется ровно одно такое число $M>0$, что $S / M=|\sigma|$. Тогда $|E(M)|<|\sigma|,|C E(M)|>0$, и для любой точки $t=e^{i \tau} \in C E(M)$ верно неравенство $\Delta_{1 / 2,1} \Psi_{t}<M=S /|\sigma|$. Так как

$$
\left|\psi\left(r^{\prime \prime} t\right)-\psi\left(r^{\prime} t\right)\right|=\left|\int_{r^{\prime}}^{r^{\prime \prime}} \sqrt{s} \psi^{\prime}(s t) s^{-1 / 2} d s\right|
$$

то отсюда и из неравенства Коши-Буняковского получаем при $1 / 2 \leqslant r^{\prime}<$ $r^{\prime \prime} \leqslant 1$ неравенство

$$
\left|\psi\left(r^{\prime \prime} t\right)-\psi\left(r^{\prime} t\right)\right| \leqslant\left(\int_{r^{\prime}}^{r^{\prime \prime}} s\left|\psi^{\prime}(s t)\right|^{2} d s\right)^{1 / 2} \cdot\left(2\left|r^{\prime \prime}-r^{\prime}\right|\right)^{1 / 2},
$$

так как $1 / s \leqslant 2$. А это эквивалентно неравенству $(2.29)$. Неравенство же $(2.30)$ следует из $(2.29)$ и того, что при $\delta \leqslant 1 / 2$ имеем неравенства $\omega(\psi, R(t), \delta) \leqslant$ $\omega(\psi,[0, t / 2], \delta)+\omega(\psi,[t / 2, t], \delta)$, где, очевидно, $\omega(\psi,[0, t / 2], \delta) \leqslant M^{\prime} \delta \leqslant M^{\prime} \sqrt{\delta}$ при $M^{\prime}:=\max \left\{\left|\psi^{\prime}(z)\right|:|z| \leqslant 1 / 2\right\}$, а $\omega(\psi,[t / 2, t], \delta) \leqslant K \sqrt{\delta}$ (K не зависит от $\delta$; см. $(2.29))$. Таким образом, при $0 \leqslant \delta \leqslant 1$ имеем неравенство

$$
\omega(\psi, R(t), \delta) \leqslant 2 \omega\left(\psi, R(t), \frac{\delta}{2}\right) \leqslant 2 M^{\prime} \sqrt{\frac{\delta}{2}}+2 K \sqrt{\frac{\delta}{2}} \leqslant 2^{1 / 2}\left(M^{\prime}+K\right) \sqrt{\delta},
$$

что и требовалось.

Докажем (2.31). Если кривая Г спрямляема, то функция $\psi(z)$ имеет конечное полное изменение на $\gamma$. В силу теорем Рисса (см., например, [16; гл. 2, 3] или $[9 ;$ гл. 9,10$])$ отсюда следует абсолютная непрерывность функции $\psi(z)$ на $\gamma$ и наличие у ее производной $\psi^{\prime}(z)$ конечных угловых пределов почти всюду на $\gamma$. По граничной теореме единственности (см. там же) эти пределы отличны от нуля почти в каждой точке $t \in \gamma$. Так как $|C E(M)|>0$, то найдется $t \in C E(M)$ с этим свойством. Так как очевидно, что $\left|\psi^{\prime}(r t)\right| \leqslant \Pi_{1}=\Pi_{1}(\psi, t)$ при любом $r \in[0,1]$, то и (2.31) также доказано. 


\section{9. Две леммы, используемые в доказательстве теоремы 6.1.}

Лемма 2.9. Пусть $G$ - ограниченная односвязная область на $\mathbb{C}, \Gamma-$ открытая достижимая жорданова дуга ее границы $\partial G($ возможно, $\Gamma=\partial G)$, $\Gamma \in J(g, \varepsilon), w=\varphi(z)$ - некоторое однолистное конформное отображение области $G$ на единичный круг $D$. Тогда это отображение непрерывно продолжимо на $Г$ до гомеоморфизма $G \cup \Gamma$ на $D \cup \gamma(\gamma=\varphi(\Gamma))$, а затем непрерывно продолжимо с Г на $\bar{\Gamma}$ до гомеоморфизма $\bar{\Gamma}$ на $\bar{\gamma}$. Если при этом $R=R(\varphi, \Gamma)$ расстояние от точки $b:=\varphi^{-1}(0)$ до дуги $\Gamma$, а некоторое число $r \in(0, \varepsilon]$ удовлетворяет неравенству $9 g(r)<2 \pi R$, то

$$
\omega(\varphi, \bar{\Gamma}, \delta) \leqslant A_{0}(\varphi) \sqrt{g(\delta)}, \quad \delta \in[0, r] ; \quad A_{0}(\varphi)=3 K_{\Lambda} \sqrt{\left|\varphi^{\prime}(b)\right|},
$$

где $K_{\Lambda}$ - постоянная Лаврентьева из n. 2.3. Верно также и более общее неравенство

$$
\omega(\varphi, \bar{\Gamma}, \delta) \leqslant A_{1} \sqrt{g(\delta)}, \quad \delta \geqslant 0
$$

где конечная положительная величина $A_{1}$ не зависит от $\delta$.

ДокАЗАтЕльство. В соответствии с локальной формой теоремы Каратеодори (см. п. 2.2) непрерывно продолжим отображение $w=\varphi(z)$ с $G$ на $G \cup \Gamma$ до гомеоморфизма $G \cup \Gamma$ на $D \cup \gamma$, где $\gamma=\varphi(\Gamma)$, а затем непрерывно продолжим его с $\Gamma$ на $\bar{\Gamma}$ до гомеоморфизма $\bar{\Gamma}$ на $\bar{\gamma}$.

А) Докажем неравенство (2.32). Рассмотрим сначала случай $0 \in G, \varphi(0)=0$, $\varphi^{\prime}(0)=1$. Возьмем некоторую точку $t \in \bar{\Gamma}$ и положим $q:=9 /(2 \pi), 1<q<2$. Возьмем какое-либо $\delta \in(0, r]$ и некоторую точку $z \in \bar{\Gamma}$ при условии $|z-t| \leqslant \delta$. Тогда в силу неравенства $g(x) \geqslant x$ (см. п. 1.2) выполнено $\delta \leqslant g(\delta)<R / q$.

Обозначим через $D(t)=D(t, q g(\delta))$ открытый круг радиуса $q g(\delta)$ с центром в точке $t$. Так как $g(\delta)<R / q$, то точка нуль находится вне замыкания $\bar{D}(t)$ круга $D(t): 0 \in G \backslash \bar{D}(t)$. Пусть $\Delta=\Delta(t, \delta)$ обозначает ту связную компоненту открытого множества $G \cap D(t)$, в границу которой входит точка $t$ как точка рассматриваемой достижимой дуги $\bar{\Gamma}$. Отметим, что область $\Delta$ односвязна.

Для дальнейшего важно следующее утверждение: если $z \in \bar{\Gamma}$ и $|z-t| \leqslant \delta$, то $z \in \partial \Delta$.

В самом деле, так как $\Gamma \in J(g, \varepsilon)$, то в случае $\Gamma \neq \partial G$ величина диаметра дуги $L(t, z) \subset \bar{\Gamma}$ с концами $t$ и $z$ не превосходит $g(\delta)$, а в случае $\Gamma=\partial G$ величина диаметра хотя бы одной из двух дуг контура Г, имеющих своими концами точки $t$ и $z$ (обозначим эту дугу через $\Gamma(t, z)$ ), не превосходит $g(\delta)$, так как $\Gamma \in J(g, \varepsilon)$ и $|t-z| \leqslant \delta \leqslant \varepsilon$. Поэтому $\Gamma(t, z)$ вместе со своими концами лежит строго внутри открытого круга $D(t)$. Следовательно, точки $z$ и $t$ можно соединить некоторой кривой $\Lambda \subset G \cup\{t, z\}$, целиком лежащей внутри круга $D(t)$ и всеми своими неконцевыми точками лежащей внутри области $\Delta$. Отсюда следует, что $z \in \partial \Delta$, что и требовалось.

Продолжим доказательство неравенства (2.32). Очевидно, множество $G \cap$ $\partial D(t)$ распадается на конечное или счетное семейство открытых дуг (без общих точек), концы которых лежат на $\partial G$. Ровно одна из этих дуг (обозначим ее через $\lambda$ ) является общей граничной дугой для области $\Delta$ и той связной компоненты открытого множества $G \backslash \bar{D}(t)$, которая содержит точку нуль. Дуга $\lambda$ является разрезом области $G$ на следующие две односвязные области: 
1) содержащую точку нуль связную компоненту $G(0)$ открытого множества $G \backslash \lambda$;

2 ) содержащую область $\Delta$ внутри себя и точку $t$ (достижимую из $\Delta$ ) на своей границе - вторую связную компоненту $G(t)=G \backslash \overline{G(0)}$ множества $G \backslash \lambda$.

Отметим, что в границу области $G(t)$ входит всякая дуга $\smile t z \subset \bar{\Gamma}$, как только $|z-t| \leqslant \delta$. При этом очевидно, что $\operatorname{mes}_{1}(\lambda)<2 \pi q g(\delta)=9 g(\delta)$. По теореме Лаврентьева (п. 2.3) отсюда следует, что если $z \in \bar{\Gamma}$ и $|z-t| \leqslant \delta$, то $\rho_{\Lambda}(G ; z, t) \leqslant 9 g(\delta)$ и

$$
|\varphi(z)-\varphi(t)| \leqslant K_{\Lambda} \cdot 3 \sqrt{g(\delta)}
$$

$t, z \in \bar{\Gamma},|z-t| \leqslant \delta, 0<\delta \leqslant r(g, \varepsilon)$. Отсюда непосредственно следует неравенство $(2.32)$ при $\delta \in(0, r]$, а при $\delta=0$ оно очевидно.

В) Рассмотрим общий случай области $G$ и отображающей функции $\varphi$. При $b:=\varphi^{-1}(0)$ положим

$$
\begin{gathered}
k:=\varphi^{\prime}(b), \quad G_{1}:=\{\zeta=k \cdot(z-b): z \in G\}, \\
\Gamma_{1}:=\{\zeta=k \cdot(z-b): z \in \Gamma\} \subset \partial G_{1}, \quad g_{1}(x):=|k| g\left(\frac{x}{|k|}\right), \quad x \geqslant 0, \\
\varphi_{1}(\zeta):=\varphi\left(b+\frac{\zeta}{k}\right), \quad \zeta \in G_{1} \cup \Gamma_{1}, \quad R_{1}:=|k| R, \quad r_{1}=|k| r .
\end{gathered}
$$

Если теперь вместо $G, \Gamma, g(\delta), \varphi(z), R$ и $r$ взять $G_{1}, \Gamma_{1}, g_{1}(\delta), \varphi_{1}(\zeta), R_{1}$ и $r_{1}$ соответственно, то из неравенства $(2.34)$ получаем неравенство $(2.32)$ в общем случае:

$$
\begin{gathered}
\omega(\varphi, \bar{\Gamma}, \delta)=\omega\left(\varphi_{1}, \bar{\Gamma}_{1},|k| \delta\right) \leqslant 3 K_{\Lambda} \sqrt{g_{1}(|k| \delta)}=\left(3 K_{\Lambda} \sqrt{|k|}\right) \sqrt{g(\delta)}, \\
0 \leqslant \delta \leqslant r=r(g, R, \varepsilon) .
\end{gathered}
$$

C) Сейчас, заменив в $(2.32)$ величину $A_{0}$ некоторой бо́льшей величиной $A_{1}$, получим (2.33). Именно, так как $\varphi(\bar{\Gamma}) \subset \partial D$, то $|\varphi(z)-\varphi(t)| \leqslant 2$ при любых $z, t \in \bar{\Gamma}$, откуда $\omega(\varphi, \bar{\Gamma}, \delta) \leqslant 2, z, t \in \bar{\Gamma}$. Отсюда, из $(2.32)$ и неубывания функций $\omega(\varphi, \bar{\Gamma}, \delta)$ и $g(\delta)$ получаем неравенство $(2.33)$ в следующем виде:

$$
\omega(\varphi, \bar{\Gamma}, \delta) \leqslant \frac{2}{\omega(\varphi, \bar{\Gamma}, r(g, \varepsilon))} A_{0}(\varphi) \sqrt{g(\delta)}, \quad \delta \geqslant 0 .
$$

Следующая лемма представляет собой чисто геометрическое утверждение (как сказано выше, она понадобится в §6).

Лемма 2.10. Пусть $G$ - односвязная область на плоскости $\mathbb{C}, \Gamma$ - ограниченная открытая достижимая жорданова дуга ее гранищь $\partial G$, причем $\Gamma \neq \partial G$ u $\Gamma \in J(g, \eta)$ при некоторых $g(x)$ и $\eta>0$. Тогда для любой замкнутой дуги $\bar{\Sigma} \subset \Gamma$ (возможно, вырожденной в единственную точку) и любого компакта $E \subset G \cup \bar{\Sigma}$ найдутся такая открытая дуга $t^{\prime} t^{\prime \prime} \subset \Gamma$ с концами $t^{\prime}, t^{\prime \prime} \in \Gamma \backslash \bar{\Sigma}($ содержащая дугу $\bar{\Sigma}$ внутри себя) и такая односвязная область $G\left(t^{\prime}, t^{\prime \prime}\right) \subset G$ с жордановой гранищей $\Gamma\left(t^{\prime}, t^{\prime \prime}\right)$, что

1) $E \subset G\left(t^{\prime}, t^{\prime \prime}\right) \cup \bar{\Sigma}$;

2a) $\Gamma\left(t^{\prime}, t^{\prime \prime}\right) \cap \partial G=\smile t^{\prime} t^{\prime \prime}$;

2b) дуга $\Lambda=\Lambda\left(t^{\prime}, t^{\prime \prime}\right):=\Gamma\left(t^{\prime}, t^{\prime \prime}\right) \cap G$ с концами $t^{\prime}, t^{\prime \prime}$ является разрезом области $G$ на две односвязные ее подобласти $G\left(t^{\prime}, t^{\prime \prime}\right) u G_{0}:=G \backslash \overline{G\left(t^{\prime}, t^{\prime \prime}\right)}$, причем 
дважды гладкая замкнутая дуга $\bar{\Lambda}$ находится на положительном расстоянии как от рассматриваемого компакта $E$, так и от дуги $\bar{\Sigma}$;

3) $\Gamma\left(t^{\prime}, t^{\prime \prime}\right) \in J\left(g^{*}, \eta^{*}\right)$ nри $g^{*}(x)=g(x)+x\left(g^{*}(x) \leqslant 2 g(x)\right)$ и некотором $\eta^{*} \in(0, \eta]$.

ДокАЗАтельство. Разность $\Gamma \backslash \bar{\Sigma}$ распадается на две открытые жордановы дуги $\mu^{\prime}$ и $\mu^{\prime \prime}$; будем считать, что при движении по Г в положительном направлении обхода $G$ дуга $\mu^{\prime}$ предшествует дуге $\mu^{\prime \prime}$. Произведем возле дуги $\mu^{\prime}$ некоторые построения (построения возле дуги $\mu^{\prime \prime}$ аналогичны).

Возможны лишь два случая: А) имеется открытая дуга $\nu^{\prime} \subset \mu^{\prime}$, строго выпуклая во внешность области $G$; В) на $\mu^{\prime}$ такой дуги $\nu^{\prime}$ нет.

Случай А). Пусть $\tau(z)$ - величина угла с вершиной в точке $z \in \nu^{\prime}$, образованного направлением положительной полукасательной к кривой $\nu^{\prime}$ в этой точке (т.е. лучом, выходящим из $z$ касательно к кривой $\nu^{\prime}$ в этой точке в положительном направлении прохождения кривой $\left.\mu^{\prime}\right)$ с положительным направлением оси $O x$ (величина угла $\tau(z)$ отсчитана против часовой стрелки от направления $O x$ ). Заметим, что в силу спрямляемости дуга $\nu^{\prime}$ почти в каждой своей точке $z$ имеет обычную касательную; поэтому можем считать, что в концах дуги $\nu^{\prime}$ кривая $\mu^{\prime}$ имеет обычные касательные. Обозначим через $\Delta^{+}\left(\bar{\nu}^{\prime}\right)$ приращение величины угла $\tau(z)$ при прохождении точкой $z$ (в положительном направлении) дуги $\bar{\nu}^{\prime}$. При необходимости уменьшив дугу $\nu^{\prime}$ или выбрав другую дугу $\nu^{\prime} \subset \mu^{\prime}$ с касательными в ее концах, добьемся выполнения неравенства $\Delta^{+}\left(\bar{\nu}^{\prime}\right)<\pi / 8$.

Обозначим через $F^{\prime}$ множество всех точек на $\nu^{\prime}$, в которых у дуги $\nu^{\prime}$ имеются обычные касательные. Так как дуга $\nu^{\prime}$ выпукла, то у нее в каждой точке $t^{\prime}$ некоторого множества $F_{1}^{\prime} \subset F^{\prime}$ с $\operatorname{mes}_{1}\left(F_{1}^{\prime}\right)=\operatorname{mes}_{1}\left(F^{\prime}\right)$ существует парабола $P\left(\nu^{\prime}, t^{\prime}\right)$ не выше второй степени, имеющая с дугой $\nu^{\prime}$ в точке $t^{\prime}$ касание второго порядка (эта парабола изображает дифференциал 2-го порядка кривой $\nu^{\prime}$ в точке $t^{\prime}$; эта парабола может вырождаться в прямую). Парабола $P\left(\nu^{\prime}, t^{\prime}\right)$ имеет вершиной точку $t^{\prime}$, а осью - проходящую через $t^{\prime}$ перпендикулярно касательной к $\nu^{\prime}$ в точке $t^{\prime}$ прямую, и при этом вблизи $t^{\prime}$ парабола $P\left(\nu^{\prime}, t^{\prime}\right)$ выпукла в ту же сторону, что и $\nu^{\prime}$.

Парабола $P\left(\nu^{\prime}, t^{\prime}\right)$ может пересекаться с $\nu^{\prime}$ как угодно близко к $t^{\prime}$, что нежелательно. В таком случае мы заменим $P\left(\nu^{\prime}, t^{\prime}\right)$ похожей параболой $P^{*}\left(\nu^{\prime}, t^{\prime}\right)$, имеющей ту же вершину $t^{\prime}$ и ту же касательную в точке $t^{\prime}$, что и парабола $P\left(\nu^{\prime}, t^{\prime}\right)$ и кривая $\mu^{\prime}$, но уже не имеющей с $\nu^{\prime}$ вблизи $t^{\prime}$ общих точек, отличных от $t^{\prime}$. Очевидно, $P^{*}\left(\nu^{\prime}, t^{\prime}\right)$ уже не имеет касания 2-го порядка с $\nu^{\prime}$ в точке $t^{\prime}$.

Парабола $P^{*}\left(\nu^{\prime}, t^{\prime}\right)$ получается из $P\left(\nu^{\prime}, t^{\prime}\right)$ следующим образом. Введем декартову систему координат $O x^{\prime} y^{\prime}$ с началом в точке $t^{\prime}$ (в которой $x^{\prime}=0, y^{\prime}=0$ ), ось $O x^{\prime}$ направим по положительной полукасательной к $\nu^{\prime}$ в точке $t^{\prime}$, а ось $O y^{\prime}$ - по оси параболы $P\left(\nu^{\prime}, t^{\prime}\right)$ внутрь нее. После этого сделаем преобразование $x_{1}^{\prime}=x^{\prime}, y_{1}^{\prime}=y^{\prime}+a x^{\prime 2}, a>0$, плоскости $O x^{\prime} y^{\prime}$, переводящее параболу $P\left(\nu^{\prime}, t^{\prime}\right)$ в более "крутую" параболу $P^{*}\left(\nu^{\prime}, t^{\prime}\right)$. Здесь $a$ - какое-либо фиксированное положительное число, например, $a=1$. Это уже всегда настоящая парабола (а не прямая), не имеющая в некоторой окрестности точки $t^{\prime}$ других точек пересечения с $\mu^{\prime}$, кроме $t^{\prime}$. 
На правой ветви параболы $P^{*}\left(\nu^{\prime}, t^{\prime}\right)$, при взгляде на эту параболу изнутри нее, возьмем такую дугу $L^{\prime}$, что одним ее концом является точка $t^{\prime}$, а все остальные точки этой дуги, включая второй ее конец $z^{\prime}$, лежат в $G$.

Случай В). Рассмотрим ту же дугу $\mu^{\prime}$ в случае В). Читателя пусть не смущает то, что ниже в тексте встретятся обозначения, использованные при рассмотрении случая А) для обозначения совсем других объектов: это сделано специально - для сокращения заключительных рассуждений настоящего доказательства. Для дальнейшего напомним еще раз, что всегда $g(x) \geqslant x, x \geqslant 0$.

Так как в случае В) дуга $\mu^{\prime}$ не содержит поддуги, строго выпуклой наружу области $G$, то найдется открытый отрезок $J^{\prime} \subset \bar{G}$ со следующими свойствами:

I) $J^{\prime}$ находится на положительном расстоянии от $\partial G \backslash \mu^{\prime}$;

II) $J^{\prime}$ касается дуги $\mu^{\prime}$ одной своей стороной в некоторой своей внутренней точке $t^{\prime}$ (хотя бы одной);

III) в то же время с другой стороны этого отрезка можно построить некоторую односвязную жорданову область $G^{\prime} \subset G$ с границей $\partial G^{\prime}=J^{\prime} \cup \Gamma^{\prime}$, где жорданова дуга $\Gamma^{\prime}$ имеет своими концами концы отрезка $J^{\prime}$, всеми же своими неконцевыми точками $\Gamma^{\prime}$ лежит в $G \backslash J^{\prime}$.

Теперь символом $L^{\prime}$ обозначим некоторый отрезок $\left[t^{\prime}, z^{\prime}\right] \subset G^{\prime} \cup\left\{t^{\prime}\right\}$, перпендикулярный к отрезку $J^{\prime}$ в точке $t^{\prime}$.

Далее будем непрерывно двигать точку $z$ по дуге $\mu^{\prime}$ от точки $t^{\prime}$ в сторону дуги $\Sigma$, а возможно, и далее по дуге Г до тех пор, пока в первый раз случится какое-либо (хотя бы одно) из следующих трех событий В1), В2) или В3).

Событие В1): отрезок $\left[z^{\prime}, z\right]$, постоянно пересекая отрезок $J^{\prime}$ при указанном движении точки $z$ по дуге $\mu^{\prime}$ (от точки $t^{\prime}$ ), при некотором $z=z_{1}^{\prime}$ впервые пересек отрезок $J^{\prime}$ в каком-то его конце (при этом будет выполнено неравенство $\operatorname{diam} \smile t^{\prime} z_{1}^{\prime} \leqslant \eta$ (см. ниже В2)); очевидно, что тогда при любых $t \in\left[t^{\prime}, z^{\prime}\right]$ и $z \in \smile t^{\prime} z_{1}^{\prime}$ верны неравенства $\left|t-t^{\prime}\right| \leqslant|t-z| \leqslant g(|t-z|),\left|z-t^{\prime}\right| \leqslant|z-t| \leqslant g(|z-t|)$, и поэтому для кривой $L^{\prime}(z, t):=\left[t, t^{\prime}\right] \cup t^{\prime} z$ при указанном расположении точек $t$ и $z$ справедливы неравенства $\operatorname{diam}\left(L^{\prime}(z, t)\right) \leqslant\left|t-t^{\prime}\right|+\operatorname{diam}\left(\smile t^{\prime} z\right)$,

$$
\operatorname{diam}\left(L^{\prime}(z, t)\right) \leqslant|t-z|+g(|t-z|) \leqslant 2 g(|t-z|),
$$

$t \in L^{\prime}, z \in \smile t^{\prime} z_{1}^{\prime}$.

Событие В2): неравенство $\left|z-t^{\prime}\right|<\eta$ обратилось в равенство $\left|z-t^{\prime}\right|=\eta$ при некотором $z=z_{1}^{\prime}$; в этом случае неравенство (2.35) также выполняется для любых $t \in L^{\prime}$ и $z \in \smile t^{\prime} z_{1}^{\prime}$.

Событие В3): при указанном движении точки $z$ от точки $t^{\prime}$ до конца $b$ дуги Г не произошло ни событие В1), ни событие В2); тогда неравенство (2.35) выполняется для любых $t \in\left[t^{\prime}, z^{\prime}\right]$ и $z \in \smile t^{\prime} b$. Нетрудно видеть, что неравенство (2.35) верно также и в случае A), если под $L^{\prime}$ понимать указанную при рассмотрении случая А) дугу $\smile t^{\prime} z^{\prime}$ параболы $P^{*}\left(\nu^{\prime}, t^{\prime}\right)$.

Положим $\eta^{\prime}=\min \left\{\left|z^{\prime}-t^{\prime}\right|,\left|t^{\prime}-z_{1}^{\prime}\right|, \eta\right\}$ в случае В1), а в случаях В2), В3) и А) пусть $\eta^{\prime}=\min \left\{\left|z^{\prime}-t^{\prime}\right|, \eta\right\}$.

Далее, на $\mu^{\prime \prime}$ в случае, аналогичном А), выбираем дугу $\nu^{\prime \prime}$, точку $t^{\prime \prime} \in \nu^{\prime \prime}$, строим параболы $P\left(\nu^{\prime \prime}, t^{\prime \prime}\right)$ и $P^{*}\left(\nu^{\prime \prime}, t^{\prime \prime}\right)$, лишь дугу $L^{\prime \prime} \subset P^{*}\left(\nu^{\prime \prime}, t^{\prime \prime}\right)$ с началом $t^{\prime \prime}$ и концом $z^{\prime \prime}$ теперь возьмем на левой ветви параболы $P^{*}\left(\nu^{\prime \prime}, z^{\prime \prime}\right)$ (при взгляде изнутри нее). В случае же, аналогичном В), выбираем все (точку $t^{\prime \prime} \in \nu^{\prime \prime}$, отрезок $J^{\prime \prime}$, дугу $\Gamma^{\prime \prime}$ и область $G^{\prime \prime}$, отрезок $L^{\prime \prime}=\left[t^{\prime \prime}, z^{\prime \prime}\right]$ и т. д.) таким же 
образом, как выше были выбраны точка $t^{\prime} \in \nu^{\prime}$, отрезок $J^{\prime}$, дуга $\Gamma^{\prime}$ и область $G^{\prime}$, точка $z^{\prime \prime}$ и отрезок $L^{\prime}=\left[t^{\prime \prime}, z^{\prime \prime}\right]$ и т. д. (в случаях В) точка $z$ движется от $t^{\prime \prime}$ по дуге $\Gamma$ к ее началу $a)$. В каждом из случаев А), В1), В2) и В3) теперь верно неравенство (2.35) с заменой в нем значков "один штрих" значками "два штриха". Совершенно аналогично определению числа $\eta^{\prime}$ определяем число $\eta^{\prime \prime}$.

Очевидно, без ограничения общности можно считать, что $L^{\prime}$ и $L^{\prime \prime}$ не пересекаются ни между собой, ни с компактом $E$. Теперь проведем в области $G$ некоторую кривую $\Lambda_{1}$ от точки $z^{\prime \prime}$ к точке $z^{\prime}$ так, чтобы кривая $\Lambda:=L^{\prime \prime} \cup \Lambda_{1} \cup L_{-}^{\prime}$ ( $L_{-}^{\prime}$ обозначает кривую $L^{\prime}$, проходимую от точки $z^{\prime}$ к точке $\left.t^{\prime}\right)$ была дважды гладкой жордановой кривой и вместе с дугой $\smile t^{\prime} t^{\prime \prime} \subset \Gamma$ образовала замкнутую жорданову кривую $\Gamma(\Sigma) \supset \Sigma$, ограничивающую такую односвязную подобласть $G(\Sigma)$ области $G$, что $E \subset G(\Sigma) \cup \bar{\Sigma}$. Очевидно, кривая $\bar{\Lambda}$ находится на положительном расстоянии как от $E$, так и от $\bar{\Sigma}$ (как требуется в п. 2b) леммы).

Обозначим через $\delta(\Lambda)>0$ такое число, что $d(\Lambda ; \delta) \equiv \delta$ при любом $\delta \in(0, \delta(\Lambda)]$ (см. замечание к равенству (1.6)), и возьмем какое-либо положительное число

$$
\eta^{*} \leqslant \min \left\{\eta^{\prime}, \eta^{\prime \prime}, \delta(\Lambda)\right\} \leqslant \eta
$$

(последнее неравенство справедливо в силу того, что $\eta^{\prime}, \eta^{\prime \prime} \leqslant \eta$ ).

Возьмем какие-либо две точки $z, t \in \Gamma(\Sigma)$, для которых $|z-t| \leqslant \eta^{*}$. Тогда если $z, t \in \smile t^{\prime} t^{\prime \prime}$, то $\left.\operatorname{diam}(\smile z t) \leqslant g(|z-t|)\right)$. Если $z, t \in \Lambda$, то $\operatorname{diam}(\smile z t) \leqslant$ $|z-t| \leqslant g(|z-t|)$. Наконец, если одна из точек $t^{\prime}, t^{\prime \prime}$ является внутренней для дуги $\smile z t \subset \Gamma(\Sigma)$, то из $(2.35)$ получаем неравенство $\operatorname{diam}(\smile z t) \leqslant|z-t|+$ $g(|z-t|)$. Таким образом, $d(\Gamma(\Sigma) ; \delta) \leqslant \delta+g(\delta)=: g^{*}(\delta)$ при любом $\delta \leqslant \eta^{*}$, так что основная часть леммы 2.10 доказана. Остальные свойства кривой $\Gamma\left(t^{\prime}, t^{\prime \prime}\right)$ легко следуют из ее построения. Лемма 2.10 полностью доказана.

\section{§ 3. Отображения жордановых областей на круг}

Доказываемые ниже теоремы этой работы (формулируемые в обозначениях §1) всегда содержательны в том смысле, что правые части приводимых в них неравенств для модулей непрерывности отображающих функций всегда стремятся к нулю при $\delta \rightarrow 0$ (если выполнены условия рассматриваемой теоремы).

ТЕОрема 3.1. Пусть $G$-односвязная ограниченная область плоскости $\mathbb{C}$ с жордановой границей $\Gamma \in J(g, \varepsilon), w=\varphi(z)$ - однолистное конформное отображение этой области на единичный круг D. Тогда

$$
\omega(\varphi, \bar{G}, \delta) \leqslant A_{1} \sqrt{g(\delta)}, \quad \delta \geqslant 0,
$$

где $A_{1}$ не зависит от $\delta$.

Заметим, что при дополнительном предположении о том, что модуль непрерывности $\omega(\varphi, \partial G, \delta)$ функции $\varphi(z)$ на границе области $G$ имеет нормальную мажоранту в смысле Тамразова (см. [12]), эта теорема следует из леммы 2.9 и теоремы Тамразова (см. п. 2.4). Отметим, что, в частности, такой нормальной мажорантой является правая часть неравенства (2.33) с какой-либо постоянной $A_{1}$. В общем же случае эта теорема вытекает из приводимой ниже теоремы 3.2 (см. п. В) ее доказательства). 
Теорема 3.2. Пусть $G$ - односвязная ограниченная область плоскости $\mathbb{C}$ c границей $\Gamma \in J(g, \varepsilon)$, функиия $w=\varphi(z)$ однолистно и конформно отображает область $G$ на единичный круг $D, a R$ - расстояние от прообраза $b=\varphi^{-1}(0)$ точки $w=0$ до $\Gamma$, положительное же число $r \leqslant \varepsilon$ удовлетворяет условию $9 g(r)<2 \pi R$. Тогда

$$
\omega(\varphi, \bar{G}, \delta) \leqslant A_{0}(\varphi) \sqrt{g(\delta)}, \quad \delta \in[0, r], \quad A_{0}(\varphi)=3 K_{\Lambda} \sqrt{\left|\varphi^{\prime}(b)\right|},
$$

где $K_{\Lambda}$ - постоянная Лаврентьева (см. [10], а также $\left.n .2 .3\right)$.

ДоКАЗАТЕЛЬСтво. О гомеоморфной продолжимости отображения $w=\varphi(z)$ с $G$ на $G \cup \Gamma$ говорит теорема Каратеодори (см. введение).

A1) Докажем неравенство (3.2). Рассмотрим сначала случай $0 \in G, \varphi(0)=0$, $\varphi^{\prime}(0)=1$. Через $R$ обозначим расстояние от точки нуль до $\Gamma(R \geqslant 1 / 4$ по теореме Кёбе-Бибербаха). Положив $q:=9 /(2 \pi)(1<q<2)$, выберем число $r \in(0, \varepsilon]$ из условия $q g(r)<R$ (тогда $g(r)<R / q<R$ ) и фиксируем какое-либо $\delta \in[0, r]$. Так как $g(x) \geqslant x$ (см. п. 1.2 ), то $\delta \leqslant g(\delta)<R / q$.

Ниже в этом пункте точка $t \in \Gamma$ фиксирована, $D(t):=D(t, q g(\delta))$ обозначает открытый круг радиуса $q g(\delta)$ с центром в $t, \bar{D}(t)$ - замыкание $D(t)$. В силу сказанного $0 \in G \backslash \bar{D}(t)$.

Пусть $\Delta=\Delta(t, \delta)$ обозначает ту связную компоненту открытого множества $G \cap D(t)$, в границу которой входит точка $t$. Отметим, что область $\Delta$ односвязна.

Для дальнейшего нам потребуется следующее утверждение: если $z \in \bar{G}$ и $|z-t| \leqslant \delta$, то $z \in \bar{\Delta}$; в частности, если $z \in \Gamma$ и $|z-t| \leqslant \delta$, то $z \in \partial \Delta \cap \Gamma$. Докажем это. Итак, пусть $t, z \in \Gamma,|z-t| \leqslant \delta$. Из условия $\Gamma \in J(g, \varepsilon)$ следует (см. определение модуля колебания $d(L ; \delta)$ ), что диаметр хотя бы одной из дуг кривой $\Gamma$, имеющих своими концами точки $t$ и $z$, не превосходит $g(\delta)$. Таким образом, эта дуга (вместе со своими концами) лежит строго внутри открытого круга $D(t)$. Очевидно, точки $z$ и $t$ можно соединить некоторой кривой $\Lambda \subset$ $G \cup\{t, z\}$, проходящей вблизи Г и лежащей внутри $D(t)$. Поэтому $z \in \partial \Delta$.

Если $z \in G$, отрезок $[t, z]$ лежит в $G \cup\{t\}$, то требуемое следует из неравенства $|z-t| \leqslant \delta \leqslant g(\delta)$ и определения $\Delta$.

Если $z \in G,[t, z] \subset \bar{G}$, то в любой окрестности отрезка $[t, z]$ можно построить некоторую кривую $\Lambda \subset(G \cup\{t\}) \cap D(t)$, соединяющую $t$ с $z$ и поэтому со всеми своими точками, кроме $t \in \partial \Delta$, лежащую в области $\Delta$. В частности, $z \in \Delta$.

Если $z \in G$ и на $[t, z]$ имеются точки из дополнения к $\bar{G}$, то обозначим через $z^{*}$ точку на $[t, z] \cap \Gamma$, ближайшую к $z$. Так как $\left|z^{*}-t\right|<\delta$ и $\Gamma \in J(g, \varepsilon)$, то диаметр некоторой дуги $\Gamma\left(t, z^{*}\right) \subset \Gamma$ с концами $t$ и $z^{*}$ не превосходит $g(\delta)$, так что эта дуга лежит строго внутри $D(t)$. Поэтому можно построить некоторую кривую $\Lambda \subset(G \cup\{t\}) \cap D(t)$ с концами $t$ и $z$. Тогда $\Lambda \subset \Delta \cup\{t\}$, так что $z \in \Delta$.

Итак, доказано, что если $z \in \bar{G}$ и $|z-t| \leqslant \delta$, то $z \in \bar{\Delta}$.

Продолжим доказательство теоремы 3.2. Ясно, что множество $\partial D(t) \cap G$ распадается на конечное или счетное семейство открытых дуг без общих точек, концы которых лежат на Г. Ровно одна из этих дуг (обозначим ее через $\lambda$ ) является разрезом области $G$ на две непересекающиеся односвязные области (для которых $\lambda$ является общей граничной дугой): на область $G(0)$, содержащую точку нуль внутри себя, и область $G(t)$, включающую в себя область $\Delta$ и имеющую точку $t$ своей достижимой (из $G(t)$ и из $\Delta$ ) точкой своей границы. 
Отметим, что $G(t)$, как и $\Delta$, содержит множество $G \cap D(t, \delta)(D(t, \delta)$ - круг радиуса $\delta$ с центром в точке $t$ ) внутри себя и что $t$ входит в границу области $G(t)$ вместе с некоторой замкнутой достижимой из $G(t)$ поддугой дуги Г (а именно, со связной компонентой множества $\Gamma \cap \bar{D}(t, \delta))$ на жордановом контуре $\partial \Delta$.

Очевидно, $\operatorname{mes}_{1}(\lambda)<2 \pi q g(\delta)=9 g(\delta)$. В обозначениях теоремы Лаврентьева и леммы 2.9 отсюда следует, что если $z \in \bar{G},|z-t| \leqslant \delta$, то $\rho(G ; z, t) \leqslant 9 g(\delta)$ и

$$
|\varphi(z)-\varphi(t)| \leqslant K_{\Lambda} \cdot 3 \sqrt{g(\delta)}, \quad t \in \Gamma, \quad z \in \bar{G}, \quad|z-t| \leqslant \delta \leqslant r .
$$

А2) Итак, при условиях п. А1) неравенство (3.3) доказано для любых $t \in \Gamma$ и $z \in \bar{G}$ при условии $|z-t| \leqslant \delta, \delta \in(0, r]$ (при $\delta=0$ это неравенство очевидно).

Покажем, что это неравенство верно для любых точек $t, z \in \bar{G}$ при том же условии $|z-t| \leqslant \delta \leqslant r$ (напомним, что случай, когда хотя бы одна из этих точек лежит на $\Gamma$, уже рассмотрен выше в п. А1)).

Пусть $z^{\prime}, z^{\prime \prime} \in G$. Обозначим через $G\left(z^{\prime}, z^{\prime \prime}\right)$ связную компоненту открытого множества $\left\{z: z \in G, z+\left(z^{\prime \prime}-z^{\prime}\right) \in G\right\}$, содержащую точку $z^{\prime}$ (очевидно, это множество содержит точку $z^{\prime}$ с некоторой ее окрестностью). В силу принципа максимума модуля для аналитических функций при $z \in \overline{G\left(z^{\prime}, z^{\prime \prime}\right)}$ функция $f(z)=\varphi\left(z+\left(z^{\prime \prime}-z^{\prime}\right)\right)-\varphi(z)$ достигает максимума своего модуля лишь на $\partial G\left(z^{\prime}, z^{\prime \prime}\right)$. Пусть этот максимум достигается в некоторой точке $\zeta \in \partial G\left(z^{\prime}, z^{\prime \prime}\right)$. Последнее означает, что хотя бы одна из точек $\zeta, \zeta+\left(z^{\prime \prime}-z^{\prime}\right)$ принадлежит границе области $G$. Отсюда следует, что

$$
\left|\varphi\left(z^{\prime \prime}\right)-\varphi\left(z^{\prime}\right)\right|=\left|\varphi\left(z^{\prime}+\left(z^{\prime \prime}-z^{\prime}\right)\right)-\varphi\left(z^{\prime}\right)\right|<\left|\varphi\left(\zeta+\left(z^{\prime \prime}-z^{\prime}\right)\right)-\varphi(\zeta)\right|,
$$

где хотя бы одна из точек $\zeta, \zeta+\left(z^{\prime \prime}-z^{\prime}\right) \in \bar{G}$ лежит на $\partial G$. Отсюда и из $(3.3)$ следует, что если $z^{\prime}, z^{\prime \prime} \in \bar{G},\left|z^{\prime \prime}-z^{\prime}\right| \leqslant \delta \leqslant r$, то $\left|\varphi\left(z^{\prime \prime}\right)-\varphi\left(z^{\prime}\right)\right| \leqslant 3 K_{\Lambda} \sqrt{g(\delta)}$. Таким образом, неравенство (3.2) при принятых в п. А1) дополнительных условиях имеет следующий вид:

$$
\omega(\varphi, \bar{G}, \delta) \leqslant 3 K_{\Lambda} \sqrt{g(\delta)}, \quad \delta \in[0, r]
$$

$K_{\Lambda}$ - абсолютная постоянная из теоремы Лаврентьева (п. 2.3). Вспомним эти дополнительные условия: $0 \in G, \varphi(0)=0, \varphi^{\prime}(0)=1$. Сейчас мы от них освободимся.

А3) При $b:=\varphi^{-1}(0)$ положим

$$
\begin{gathered}
k:=\varphi^{\prime}(b), \\
G_{1}:=\{\zeta=k \cdot(z-b): z \in G\}, \quad \Gamma_{1}:=\partial G_{1}=\{\zeta=k \cdot(z-b): z \in \Gamma\}, \\
g_{1}(x):=|k| g\left(\frac{x}{|k|}\right), \quad \varphi_{1}(\zeta):=\varphi\left(b+\frac{\zeta}{k}\right), \quad \zeta \in \bar{G}_{1}, \\
R_{1}:=|k| R, \quad r_{1}=|k| r .
\end{gathered}
$$

Если вместо $G, \Gamma, g(\delta), \varphi(z), R$ и $r$ взять соответственно $G_{1}, \Gamma_{1}, g_{1}(\delta), \varphi_{1}(\zeta)$, $R_{1}$ и $r_{1}$, то из равенства $\omega(\varphi, \bar{G}, \delta)=\omega\left(\varphi_{1}, \bar{G}_{1},|k| \delta\right)$ и неравенства $(3.4)$ получаем неравенство (3.2) в общем случае:

$$
\omega(\varphi, \bar{G}, \delta) \leqslant 3 K_{\Lambda} \sqrt{g_{1}(|k| \delta)}=\left(3 K_{\Lambda} \sqrt{|k|}\right) \sqrt{g(\delta)}, \quad 0 \leqslant \delta \leqslant r .
$$


В) Теперь, заменив в $(3.2)$ величину $A_{0}(\varphi)$ некоторой большей величиной $A_{1}$, получим (3.1). Для этого заметим, что $|\varphi(z)-\varphi(t)| \leqslant 2$ для любой пары точек $z, t \in \bar{G}$. Поэтому $\omega(\varphi, \bar{G}, \delta) \leqslant 2, \delta \geqslant 0$. Отсюда, из $(3.5)$ и неубывания функций $\omega(\varphi, \bar{G}, \delta)$ и $g(\delta)$ при $\delta \geqslant 0$ получаем неравенство (3.1) в следующем виде:

$$
\omega(\varphi, \bar{G}, \delta) \leqslant \frac{2}{\omega(\varphi, \bar{G}, r)}\left(3 K_{\Lambda} \sqrt{|k|}\right) \sqrt{g(\delta)}, \quad \delta \geqslant 0 .
$$

Доказательство неравенств (3.1) и (3.2) закончено.

СЛЕДСТВИЕ 3.1. Если граница Г ограниченной жордановой области $G$ принадлежит классу $J(g, \varepsilon)$ с $g(x) \leqslant c x^{k}, x \in[0, \varepsilon]$, где $\varepsilon>0, c>0 u k \in(0,1]-$ некоторые постоянные, то для модуля непрерывности любого однолистного конформного отображсения $w=\varphi(z)$ области $G$ на единичный круг $D$ выполнено неравенство

$$
\omega(\varphi, \bar{G}, \delta) \leqslant A \delta^{k / 2}, \quad \delta \geqslant 0,
$$

где $A$ не зависит от $\delta$.

ЗАмЕчАниЕ 3.1. Пусть гладкая открытая жорданова дуга (или контур) Г является ляпуновской или хотя бы обобщенно ляпуновской. Последнее означает, что модуль непрерывности $\omega(\delta)$ какой-либо непрерывной ветви $\vartheta(s)$ угла положительной касательной к $\Gamma$ в точках $z=z(s)$ (таково натуральное уравнение кривой $\Gamma, s \in(0, S:=|\Gamma|))$ с положительным направлением действительной оси удовлетворяет (как функция параметра $s$ ) условию Дини

$$
\int_{0}^{S} \frac{\omega(r)}{r} d r<\infty
$$

Если $\omega(\delta)=o\left(\delta^{\nu}\right)$ при некотором $\nu>0$, то $\Gamma$ называют ляпуновской кривой или кривой Ляпунова. Известно (см. [3]), что в случае открытой достижимой обобщенно ляпуновской дуги Г границы ограниченной односвязной области $G$ всякое однолистное конформное отображение $w=\varphi(z)$ области $G$ на круг $D$ и обратное к нему отображение $z=\psi(w)$ непрерывно дифференцируемы на $G \cup \Gamma$ и на $D \cup \gamma, \gamma=\varphi(\Gamma)$, соответственно, причем $\varphi^{\prime}(z) \neq 0$ на $G \cup \Gamma, \psi^{\prime}(w) \neq 0$ на $D \cup \gamma$.

\section{§4. Отображения круга на жордановы области}

Ниже используются обозначения из $\S 1$, а также следующие обозначения и неравенства из леммы 2.7: если $t \in C$ и $\pi S_{t}(r) \leqslant u\left(l_{t}^{2}(r)\right), r \in[0, \rho]$, то $F(r)=$ $F(\psi, t ; r) \leqslant U^{-1}\left(a ; \log \left(E_{t}(\rho) r\right)\right), r \in(0, \rho]$, где

$$
E_{t}(\rho):=\rho^{-1} \exp \{U(a ; F(\psi, t ; \rho))\} \leqslant \rho^{-1} \exp \left\{U\left(a ; \pi S_{t}(\rho)\right)\right\}
$$

(см. $(2.26)$ и $(2.28)) ; E_{t}(\rho)=E(\psi, \gamma, t, u, a ; \rho) ;$ если $\bar{\sigma}$ - такая замкнутая поддуга дуги $\gamma$, что $\bar{\sigma}_{t}(\rho) \subset \gamma, t \in \bar{\sigma}$, где $\rho \in(0,2)$ не зависит от $t \in \bar{\sigma}$, то положим

$$
E_{\sigma}(\rho):=\sup \left\{E_{t}(\rho): t \in \bar{\sigma}\right\}
$$

$E_{\sigma}(\rho) \leqslant \rho^{-1} \exp \left\{U\left(a ; \pi \sup \left\{S_{t}(\rho): t \in \bar{\sigma}\right\}\right)\right\} \leqslant \rho^{-1} \exp \left\{U\left(a ; \pi, \operatorname{mes}_{2}(G)\right)\right\}$. 
В этих обозначениях справедлива следующая теорема.

Теорема 4.1. Пусть $w=\psi(z)$ - однолистное конформное отображение единичного круга $D$ на односвязную область $G \subset \mathbb{C}, \Gamma$ - ограниченная открытая достижимая жорданова дуга гранищъ $\partial G$ этой области (возможно, $\Gamma=\partial G), \Gamma \in J(g, \varepsilon)$, точка $t \in \gamma:=\psi^{-1}(\Gamma)$ фиксирована, число $\rho \in(0,2)$ таково, что $\overline{\sigma_{t}(\rho)} \subset \gamma$ u $\operatorname{diam}\left(\Sigma_{t}(\rho)\right) \leqslant \varepsilon$. Если при этом функиия $u(x) \geqslant 0$ определена и строго возрастает при $x \geqslant 0$ (она может быть разрывной), $u(0)=u(0+)=0, u(x) \geqslant x, x \geqslant 0, u$ выполнено условие

$$
\pi S_{t}(r) \leqslant u\left(l_{t}^{2}(r)\right), \quad r \in(0, \rho]
$$

то при любом $z \in \overline{\Delta_{t}(\rho / e)}($ см. п. 1.4) справедливо неравенство

$$
\begin{aligned}
|\psi(z)-\psi(t)| & \leqslant 2 g\left(\sqrt{U^{-1}\left(a ; \log \left(E_{t}(\rho) e|z-t|\right)\right)}\right) \\
& \left.=2 g\left(\sqrt{U^{-1}\left(a ; U(a ; F(\rho))+1+\log \left(\frac{|z-t|}{\rho}\right)\right.}\right)\right) .
\end{aligned}
$$

Если при этом открытая дуга $\sigma$ содержится в $\gamma$ вместе со своим замыканием $\bar{\sigma}$ (допускается случай $\sigma=\gamma=C$, т.е. $\Gamma=\partial G)$ и для некоторого числа $\rho=$ const $>0$ выполнень все условия теоремы (включая и (4.3)) для каждой точки $t \in \bar{\sigma}$, то для любых точек $t \in \bar{\sigma} u z \in D \cup \gamma$ при условии $|z-t| \leqslant \rho /$ е верно неравенство

$$
|\psi(z)-\psi(t)| \leqslant 2 g\left(\sqrt{U^{-1}\left(a ; \log \left(E_{\sigma}(\rho) e|z-t|\right)\right)}\right) .
$$

ЗАмЕчАнИЕ 4.1. Правые части неравенств (4.4) и (4.5) не зависят от выбора числа $a>0$, входящего в определения $U(a ; x)$ (см. (1.6)), $U^{-1}(a ; y), E_{t}(\rho)=$ $\rho^{-1} \exp \{U(a ; F(\rho))\}$ и $E_{\sigma}(\rho)$. Поэтому в ряде случаев ниже можно считать $a=1$.

ЗАмЕЧАНИЕ 4.2. Если при условиях теоремы 4.1 дуга Г является гладкой обобщенно ляпуновской (см. замечание 3.1 ), то $\psi(z)$ непрерывно дифференцируема на $D \cup \gamma$, а $w=\psi^{-1}(z)$ непрерывно дифференцируема на $G \cup \Gamma$.

ЗАмЕЧАНИЕ 4.3. Отметим, что при любых допустимых $u(x)$ (см. $\S 1)$ имеем (см. (1.6)) соотношения $y=u(x) \geqslant x, x=u^{-1}(y) \leqslant y, 1 / u^{-1}(y) \geqslant 1 / y$; при $a=1$ и $0<X \leqslant 1$ верны также соотношения $-Y=-U(1 ; X) \geqslant \log (1 / X)$ и $Y=U(1 ; X) \leqslant \log X, X=U^{-1}(1 ; Y) \geqslant e^{Y}$. Далее, поскольку $g(x) \geqslant x$, то $g\left(\sqrt{U^{-1}(1 ; \log \delta)}\right) \geqslant \sqrt{\delta}$. Итак, при любых допустимых функциях $u(x)$ и $g(x)$ верно неравенство

$$
g\left(\sqrt{U^{-1}(1 ; \log \delta)}\right) \geqslant \sqrt{\delta}, \quad \delta \in[0,1] .
$$

Это неравенство нам понадобится в $§ 6$ при доказательстве теоремы 6.2.

ДОКАЗАТЕЛЬСТВо тЕОРЕМЫ 4.1. Если $0<r \leqslant \rho, z \in \bar{D}$ и $|z-t|=r / e$, то из второй части леммы 2.7 (см. $(2.27))$ и неравенства $g(x) \geqslant x$ (см. п. 1.2) получаем для некоторого числа $\widehat{r}=\widehat{r}(\psi, t ; r) \in[r / e, r]$ включение $z \in \Delta_{t}(\widehat{r})$ и 
неравенства

$$
\begin{aligned}
&|\psi(z)-\psi(t)| \leqslant \operatorname{diam}\left(\psi\left(\Delta_{t}(\widehat{r})\right)\right) \leqslant \operatorname{diam}\left(\Lambda_{t}(\widehat{r})\right)+\operatorname{diam}\left(\Sigma_{t}(\widehat{r})\right) \\
& \leqslant l_{t}(\widehat{r})+g\left(l_{t}(\widehat{r})\right) \leqslant 2 g\left(l_{t}(\widehat{r})\right) \leqslant 2 g\left(\sqrt{U^{-1}\left(a ; \log \left(E_{t}(\rho) r\right)\right)}\right), \\
&|\psi(z)-\psi(t)| \leqslant 2 g\left(\sqrt{U^{-1}\left(a ; \log \left(E_{t}(\rho) r\right)\right)}\right) .
\end{aligned}
$$

Так как $r=e|z-t|$, то (4.4) доказано, а из него непосредственно следует (4.5).

Независимость правых частей неравенств (4.4) и (4.5) от выбора числа $a$ следует из леммы 2.7 (также и из леммы 2.6). Теорема 4.1 доказана полностью.

Сейчас мы рассмотрим некоторые частные случаи функций $g, u$ и докажем интересующие нас следствия теоремы 4.1. Будем придерживаться обозначений и определений $\S 1$.

Пусть $Г$ - ограниченная открытая достижимая жорданова дуга границы области $G \subset \mathbb{C}, \Gamma \in J(g, \varepsilon)$ (напомним, что $J(g)=J(g,+\infty)$ и $J_{0}(g)=J_{0}(g,+\infty)$ ), функция $w=\psi(z)$ однолистно и конформно отображает единичный круг $D$ на область $G, t \in \gamma=\psi^{-1}(\Gamma)$. Тогда если при некотором $r \in(0, \bar{\rho}]$, где $\bar{\rho}=\bar{\rho}(\psi, \Gamma, t, \varepsilon)$ (см. п. 1.4) выполнено включение $\Sigma_{t}(r) \subset \Gamma$, то по (2.6) (лемме 2.1) при $\gamma_{2}:=\Sigma_{t}(r), \gamma_{1}:=\Lambda_{t}(r)$ и $S_{t}(r):=\operatorname{mes}_{2}\left(\psi\left(\Delta_{t}(r)\right)\right)$ получаем неравенство

$$
\pi S_{t}(r) \leqslant d_{g}\left(l_{t}^{2}(r)\right), \quad r \in(0, \bar{\rho}], \quad d_{g}(x):=\frac{\pi^{2}}{4} g^{2}(\sqrt{x})+\frac{x}{2} .
$$

Так же, как выше (см. (1.6)) по функции $u(x)$ мы построили $U(a ; x)$, по функциям $d_{g}(x)$ строим $D_{g}(a ; x)$ :

$$
d_{g}(x):=\frac{\pi^{2}}{4} g^{2}(\sqrt{x})+\frac{x}{2}, \quad D_{g}(a ; x):=\int_{a}^{x} \frac{d y}{d_{g}^{-1}(y)} .
$$

При $\Gamma \in J_{0}(g, \varepsilon)$ по (2.6) (лемма 2.1) аналогично получаем

$$
\begin{gathered}
\pi S_{t}(r) \leqslant m_{g}\left(l_{t}^{2}(r)\right), \quad r \in\left(0, \bar{\rho}_{0}\right], \\
m_{g}(x):=\frac{1}{4}(g(\sqrt{x})+\sqrt{x})^{2}, \quad M_{g}(a ; x):=\int_{a}^{x} \frac{d y}{m_{g}^{-1}(y)} .
\end{gathered}
$$

При $\Gamma \in J_{0}(g)$ с $g(x) \leqslant c x$ по лемме 2.4 получаем $\pi S_{t}(r) \leqslant S(c) l_{t}^{2}(r)$ при всех $r \in\left(0, \bar{\rho}_{0}\right]$ и

$$
\begin{gathered}
y=u(x)=m m_{c}(x):=\pi S(c) x, \quad m m_{c}^{-1}(y)=\frac{y}{\pi S(c)}, \\
M M_{c}(a ; x):=\int_{a}^{x} \frac{d y}{y /(\pi S(c))}=\pi S(c) \log \left(\frac{x}{a}\right), \quad y=M M_{c}^{-1}(1 ; y)=a e^{y /(\pi S(c))} .
\end{gathered}
$$

В силу сказанного в $\S 1$ о функции $U^{-1}(a ; y)$ функции $D_{g}^{-1}(a ; y), M_{g}^{-1}(a ; y)$, $M M_{c}^{-1}(a ; y)$, определенные равенствами (4.8), (4.10) и (4.12), при $y \in(-\infty,+\infty)$ непрерывно строго возрастают, положительны и выпуклы вниз, стремятся к нулю при $y \rightarrow-\infty$ и стремятся к $+\infty$ при $y \rightarrow+\infty$. 
Теорема 4.2. Пусть $w=\psi(z)$ - однолистное конформное отображение единичного круга $D$ на ограниченную область $G$ с жордановой гранищей $\Gamma \in$ $J(g, \varepsilon)$. Тогда

$$
\omega(\psi, \bar{D}, \delta) \leqslant B g\left(\sqrt{D_{g}^{-1}(\log \delta)}\right), \quad \delta \geqslant 0,
$$

где $B=2+4\left(1+E_{\partial D}(\bar{\rho})\right) e / \bar{\rho}$ не зависит от $\delta($ см. $(2.26))$.

ЗАмЕЧАНИЕ 4.4. Отметим, что для любой допустимой функции $g(x)$ (см. п. 1.2) при $u(x)=d_{g}(x)$ (см. (4.8)) общее неравенство (4.6) можно существенно усилить. Именно, так как $g(x) \geqslant x$, то

$$
\begin{gathered}
y=d_{g}(x) \geqslant \frac{\left(\pi^{2}+2\right) x}{4}, \quad x=d_{g}^{-1}(y) \leqslant \frac{4 y}{\pi^{2}+2}, \quad \frac{1}{d_{g}^{-1}(y)} \geqslant \frac{4^{-1}\left(\pi^{2}+2\right)}{y}, \\
\int_{X}^{1} \frac{1}{d_{g}^{-1}(y)} d y \geqslant \int_{X}^{1} \frac{4^{-1}\left(\pi^{2}+2\right)}{y} d y, \quad 0<X \leqslant 1, \\
-Y=-D_{g}(1 ; X) \geqslant 4^{-1}\left(\pi^{2}+2\right) \log \frac{1}{X}, \quad Y=D_{g}(1 ; X) \leqslant 4^{-1}\left(\pi^{2}+2\right) \log X, \\
0<X \leqslant 1, \\
X=D_{g}^{-1}(Y) \geqslant \exp \left\{\frac{4 Y}{\pi^{2}+2}\right\} .
\end{gathered}
$$

Так как $g(x) \geqslant x$, то отсюда $g\left(\sqrt{D_{g}^{-1}(1 ; \log \delta)}\right) \geqslant \delta^{k}$ при $k=2 /\left(\pi^{2}+2\right)$ и любых $\delta \in[0,1]$. Итак, всегда

$$
g\left(\sqrt{D_{g}^{-1}(1 ; \log \delta)}\right) \geqslant \delta^{k}, \quad \delta \in[0,1], \quad k=\frac{2}{\pi^{2}+2} \approx \frac{1}{6}
$$

(сравните со следствием 4.1). Для нужд замечания 6.3 отметим, что, как это легко увидеть из только что приведенных выкладок, в случае $g(x) \equiv x, x \in$ $[0,1]$, верно равенство

$$
g\left(\sqrt{D_{g}^{-1}(1 ; \log \delta)}\right)=\delta^{k}, \quad \delta \in[0,1], \quad k=\frac{2}{\pi^{2}+2} .
$$

\section{ДОКАЗАТЕЛЬСТВО ТЕОРЕМЫ 4.2.}

1) Прежде всего отметим, что при значении $\bar{\rho}$, выбранном в условии теоремы 4.2 , в случае равенства $u(x) \equiv d_{g}(x)$ выполнены условия теоремы 4.1 . Следовательно, для каждой пары точек $t \in \partial D$ и $z \in \bar{D}$, для которой $|z-t| \leqslant$ $\delta \leqslant \bar{\rho} / e$, имеем неравенство

$$
|\psi(z)-\psi(t)| \leqslant 2 g\left(\sqrt{D_{g}^{-1}\left(a ; \log \left(E_{\partial D}(\bar{\rho}) e \delta\right)\right)}\right) .
$$

2) Возьмем некоторое $h \in D$ и рассмотрим на замкнутой круговой луночке $\Delta(h):=\{z:|z| \leqslant 1,|z+h| \leqslant 1\}$ функцию $f(z)=\psi(z)-\psi(z+h)$. В силу принципа максимума модуля $|f(z)|$ достигает максимума на $\Delta(h)$ в некоторой точке $\zeta \in \partial \Delta(h)$. Это означает, что хотя бы одна из точек $z=\zeta$ или $t=\zeta+h$ лежит на границе $C$ круга $D$. Поэтому при $0 \leqslant \delta \leqslant \bar{\rho} / e$ и $|h| \leqslant \delta$ получаем из (4.16) неравенство

$$
\omega(\delta):=\omega(\psi, \bar{D}, \delta) \leqslant 2 g\left(\sqrt{D_{g}^{-1}\left(a ; \log \left(E_{\partial D}(\bar{\rho}) e \delta\right)\right)}\right), \quad 0 \leqslant \delta \leqslant \frac{\bar{\rho}}{e}
$$


$3)$ Обобщим (4.17) на все $\delta \geqslant 0$. Пусть сначала $0<\delta \leqslant 2$. Если $E_{\partial D}(\bar{\rho}) \geqslant 1$, то при $\lambda:=2 E_{\partial D}(\bar{\rho}) e / \bar{\rho}$ получаем $\delta / \lambda=\delta \bar{\rho} /\left(2 E_{\partial D}(\bar{\rho}) e\right) \leqslant \bar{\rho} / e$. Следовательно, в этом случае неравенство (4.17) остается в силе, если в нем $\delta$ заменить на $\delta / \lambda$ (при условии $0 \leqslant \delta \leqslant 2$ ). При этом под знаком логарифма справа в $(4.17)$ окажется выражение

$$
E_{\partial D}(\bar{\rho}) e \cdot \frac{\delta}{\lambda}=E_{\partial D}(\bar{\rho}) e \cdot \frac{\delta \bar{\rho}}{2 e E_{\partial D}(\bar{\rho})}=\frac{\delta \bar{\rho}}{2} \leqslant \delta
$$

(напомним, что $0<\bar{\rho} \leqslant 2$ ). Учитывая все это, из $(2.2)$ и (4.17) получаем неравенство

$$
\omega(\delta) \leqslant(1+\lambda) \omega\left(\frac{\delta}{\lambda}\right) \leqslant\left(1+2 E_{\partial D} \frac{(\bar{\rho}) e}{\bar{\rho}}\right) \cdot 2 g\left(\sqrt{D_{g}^{-1}(\log \delta)}\right) .
$$

Пусть теперь $E_{\partial D}(\bar{\rho})<1$ и по-прежнему $0<\delta \leqslant 2$. Так как $\delta \bar{\rho} /(2 e) \leqslant \bar{\rho} / e$, то, полагая $\lambda:=2 e / \bar{\rho}$, аналогично предыдущему получаем неравенство для $\omega(\delta)$, которое выглядит как неравенство (4.18), в котором выражение $E_{\partial D}(\bar{\rho})$ заменено единицей. Это вместе с (4.18) доказывает справедливость неравенства (4.13) при всех $\delta \in[0,2]$.

Наконец, пусть $\delta \geqslant 2$. Так как $\omega(\delta)=\omega(2)$ при всех $\delta \geqslant 2$, а правая часть неравенства (4.13) возрастает с ростом $\delta$, то неравенство (4.13) и теорема 4.2 доказаны.

Заметим, что теорему 4.2 (с другим коэффициентом $B$ ) можно получить также и из оценки для $\omega(\psi, C, \delta)$ теоремы 4.1 , пользуясь неравенством Тамразова (2.4).

СлеДСтвиЕ 4.1. Если в условиях теоремы 4.2 имеем $\partial G \in J(g, \varepsilon)$ с $g(x) \leqslant$ $c x^{k}$ при $0<x \leqslant \varepsilon$, где $\varepsilon>0, c>1 u k \in(0,1]$ постояннь, то при $0 \leqslant \delta \leqslant 2$ справедливи следующие неравенства:

a) $\omega(\psi, \bar{D}, \delta) \leqslant A \delta^{n(c)}$ c $n(c)=2 /\left(\pi^{2} c^{2}+2\right)$ npu $k=1$;

b) $\omega(\psi, \bar{D}, \delta) \leqslant N /(1+|\log \delta|)^{\nu(k)}$ c $\nu(k)=k^{2} /(2-2 k)$ nрu $0<k<1$. Здесъ положительные константы $А$ и $N$ не зависят от $\delta$.

Легко установить, что при $k=1$ и любом $c \geqslant 1$ верно неравенство $n(c) \leqslant$ $n(1)<0.1685$. Обратно, при $1 \leqslant c<\sqrt{10} / \pi$ также просто получаем, что $n(c)>1 / 6=0.1666 \ldots$ (сравните с замечанием 4.4).

ДОКАЗАТЕЛЬСТВО СЛЕДСТВИЯ 4.1. Пусть $g(x) \leqslant c x^{k}$, где $c \geqslant 1$ и $0<k \leqslant 1$. Если $0 \leqslant x \leqslant 1$, то в силу (4.7) имеем $y=d_{g}(x) \leqslant A x^{k}$ с $A:=\left(\pi^{2} c^{2}+2\right) / 4$, откуда

$$
x=d_{g}^{-1}(y) \geqslant\left(\frac{y}{A}\right)^{1 / k}, \quad \frac{1}{d_{g}^{-1}(y)} \leqslant\left(\frac{A}{y}\right)^{1 / k} .
$$

Если $k=1$, то в силу (4.8) при $y:=D_{g}(a ; x) \geqslant A \log (x / a), 0<x \leqslant a$, $x=D_{g}^{-1}(a ; y) \leqslant a \exp \{y / A\}$. Из неравенства (4.13) теоремы 4.2 получаем при $0 \leqslant \delta \leqslant 1($ с $B=B(\psi, a))$, что

$$
\omega(\psi, \bar{D}, \delta) \leqslant B c \sqrt{a \exp \left\{\frac{\log \delta}{A}\right\}} \leqslant B c \sqrt{a} \delta^{n(c)}, \quad n(c)=\frac{2}{\pi^{2} c^{2}+2} .
$$


Если $0<k<1$, то из (4.8) получаем при $0<x \leqslant a<1$ :

$$
\begin{gathered}
y=D_{g}(a ; x)>B-A^{1 / k} \frac{k}{1-k} x^{1-1 / k}, \quad B=A^{1 / k} \frac{k}{1-k} a^{1-1 / k}, \\
B-y<A^{1 / k} \frac{k}{1-k} x^{1-1 / k}, \quad A^{-1 / k} \frac{1-k}{k} x^{(1-k) / k}<\frac{1}{B-y} \\
D_{g}^{-1}(a ; y)=x<H(A, k)(B-y)^{-k /(1-k)}
\end{gathered}
$$

где $\left.H(A, k):=A^{1 /(1-k)}(k / 1-k)\right)^{k /(1-k)}$.

Отсюда и из теоремы 4.2 получаем неравенство

$$
\omega(\psi, \bar{D}, \delta) \leqslant N_{1}(B-\log \delta)^{-\nu}=N_{1}(B+|\log \delta|)^{-\nu}, \quad \nu(k)=\frac{k^{2}}{2-2 k},
$$

где $0 \leqslant \delta \leqslant 1$, а $N_{1}$ не зависит от $\delta$. Так как $\omega(\psi, \bar{D}, \delta) \leqslant 2 \omega(\psi, \bar{D}, \delta / 2)$, то при $0 \leqslant \delta \leqslant 2$ отсюда получаем неравенство

$$
\begin{aligned}
\omega(\psi, \bar{D}, \delta) & \leqslant 2 N_{1}(B+|\log (\delta / 2)|)^{-\nu} \leqslant 2 N_{1}(B+|\log \delta|)^{-\nu} \\
& =2 N_{1}\left(\frac{1+|\log \delta|}{B+|\log \delta|}\right)^{\nu} \cdot(1+|\log \delta|)^{-\nu}
\end{aligned}
$$

Так как $(1+x) /(B+x) \leqslant \max \{1,1 / B\}$ при любом $x \in[0,+\infty)$, то второе неравенство из следствия 4.1 также получено.

Следствием теоремы 4.1 и лемм 2.1 и 2.4 является также и следующая теорема о граничной “гладкости" конформных отображений круга на ограниченные области с произвольными спрямляемыми жордановыми границами. Доказательство ее аналогично доказательству теоремы 4.2. Именно, вместо $d_{g}(x)$, использованного в доказательстве неравенства (4.13), теперь надо использовать $m_{g}(x)$ и т.п. В доказательстве же неравенства (4.20) теперь следует взять $m m_{c}(x)$, а вместо леммы 2.1 использовать лемму 2.4 .

Теорема 4.3. Пусть $w=\psi(z)$ - однолистное конформное отображение единичного круга $D$ на ограниченную область $G$ со спрямляемой жордановой границей $\Gamma \in J(g, \varepsilon) \cap J_{0}(h, \eta)$. Тогда

$$
\omega(\psi, \bar{D}, \delta) \leqslant B_{1} g\left(\sqrt{M_{h}^{-1}(\log \delta)}\right), \quad \delta \geqslant 0
$$

(в правой части этого неравенства можно вместо g написать $h$ ). Eсли при этом $h(x) \leqslant c x, x \in[0, \eta], c=$ const $>1$, то при всех $\delta \geqslant 0$ и $>>1$ верно и существенно более сильное неравенство

$$
\omega(\psi, \bar{D}, \delta) \leqslant B_{2} \delta^{\alpha(c)}, \quad \delta \geqslant 0, \quad \alpha(c):=\frac{1}{2 \pi S(c)} \equiv \frac{2}{\pi} \operatorname{arcctg}(4 B(c))
$$

(см. (2.21)). Из этих соотношений и неравенства $S(c)<\bar{S}(c), c>1, S(1)=$ $\bar{S}(1)=1 /(2 \pi)$, получаем при $\delta \in[0,1]$ неравенство (см. (2.19) u $(2.20))$

$$
\omega(\psi, \bar{D}, \delta) \leqslant B_{3} \delta^{\bar{\alpha}(c)}, \quad \bar{\alpha}(c):=\frac{1}{2 \pi \bar{S}(c)}=\frac{4}{(c+1)^{2} \bar{s}(c)},
$$

$\left(1-6.5 \cdot 10^{-3}\right) \alpha(c)<\bar{\alpha}(c)<\alpha(c), c>1$. Положительные величины $B_{1}, B_{2}, B_{3}$ не зависят от $\delta \geqslant 0$, причем при $\delta \in[0,1]$ можно считать, что $B_{3}=B_{2}$. 
Неравенство $\left(1-6.5 \cdot 10^{-3}\right) \alpha(c)<\bar{\alpha}(c)$ для всех $c>1$ вытекает из последнего утверждения леммы 2.4 .

Отметим, что $\alpha(c) \nearrow 1=\alpha(1)$ и $\bar{\alpha}(c) \nearrow 1=\bar{\alpha}(1)$ при $c \searrow 1$, в то время как в упомянутом выше неравенстве Варшавского из [3] было $\alpha=2 /(c+1)^{2}<1 / 2$ $(c>1)$.

СлЕДСтвиЕ 4.2. Если при условиях теоремы имеем $h(x) \leqslant c x^{k}$, где $c>1$, $0<k \leqslant 1$, то при $k=1$ верно неравенство $\omega(\psi, \bar{D}, \delta) \leqslant B_{2} \delta^{\alpha(c)}, \delta \geqslant 0$, a при $0<k<1$ u $0 \leqslant \delta \leqslant 2$ - неравенство $\omega(\psi, \bar{D}, \delta) \leqslant B_{4}(1+|\log \delta|)^{-\nu(k)}$ с $\nu(k)=k^{2} /(2-2 k)\left(B_{2}, B_{4}\right.$ положительны и не зависят от $\left.\delta\right)$.

ЗАмечАниЕ 4.5. Отметим, что в неравенствах (4.13) и (4.19) множители $g\left(\sqrt{D_{g}^{-1}(1 ; \log \delta)}\right)$ и $g\left(\sqrt{M_{h}^{-1}(1 ; \log \delta)}\right)$ зависят от $g, h$ и $\delta$, но не зависят от $\psi$, в то время как коэффициенты $B$ и $B_{1}$ не зависят от $\delta$, но зато зависят от $\psi$.

ЗАмЕчАНИЕ 4.6. Как и в Замечании 4.4, отметим, что при рассмотрении класса $J_{0}(h, \eta)$ с любой допустимой (см. (4.10)) функцией $h(x)$ имеем

$$
\begin{gathered}
h(x) \geqslant x, \quad y=m_{h}(x) \geqslant x, \quad x=m_{h}^{-1}(y) \leqslant y, \quad \frac{1}{m_{h}^{-1}(y)} \geqslant \frac{1}{y}, \\
-Y=-M_{h}(1 ; X) \geqslant \log \frac{1}{X}, \quad Y=M_{h}(1 ; X) \leqslant \log (X), \quad 0<x \leqslant 1, \\
x=M_{h}^{-1}(y) \geqslant e^{y} ;
\end{gathered}
$$

$g\left(\sqrt{M_{h}^{-1}(\log \delta)}\right) \geqslant \sqrt{\delta}, \delta \in[0,1]$. Таким образом, неравенство (4.6) не улучшилось от этой конкретизации $u(x)$, так что при всех допустимых $g(x)$ имеем неравенство

$$
g\left(\sqrt{M_{h}^{-1}(1 ; \log \delta)}\right) \geqslant \sqrt{\delta}, \quad \delta \in[0,1] .
$$

Это неравенство нам также понадобится в $\S 6$.

\section{§ 5. Отображения жордановых областей друг на друга}

Теорема 5.1. Пусть $w=f(z)$ - однолистное конформное отображение ограниченной односвязной области $G$ с жордановой гранищей $\partial G \in J(g, \varepsilon)$ на ограниченную односвязную область $Q$ с жордановой границей $\partial Q \in J(q, \eta)$, где $\varepsilon>0, \eta>0$. Тогда

$$
\omega(f, \bar{G}, \delta) \leqslant A^{*} q\left(\sqrt{D_{q}^{-1}\left(2^{-1} \log g(\delta)\right)}\right), \quad \delta \geqslant 0 .
$$

Если при этом $\partial Q \in J_{0}(h, \vartheta)$, то

$$
\omega(f, \bar{G}, \delta) \leqslant B^{*} q\left(\sqrt{M_{h}^{-1}\left(2^{-1} \log g(\delta)\right)}\right), \quad \delta \geqslant 0,
$$

и если при этом $h(x) \leqslant c x, x \in[0, \vartheta], c>1, \vartheta>0$, то также

$$
\omega(f, \bar{G}, \delta) \leqslant C^{*}(g(\delta))^{\alpha(c) / 2}, \quad \delta \geqslant 0 .
$$

Здесь положительные величины $A^{*}, B^{*}, C^{*}$ не зависят от $\delta, a \alpha(c)-\kappa а к ~$ в (4.20). 
СлЕДСТвИЕ 5.1. Если при условиях теоремы 5.1 верны неравенства $g(x) \leqslant$ $M x^{m}$ u $q(x) \leqslant N x^{n}$, где $M, N>0$ u $m, n \in(0,1]$ постоянны, то $\omega(f, \bar{G}, \delta) \leqslant$ $E \delta^{k} c k=m(\pi(N+1))^{-2}$ npu $0<m \leqslant 1, n=1 u \delta \geqslant 0 ; \omega(f, \bar{G}, \delta) \leqslant F(1+|\log \delta|)^{r}$ c $r=n^{2} /(2-2 n)$ nри $0<m \leqslant 1,0<n<1 u 0 \leqslant \delta \leqslant \operatorname{diam}(G)(\operatorname{diam}(G)-$ диаметр $G)$. Здесь положительные величины $E$ и $F$ не зависят от $\delta$.

СлеДСТвИЕ 5.2. Пусть гладкий контур $\Gamma=\partial G$ является ляпуновским или хотя бы обобщенно ляпуновским (см. замечание 3.1). Тогда в неравенствах (5.1) и (5.2) можно справа убрать множитель $2^{-1}$, а правая часть в (5.3) примет вид $C^{*} \delta^{\alpha(c)}$.

ДоКАЗАТЕЛЬСТво теоремы 5.1. Отображение $w=f(z)$ представим в виде суперпозиции какого-либо однолистного конформного отображения $\zeta=\varphi(z)$ области $G$ на единичный круг $D$ и однолистного конформного отображения $w=\psi(\zeta):=f\left(\varphi^{-1}(\zeta)\right)$ круга $D$ на область $Q$. Так как $f(z)=\psi(\varphi(z))$, то $\omega(\delta):=$ $\omega(f, \bar{G}, \delta) \leqslant \omega(\psi, \bar{D}, \omega(\varphi, \bar{G}, \delta))$. По теореме 3.1 (см. (3.1)) $\omega(\varphi, \bar{G}, \delta) \leqslant A_{1} \sqrt{g(\delta)}$, $\delta \geqslant 0$. Подставляя это неравенство в предыдущее и пользуясь свойством $(2.2)$ модуля непрерывности, получаем неравенство

$$
\omega(\delta) \leqslant \omega\left(\psi, \bar{D}, A_{1} \sqrt{g(\delta)}\right) \leqslant\left(A_{1}+1\right) \omega(\psi, \bar{D}, \sqrt{g(\delta)}) .
$$

Для доказательства (5.1) осталось воспользоваться неравенством (4.13) из теоремы 4.2, а для доказательства неравенств (5.2) и (5.3) - соответственно неравенствами (4.19) и (4.20) из теоремы 4.3. Теорема 5.1 доказана полностью.

Что касается следствий этой теоремы, то следствие 5.1 получается непосредственно из неравенств (5.1)-(5.3), а следствие 5.2 - из замечания 3.1 о липшицевости функций $\varphi(z)$ и $\psi(w)=\varphi^{-1}(w)$, возникающих при доказательстве теоремы 5.1 .

Заметим также, что аналогичным рассуждением могут быть уточнены и неравенства из следствия 5.1, если в условии этого следствия дополнительно потребовать, чтобы область $G$ имела обобщенно ляпуновскую границу (см. замечание 3.1 ).

\section{§ 6. Локальные формы теорем}

Приводимые здесь утверждения являются локальными формами теорем из $\S \S 3-5$.

ТеОрема 6.1. Пусть $G$-односвязная область плоскости $\mathbb{C}, \Gamma-$ ограниченная открытая достижимая жорданова дуга ее границы $\partial G, \Gamma \in J(g, \varepsilon), \varepsilon>0$. Тогда любое однолистное конформное отображение $w=\varphi(z)$ области $G$ на единичный круг $D$ продолжается на замыкание дуги $Г$ до гомеоморфизма $\bar{\Gamma}$ замыканию $\bar{\gamma}$ дуги $\gamma=\varphi(\Gamma)$ и гомеоморфизма множества $G \cup \Gamma$ множеству $D \cup \gamma$, причем

$$
\omega(\varphi, \bar{\Gamma}, \delta) \leqslant A_{1} \sqrt{g(\delta)}, \quad \delta \geqslant 0 .
$$

При этом для каждого компакта $E \subset G \cup \Gamma$ справедливо неравенство

$$
\omega(\varphi, E, \delta) \leqslant A_{1}(E) \sqrt{g(\delta)}, \quad \delta \geqslant 0 .
$$

Здесь конечные положительные величины $A_{1} u A_{1}(E)$ не зависят от $\delta$. 
ЗАмЕчАНИЕ 6.1. Пусть выполнены условия теоремы 6.1. Если $R$ - обычное (евклидово) расстояние точки $b=\varphi^{-1}(0)$ до дуги $\Gamma$, а число $r \in(0, \varepsilon]$ удовлетворяет неравенству $9 g(r)<2 \pi R$, то

$$
\omega(\varphi, \bar{\Gamma}, \delta) \leqslant A_{0}(\varphi) \sqrt{g(\delta)}, \quad \delta \in[0, r], \quad A_{0}(\varphi)=3 K_{\Lambda} \sqrt{\left|\varphi^{\prime}(b)\right|}
$$

$K_{\Lambda}-$ постоянная Лаврентьева из п. 2.3.

ДОКАЗАТЕЛЬСТВо ТЕОРЕМЫ 6.1. О продолжимости отображения $w=\varphi(z)$ с $G$ на замкнутую достижимую жорданову дугу $\bar{\Gamma} \subset \partial G$ до гомеоморфизма множества $G \cup \Gamma$ множеству $D \cup \gamma$ и гомеоморфизма замкнутой дуги $\bar{\Gamma}$ замкнутой дуге $\bar{\gamma}$ говорит локальная форма теоремы Каратеодори (см. п. 2.2).

Справедливость неравенств (6.1) и (6.3) непосредственно следует из леммы 2.9. Докажем неравенство (6.2).

А) Пусть сначала компакт $E$ целиком лежит в $G$. Окружим его гладким жордановым контуром $L \subset G \backslash E$. Тогда при $z, t \in E$ имеем равенство

$$
\varphi(z)-\varphi(t)=\frac{1}{2 \pi i} \int_{L} \varphi(\zeta)\left(\frac{1}{\zeta-z}-\frac{1}{\zeta-t}\right) d \zeta=\frac{z-t}{2 \pi i} \int_{L} \frac{\varphi(\zeta) d \zeta}{(\zeta-z)(\zeta-t)}
$$

так что $|\varphi(z)-\varphi(t)| \leqslant A|z-t|$ при $A=M(\varphi, L)|L| /\left(2 \pi \rho^{2}\right)$, где $M(\varphi, L)=$ $\max \{|\varphi(\zeta)|: \zeta \in L\}, \rho-$ расстояние между $E$ и $L$. Поэтому $\omega(\varphi, E, \delta) \leqslant A \delta$. Отсюда $\omega(\varphi, E, \delta) \leqslant A \sqrt{\delta}$ при $0 \leqslant \delta \leqslant 1$ и $\omega(\varphi, E, \delta) \leqslant(A \sqrt{\operatorname{diam}(E)}) \sqrt{\delta}$ при всех $\delta \geqslant 0$. Остается вспомнить, что $\delta \leqslant g(\delta)$ (см. п. 1.2 ), так что $\sqrt{\delta} \leqslant \sqrt{g(\delta)}$. Случай $E \subset G$ исчерпан.

В) Теперь пусть $E \cap \Gamma \neq \varnothing$, и $\bar{\Sigma}$ - минимальная замкнутая поддуга дуги $\Gamma$ (возможно, вырожденная в единственную точку), содержащая компакт $E \cap \Gamma$. До конца доказательства этой теоремы будем пользоваться обозначениями и утверждениями леммы 2.10 .

По лемме 2.10 имеется такая односвязная ограниченная область $G\left(t^{\prime}, t^{\prime \prime}\right) \subset G$ с жордановой границей $\Gamma\left(t^{\prime}, t^{\prime \prime}\right)$, что $E \subset G\left(t^{\prime}, t^{\prime \prime}\right) \cup \Gamma$ и $\Gamma\left(t^{\prime}, t^{\prime \prime}\right)$ обладает следующими свойствами:

1) $\Gamma\left(t^{\prime}, t^{\prime \prime}\right) \cap \partial G=\smile^{\prime} t^{\prime \prime}$, где $\smile t^{\prime} t^{\prime \prime}$ - открытая достижимая дуга на $\Gamma$ с концами $t^{\prime}, t^{\prime \prime} \in \Gamma \backslash \bar{\Sigma}$, причем $\bar{\Sigma} \subset \smile t^{\prime} t^{\prime \prime}$ и $\smile t^{\prime} t^{\prime \prime} \subset \Gamma$;

2) $\Gamma\left(t^{\prime}, t^{\prime \prime}\right) \cap G:=\Lambda\left(t^{\prime}, t^{\prime \prime}\right)-$ открытая дуга на кривой $\Gamma\left(t^{\prime}, t^{\prime \prime}\right)=\smile t^{\prime} t^{\prime \prime} \cup$ $\left\{t^{\prime}, t^{\prime \prime}\right\} \cup \Lambda\left(t^{\prime}, t^{\prime \prime}\right)$, причем ее замыкание $\bar{\Lambda}\left(t^{\prime}, t^{\prime \prime}\right)$ - дважды гладкая жорданова дуга с теми же концами $t^{\prime}, t^{\prime \prime}$ - находится на положительном расстоянии от $E$ и от $\partial G \backslash \Gamma$.

Важно, что $\Gamma\left(t^{\prime}, t^{\prime \prime}\right) \in J\left(g^{*}, \eta^{*}\right)$ с $g^{*}(x)=g(x)+x \leqslant 2 g(x)$ и некоторым числом $\eta^{*} \in(0, \eta]$ леммы 2.10 .

Положим

$$
\begin{aligned}
\tau^{\prime}:=\varphi\left(t^{\prime}\right), \quad \tau^{\prime \prime}:=\varphi\left(t^{\prime \prime}\right), \quad G_{1}:=\varphi\left(G\left(t^{\prime}, t^{\prime \prime}\right)\right), \quad \Gamma_{1}:=\varphi\left(\Gamma\left(t^{\prime}, t^{\prime \prime}\right)\right), \quad E_{1}:=\varphi(E), \\
\gamma:=\varphi(\Gamma), \quad \sigma:=\varphi(\Sigma), \quad \bar{\sigma} \subset \smile \tau^{\prime} \tau^{\prime \prime}, \quad \smile_{\tau^{\prime} \tau^{\prime \prime}} \subset \gamma \subset C .
\end{aligned}
$$

Пусть $\zeta=f(w)$ - какое-либо однолистное конформное отображение области $G_{1}$ на круг $D$, оставляющее неподвижными концы дуги $\tau^{\prime} \tau^{\prime \prime}$. В силу принципа симметрии функция $f(w)$ аналитически и однолистно продолжается через дугу $\smile \tau^{\prime} \tau^{\prime \prime}$ в некоторую односвязную жорданову область $D^{+}$, лежащую 
вне $\bar{D}$, в состав границы которой входит эта дуга. Обозначим через $L$ некоторую дважды гладкую замкнутую жорданову кривую со следующими свойствами: а) в состав $L$ входит дуга $\bar{\sigma}$; b) если $U$ - внутренность контура $L$, то $E_{1} \subset \bar{U} \subset G_{1} \cup \bar{\sigma}$.

Рассмотрим функцию $\zeta=\Phi(z):=f(\varphi(z))$, отображающую конформно и однолистно область $G\left(t^{\prime}, t^{\prime \prime}\right) \subset G$ с границей $\Gamma\left(t^{\prime}, t^{\prime \prime}\right) \in J\left(g^{*}, \eta^{*}\right)$ на круг $D$ $\left(g^{*}(x) \leqslant 2 g(x) ;\right.$ см. лемму 2.10). По теореме 3.1 имеем неравенство

$$
\omega\left(\Phi, \overline{G\left(t^{\prime}, t^{\prime \prime}\right)}, \delta\right) \leqslant A_{1} \sqrt{2 g(\delta)}, \quad \delta \geqslant 0 .
$$

Так как $f(w)$ аналитична и однолистна в окрестности замкнутой области $\bar{U}$ с дважды гладкой границей $L$, то граница $f(L)$ области $f(U) \subset D$ также дважды гладкая, а $f^{-1}(\zeta)$ аналитична и однолистна в некоторой окрестности замкнутой области $\overline{f(U)}$. Поэтому $\mid\left(f^{-1}(\underline{\zeta)})^{\prime} \mid \leqslant M\right.$ при некотором конечном $M>0$ и любом $\zeta \in \overline{f(U)}$, откуда $\omega\left(f^{-1}, \overline{f(U)}, \delta\right) \leqslant M_{1} \delta, \delta \geqslant 0$ ( $M_{1}$ не зависит от $\delta$ ). Так как $\varphi(z) \equiv f^{-1}(\Phi(z)), z \in E$, то отсюда и из (6.4) следует, что $\omega(\varphi, E, \delta) \leqslant M_{1} A_{1} \sqrt{2 g(\delta)}$ при $\delta \geqslant 0$.

Теорема 6.1 доказана полностью.

ТеОрема 6.2. Пусть $w=\psi(z)$ - однолистное конформное отображение единичного круга $D$ на односвязную область $G \subset \mathbb{C}, \Gamma$ - ограниченная открытая достижимая жорданова дуга границь этой области, $Г \in J(g, \varepsilon)$, $\gamma=\psi^{-1}(\Gamma)$. Тогда для каждого компакта $E \subset D \cup \gamma$ верно неравенство

$$
\omega(\psi, E, \delta) \leqslant B g\left(\sqrt{D_{g}^{-1}(\log \delta)}\right), \quad \delta \geqslant 0 .
$$

Если при этом $\Gamma \in J_{0}(h, \eta)$ при некотором $\eta>0$, то такюе

$$
\omega(\psi, E, \delta) \leqslant B_{0} g\left(\sqrt{M_{h}^{-1}(\log \delta)}\right), \quad \delta \geqslant 0
$$

(в правой части этого неравенства вместо $g$ можно взять $h$ ). Если $к$ тому же $h(x) \leqslant c x, x \in[0, \eta], c=\mathrm{const} \geqslant 1$, то верно и существенно более сильное неравенство

$$
\omega(\psi, E, \delta) \leqslant B_{1} \delta^{\alpha(c)}, \quad \delta \geqslant 0 .
$$

Здесъ положительные величинь $B, B_{0}, B_{1}$ не зависят от $\delta, a \alpha(c)$ - как в (4.20).

ЗАмечАниЕ 6.2. Неравенства (6.5)-(6.7), вообще говоря, не верны для компакта-дуги $E=\bar{\gamma}$ (сравните с неравенством (6.1)!). Нужный пример предоставляют при малых $s>0$ области $G_{s}$ следующего вида: $G_{s}:=\left\{w=\psi_{s}(z):|z|<1\right\}$, где $\psi_{s}(z):=|z+1|^{s} e^{i s \arg (z+1)},-\pi / 2<\arg (z+1)<\pi / 2,-$ однозначная и однолистная аналитическая ветвь функции $w=(z+1)^{s}$ в единичном круге $D$ (доказательство этого, включая выбор дуг $\Gamma=\Gamma_{s}$ и $\gamma=\gamma_{s}:=\psi_{s}^{-1}\left(\Gamma_{s}\right)$, см. после доказательства теоремы 6.2).

ЗАмЕчАНИЕ 6.3. Потребовав от достижимой жордановой дуги Г лишь ее спрямляемости (см. теорему 6.2), можно утверждать, что граничная функция $\psi(\zeta), \zeta \in \gamma$, дифференцируема вдоль $\gamma$ почти в каждой точке этой дуги как функция с конечным полным изменением $\operatorname{Var}_{\gamma}(\psi)=|\Gamma|$. На самом деле 
при этом $f(z)$ дифференцируема еще и по $z \in D \cup \gamma$ также почти в каждой точке $\zeta_{0} \in \gamma$. Более общее утверждение: если $\psi(\zeta), \zeta \in \gamma$, имеет в каждой точке $\zeta_{0}$ множества $E_{n} \subset \gamma$ дифференциал Пеано $n$-го порядка, $n \geqslant 1$, по переменной $\zeta \in \gamma$ (это означает наличие соотношения $\psi(\zeta)-\psi\left(\zeta_{0}\right)=P\left(\zeta-\zeta_{0}\right)+o\left(\left(\zeta-\zeta_{0}\right)^{n}\right)$ при $\left.\zeta-\zeta_{0} \rightarrow 0\right)$, то почти в каждой точке $\zeta_{0} \in E_{n}$ функция $\psi(z)$ имеет дифференциал Пеано того же порядка $n$ также и по переменной $z \in D \cup \gamma$ (см. [17]).

ДоказАтельСтво теоремы 6.2. Так как случай $\Gamma=\partial G$ исчерпывается теоремами 4.2 и 4.3, то будем считать, что $\Gamma \neq \partial G$.

J1) Пусть сначала $\operatorname{diam}(\Gamma) \leqslant \varepsilon$ и $\Gamma \in J(g, \varepsilon)$. Тогда $\Gamma \in J(g)$. Если рассматривается случай $\Gamma \in J_{0}(h, \eta)$, то если мы доказываем неравенство (6.6), дополнительно к условию $\operatorname{diam}(\Gamma) \leqslant \varepsilon$ требуем выполнение и условия $|\Gamma| \leqslant \eta$; тогда $\Gamma \in J(g) \cap J_{0}(h)$. Пусть, кроме того, открытая дуга $\sigma \subset C$ такова, что $\bar{\sigma} \subset \gamma$ и $|\sigma| \leqslant \pi$. Если Sec - замкнутый сектор круга $D$, опирающийся на дугу $\bar{\sigma}$, то в силу сказанного величина угла при его вершине не превосходит $\pi$ и $\mathrm{Sec}-$ выпуклый компакт. Пусть $\rho$ - евклидово расстояние между $\bar{\sigma}$ и $C \backslash \gamma, a=1$ (см. (1.6) и лемму 2.6), $\delta \leqslant r:=\rho /(2 e)$. Для сокращения записи положим

$$
\psi(z, t):=\psi(z)-\psi(t)
$$

При указанных условиях и обозначениях докажем неравенства (6.5) и (6.6) для случая $E=\mathrm{Sec}$.

Пусть $\sigma^{*}$ - такая открытая дуга на $\gamma$, что $\bar{\sigma} \subset \sigma^{*} \subset \overline{\sigma^{*}} \subset \gamma$. Тогда из теоремы 4.1 и лемм 2.1, 2.4 получим (как при доказательстве теоремы 4.2) неравенство (4.5), в котором точки $t \in \overline{\sigma^{*}}$ и $z \in \bar{D}$ - любые при условии $|z-t| \leqslant$ $2 r=\rho / e$, а вместо $U^{-1}(a ; y)$ стоит (напомним, что у нас $\left.a=1\right) D_{g}^{-1}(1 ; y)$, либо $M_{h}^{-1}(1 ; y)$, либо $M M_{c}^{-1}(1 ; y)$ - в зависимости от того, какое из следующих трех условий выполнено соответственно: 1) $\Gamma \in J(g)$; 2$) \Gamma \in J_{0}(h)$; 3$) \Gamma \in J_{0}(h)$, где $h(x) \leqslant c x, x \geqslant 0$ (т.е. $\left.\Gamma \in J_{0}(c \cdot)\right)$. В результате этих замен мы из неравенства

$$
|\psi(z, t)| \leqslant 2 g\left(\sqrt{U^{-1}\left(1 ; \log \left(E_{\sigma^{*}}(\rho) e|z-t|\right)\right)}\right),
$$

где $t \in \overline{\sigma^{*}}, z \in \bar{D},|z-t| \leqslant \rho / e, E_{\sigma^{*}}(\rho)=E\left(\psi, \gamma, \sigma^{*}, u, 1 ; \rho\right)$ (см. (4.2)), получаем три неравенства при указанных $t, z$ и функциях $u(x)=d_{g}(x)$ (если $\Gamma \in J(g)$ ), или $u(x)=m_{h}(x)$ (если $\left.\Gamma \in J_{0}(h)\right)$, или $u(x)=m_{h}(x)$ при $h(x)=c x$ (в этом случае $\left.u(x)=m m_{c}(x)=\pi S(c) x\right)$ - см. (4.8), (4.10) и (4.11).

Сейчас мы перенесем эти три неравенства на некоторый выпуклый замкнутый сектор Sec**, покрывающий наш сектор Sec и опирающийся на такую замкнутую дугу $\overline{\sigma^{* *}} \subset \gamma$, что, во-первых, $\bar{\sigma} \subset \sigma^{* *}$ и $\overline{\sigma^{* *}} \subset \sigma^{*}$ и, во-вторых, на граничных радиусах $R^{\prime}, R^{\prime \prime}$ сектора Sec** (образующих угол, величина которого меньше $\pi$ ) выполняется условие (2.29) леммы 2.8 и если при этом $\Gamma \in J_{0}(h)$, то выполняются еще и условия (2.31) этой леммы с $R(t)=R^{\prime}$ и $R(t)=R^{\prime \prime}$.

Для построения такого сектора $\mathrm{Sec}^{* *}$ воспользуемся леммой 2.8. Пусть $\sigma^{\prime}$ и $\sigma^{\prime \prime}$ - две открытые дуги, на которые распадается открытое (на $C$ ) множество $\sigma^{*} \backslash \bar{\sigma}$. Эти дуги лежат на $\overline{\sigma^{*}}$ по разные стороны от $\bar{\sigma}$, замыкания их образов $\overline{\Sigma^{\prime}}=\psi\left(\overline{\sigma^{\prime}}\right)$ и $\overline{\Sigma^{\prime \prime}}=\psi\left(\overline{\sigma^{\prime \prime}}\right)$ лежат на $Г$ и принадлежат классу $J(g)$ и, возможно, еще и классу $J_{0}(h)$ (если $\left.\Gamma \in J_{0}(h)\right)$. По лемме 2.8 найдутся радиусы $R^{\prime}$ и $R^{\prime \prime}$ круга $D$, оканчивающиеся на $\sigma^{\prime}$ и $\sigma^{\prime \prime}$ соответственно, на каждом из которых для разностей $\psi(z, t)=\psi(z)-\psi(t)$ при $z, t \in R^{\prime}$ и при $z, t \in R^{\prime \prime}$ 
выполнены неравенства

$$
|\psi(z, t)| \leqslant A_{1} \sqrt{|z-t|}, \quad \Gamma \in J(g), \quad|\psi(z, t)| \leqslant A_{2}|z-t|, \quad \Gamma \in J(g) \cap J_{0}(h),
$$

где положительные величины $A_{1}$ и $A_{2}$ не зависят от $z, t$.

Обозначим через Sec** замкнутый сектор, ограниченный радиусами $R^{\prime}, R^{\prime \prime}$ и замкнутой дугой $\overline{\sigma^{* *}} \subset \sigma^{*}$, соединяющей концы этих радиусов.

Оценим $\psi(z, t)$ при всех возможных положениях точек $z, t \in \partial \operatorname{Sec}^{* *}$ (с точностью до замены $z$ на $t$ и $t$ на $z$ ) при условии $|z-t| \leqslant \delta \leqslant r=2^{-1} \rho / e$.

a) При $t \in \overline{\sigma^{* *}}, z \in \overline{\sigma^{* *}} \cup R^{\prime} \cup R^{\prime \prime}$ нужную оценку дает неравенство (6.8).

b) При $z, t \in R^{\prime}$ или $z, t \in R^{\prime \prime}$ верны неравенства (6.9).

c) Если $z \in R^{\prime}, t \in R^{\prime \prime}$ и $z, t \in D_{r}$, где

$$
D_{r}=\overline{D(0,1-r)}:=\{\zeta:|\zeta| \leqslant 1-r\},
$$

то $|\psi(z, t)| \leqslant M|z-t| \leqslant M \delta$, где $M=\max \left\{\left|\psi^{\prime}(\zeta)\right|:|\zeta| \leqslant 1-r\right\}$.

d) Пусть $z \in R^{\prime}, t \in R^{\prime \prime}$ и $1-r<|t|<1$, а $z^{\prime}$ - точка пересечения $R^{\prime}$ с окружностью $C_{r}, t^{\prime}$ - основание перпендикуляра, опущенного из $z^{\prime}$ на диаметр круга $D$, включающий радиус $R^{\prime \prime}$ (напомним, что угол между $R^{\prime}$ и $R^{\prime \prime}$ меньше $\pi$ ). Очевидно, что $\left[z^{\prime}, t^{\prime}\right] \subset D_{r}$ и что $d:=\left|z^{\prime}-t^{\prime}\right|<|z-t| \leqslant \delta \leqslant r$, так что $|z-t|>d$. Положим $N^{\prime}=\left[\left|z-z^{\prime}\right| / d\right]+1$ и $N^{\prime \prime}=\left[\left|t-t^{\prime}\right| / d\right]+1$, где $[x]$ обозначает целую часть числа $x \geqslant 0$. Очевидно, $N^{\prime} \leqslant(1 / d)+1, N^{\prime \prime} \leqslant(2 / d)+1$. Отметим на отрезке $\left[z, z^{\prime}\right]$ точки $z_{0}=z, z_{1}, \ldots, z_{N^{\prime}}=z^{\prime}$ (занумеровав их в порядке возрастания их расстояний от $z)$, а на отрезке $\left[t^{\prime}, t\right]$ отметим точки $t_{0}=t^{\prime}$, $t_{1}, \ldots, t_{N^{\prime \prime}}=t$ (занумеровав их в порядке возрастания их расстояний от $\left.t^{\prime}\right)$. При этом позаботимся о том, чтобы расстояния между соседними точками в каждой из этих последовательностей не превосходили $d$. Тогда (напомним, что в рассматриваемом случае $d<|z-t| \leqslant \delta \leqslant r<1$ ) получим, что

$$
\begin{gathered}
|\psi(z, t)| \leqslant \sum_{m=1}^{N^{\prime}}\left|\psi\left(z_{m}, z_{m-1}\right)\right|+\left|\psi\left(t^{\prime}, z^{\prime}\right)\right|+\sum_{n=1}^{N^{\prime \prime}}\left|\psi\left(t_{n}, t_{n-1}\right)\right|, \\
|\psi(z, t)| \leqslant N^{\prime} A_{1} \sqrt{d}+M d+N^{\prime \prime} A_{1} \sqrt{d} \leqslant A \sqrt{\delta}, \quad \Gamma \in J(g), \\
|\psi(z, t)| \leqslant N^{\prime} A_{2} d+M d+N^{\prime \prime} A_{2} d \leqslant B \delta, \quad \Gamma \in J_{0}(h),
\end{gathered}
$$

где величины $A$ и $B$ не зависят от $\delta \leqslant r$.

Итак, рассмотрены все возможные случаи взаимного расположения точек $z$ и $t$ на границе сектора $\operatorname{Sec}^{* *}$ (с точностью до замены пары $z, t$ парой $t, z$ ).

Из рассуждений пп. а)-d) следует, что для любых $z, t \in \partial \mathrm{Sec}^{* *}$ при условии $|z-t| \leqslant \delta \leqslant r$ (напомним, что $r<1$ ) справедливо неравенство

$$
|\psi(z, t)| \leqslant F_{1} \sqrt{\delta}+2 g\left(\sqrt{U^{-1}\left(\log \left(b_{u}|z-t|\right)\right)}\right),
$$

где $F_{1}>0$ не зависит от $\delta$. При этом если $\Gamma \in J(g)$, то $u(x)=d_{g}(x)$ (см. (4.8)), $U^{-1}(1 ; y)=D_{g}^{-1}(1 ; y)$ и $b_{u}=E\left(\psi, \gamma, \sigma^{*}, d_{g}, 1 ; \rho\right) e$ (определение $E(\cdot)$ см. (4.2)); если же еще и $\Gamma \in J_{0}(h)$, то $u=m_{h}$ (см. (4.10)), $U^{-1}(1 ; y)=M_{h}^{-1}(1 ; y)$ и $b_{u}=E\left(\psi, \gamma, \sigma^{*}, m_{h}, 1 ; \rho\right) e$, и если при этом $h(x) \leqslant c x, c=$ const $\geqslant 1$, то $u(x)=$ $m m_{c}(x)=\pi S(c) x$ (см. (4.11)), $U^{-1}(1 ; y)=M M_{c}^{-1}(1 ; y)=e^{y /(\pi S(c))}$ (см. (4.12)). 
Далее, если число $\delta \in[0, r]$ удовлетворяет условию $b_{u} \delta \in[0, r]$, то в силу замечания 4.3 к теореме 4.1 (см. (4.6)) имеем оценку

$$
\sqrt{b_{u} \delta} \leqslant g\left(\sqrt{U^{-1}\left(1 ; \log \left(b_{u} \delta\right)\right)}\right)
$$

а отсюда и из (6.10) получаем неравенство

$$
\omega\left(\psi, \partial \operatorname{Sec}^{* *}, \delta\right) \leqslant F_{2} g\left(\sqrt{U^{-1}\left(\log \left(b_{u} \delta\right)\right)}\right), \quad \delta \in\left[0, \frac{r}{b_{u}^{\prime}}\right],
$$

где $b_{u}^{\prime}:=\max \left\{b_{u}, 1\right\}$ и $F_{2}$ не зависят от $\delta$.

В силу выпуклости $\operatorname{Sec}^{* *}$ к $\omega\left(\psi, \operatorname{Sec}^{* *}, \delta\right)$ применимо неравенство Тамразова (2.4). Так как Sec $\subset$ Sec ${ }^{* *}$, то неравенства (6.5)-(6.7) доказаны при $\delta \leqslant r / b_{u}^{\prime}$ в случае произвольного замкнутого выпуклого сектора $E=\mathrm{Sec}$, опирающегося на какую-либо замкнутую поддугу $\bar{\sigma}$ дуги $\gamma$ при условии $\operatorname{diam}(\Gamma) \leqslant \varepsilon($ если $\Gamma \in J(g, \varepsilon))$, или, соответственно, при условиях $\left.\Gamma \in J(g, \varepsilon) \cap J_{0}(h, \eta)\right),|\Gamma| \leqslant \eta$.

Неравенство $\omega(\psi, \operatorname{Sec}, \delta) \leqslant B g\left(\sqrt{U^{-1}(\log \delta)}\right)$ на случай произвольного $\delta \geqslant 0$ обобщается (с использованием свойства (2.2) модуля непрерывности функции $\psi$ на выпуклом множестве Sec) аналогично тому, как это было сделано при доказательстве теоремы 4.2.

J2) В этом и следующем пунктах мы сведем случай произвольной открытой достижимой жордановой дуги $\Gamma \subset \partial G$, принадлежащей классу $J(g, \varepsilon)$ или принадлежащей одновременно классам $J(g, \varepsilon)$ и $J_{0}(h, \eta)$, к уже рассмотренным в п. J1) случаям $\Gamma \in J(g)$ и $\Gamma \in J(g) \cap J_{0}(h)$ соответственно.

Для этого сначала дугу $\Gamma \in J(g, \varepsilon)$ покроем конечным числом таких открытых дуг $\Gamma_{n} \subset \Gamma$, что $\Gamma_{n} \in J(g), n=1, \ldots, N=N(\Gamma, \varepsilon)$ (последнее обеспечивается условием $\left.\operatorname{diam}\left(\Gamma_{n}\right) \leqslant \varepsilon, n=1, \ldots, N=N(\Gamma, \varepsilon)\right)$. Если же $\Gamma \in J(g, \varepsilon) \cap J_{0}(h, \eta)$, то позаботимся о том, чтобы при каждом $n=1, \ldots, N=N(\Gamma, \varepsilon, \eta)$ выполнялось условие $\Gamma_{n} \in J(g) \cap J_{0}(h)$ (оно обеспечивается условием $\left|\Gamma_{n}\right| \leqslant \min \{\varepsilon, \eta\}$ на длины всех дуг $\left.\Gamma_{n}\right)$. Позаботимся еще о том, чтобы дуги $\Gamma_{n}$ с возрастанием $n$ двигались по Г в положительном направлении движения вдоль $Г$ и чтобы все дуги $\gamma_{n}:=\psi^{-1}\left(\Gamma_{n}\right)$ имели длины $\left|\gamma_{n}\right|<\pi$. При этом открытый сектор $\operatorname{Sec}(\gamma)$ круга $D$, опирающийся на дугу $\gamma=\psi^{-1}(\Gamma)$, окажется покрытым открытыми секторами $\operatorname{Sec}\left(\gamma_{n}\right)$, опирающимися на дуги $\gamma_{n}$ соответственно. Любые соседние секторы $\operatorname{Sec}\left(\gamma_{n-1}\right)$ и $\operatorname{Sec}\left(\gamma_{n}\right)$ пересекаются с “перехлестом", т.е. перекрываются, и их общая часть является также открытым сектором.

Возьмем какую-либо замкнутую дугу $\bar{\sigma} \subset \gamma$ и представим ее в виде объединения конечного числа замкнутых дуг $\bar{\sigma}_{n} \subset \gamma_{n}$, из которых каждые две соседние $\left(\bar{\sigma}_{n-1}\right.$ и $\left.\bar{\sigma}_{n}\right)$ имеют единственную общую (концевую) точку, которую обозначим через $t_{n}$ соответственно. По доказанному в п. J1) при каждом $n$ на замкнутом секторе $\operatorname{Sec}_{n}$, опирающемся на дугу $\bar{\sigma}_{n}$, модуль непрерывности функции $\psi$ при $\Gamma_{n} \in J(g)$ удовлетворяет неравенству

$$
\omega\left(\psi, \operatorname{Sec}_{n}, \delta\right) \leqslant B_{n} g\left(\sqrt{D_{g}^{-1}(\log \delta)}\right), \quad \delta \geqslant 0
$$

а если при этом еще и $\Gamma_{n} \in J_{0}(h)$, то этому неравенству и неравенству, получающемуся из него заменой $B_{n}$ и $D_{g}^{-1}$ на $B_{0, n}$ и $M_{h}^{-1}$ соответственно.

Теперь оценим $\omega(\psi, \operatorname{Sec}, \delta)$ для $\operatorname{Sec}:=\overline{\operatorname{Sec}(\sigma)}$ при $\bar{\sigma} \subset \gamma$ и $\Gamma=\psi(\gamma) \in J(g, \varepsilon)$ или же $\Gamma \in J(g, \varepsilon) \cap J_{0}(h, \eta)$. Зафиксируем некоторое число $\delta \in[0,2]$, возьмем 
какие-то две точки $z, t \in \mathrm{Sec}$ и соединим их отрезком $[z, t]$. Очевидно, если $[z, t] \subset \operatorname{Sec}($ что в случае выпуклости Sec верно для любой пары точек $z, t \in \mathrm{Sec}$ ), то

$$
|\psi(z, t)|=|\psi(z)-\psi(t)| \leqslant \sum_{[z, t]}\left|\psi\left(z_{n}\right)-\psi\left(z_{n-1}\right)\right|,
$$

где $\left\{z_{n}\right\}$ - множество всех точек пересечения радиусов $\left[0, t_{n}\right]$ с отрезком $[z, t]$, занумерованных в порядке их удаления от точки $z$, а $\sum_{[z, t]}$ означает суммирование по всем таким $n$, что $\left[z_{n-1}, z_{n}\right] \subset[z, t]$. Отсюда

$$
\begin{gathered}
\omega(\psi, \operatorname{Sec}, \delta) \leqslant \sum_{[z, t]} B_{n}^{\prime} g\left(\sqrt{U^{-1}(\log \delta)}\right)=B_{z, t}^{\prime} g\left(\sqrt{U^{-1}(\log \delta)}\right), \\
B_{z, t}^{\prime}:=\sum_{[z, t]} B_{n}^{\prime} \leqslant B^{\prime},
\end{gathered}
$$

где, как и выше, $U^{-1}=D_{g}^{-1}$ и $B_{n}^{\prime}=B_{n}$ при $\Gamma \in J(g, \varepsilon)$ либо $U^{-1}=M_{h}^{-1}$ и $B_{n}^{\prime}=B_{0, n}$ при $\Gamma \in J(g, \varepsilon) \cap J_{0}(h, \eta)$ соответственно, а $B^{\prime}$ зависит от $\psi, \Gamma$, ее покрытия $\left\{\Gamma_{n}\right\}$, выбора точек $t_{n}$ и дуги $\sigma$, но не от выбора числа $\delta \geqslant 0$ и точек $z, t \in \mathrm{Sec}$. В частности, этим доказано, что в случае выпуклости замкнутого сектора Sec при условии $\operatorname{Sec} \cap \partial D \subset \gamma$ верны неравенства (6.5)-(6.7) для $E=$ Sec.

J3) Теперь рассмотрим случай невыпуклого сектора Sec. Пусть для некоторой пары точек $z, t \in \mathrm{Sec},|z-t| \leqslant \delta$, имеем $[z, t] \not \subset$ Sec. Тогда множество $D \backslash$ Sec представляет собой открытый сектор некоторого раствора $\alpha=\alpha(\mathrm{Sec})<\pi$, граничные радиусы которого обозначим через $R^{\prime}$ и $R^{\prime \prime}$. Очевидно, при нашем выборе точек $z, t$ выполнено неравенство $\nu:=|\angle z 0 t|>\pi$.

Обозначим через $R$ радиус круга $D$, являющийся биссектрисой сектора Sec, a через $\mathrm{Sec}^{\prime}$ и $\mathrm{Sec}^{\prime \prime}$ - замкнутые подсекторы сектора Sec, ограниченные парами радиусов $R^{\prime}, R$ и $R, R^{\prime \prime}$ соответственно. Это выпуклые секторы, содержащие отрезки $[z, 0]$ и $[0, t]$ соответственно. Оценим длины этих отрезков.

Пусть отрезок $[z, t]$ пересекает радиусы $R^{\prime}$ и $R^{\prime \prime}$ в точках $z^{\prime}$ и $t^{\prime}$ соответственно; очевидно, $z^{\prime} \neq 0, t^{\prime} \neq 0$, так как иначе оказалось бы, что $[z, t] \subset \mathrm{Sec}$. Можно считать, что $0 \leqslant\left|z-z^{\prime}\right|<\left|z-t^{\prime}\right| \leqslant|z-t|$.

Если $\pi / 2 \leqslant \nu<\pi$, то в треугольнике $z 0 t$ угол $z 0 t$ строго больше двух других его углов, откуда $|z-0|,|0-t|<|z-t|$. Если же $0<\nu<\pi / 2$, то $\alpha \leqslant \nu<\pi / 2$. Опишем вокруг треугольника $z 0 t$ окружность. По теореме синусов ее диаметр равен $|z-t| / \sin \nu \leqslant|z-t| / \sin \alpha$. Итак, в любом случае имеем неравенство

$$
|z-0| \leqslant|z-t| k, \quad|0-t| \leqslant|z-t| k,
$$

где $k=k(\alpha):=1 / \sin \alpha$ при $0<\alpha \leqslant \pi / 2, k(\alpha)=1$ при $\pi / 2 \leqslant \alpha<\pi$. Учитывая неравенство $|\psi(z, t)| \leqslant|\psi(z, 0)|+|\psi(0, t)|$, выпуклость $\operatorname{Sec}^{\prime} \supset[z, 0]$ и $\operatorname{Sec}^{\prime \prime} \supset[0, t]$, из (2.2) получаем неравенства

$$
|\psi(z, t)| \leqslant \omega\left(\psi, \operatorname{Sec}^{\prime}, k \delta\right)+\omega\left(\psi, \operatorname{Sec}^{\prime \prime}, k \delta\right) \leqslant(k+1)\left(\omega\left(\psi, \operatorname{Sec}^{\prime}, \delta\right)+\omega\left(\psi, \operatorname{Sec}^{\prime \prime}, \delta\right)\right) .
$$

Так как секторы $\operatorname{Sec}^{\prime}$ и $\mathrm{Sec}^{\prime \prime}$ выпуклые и включаются в Sec, то отсюда и из утверждения в конце п. Ј2) получаем для $\omega(\psi, E, \delta)$ при $E=\operatorname{Sec}^{\prime}$ и $E=\operatorname{Sec}^{\prime \prime}$ 
неравенства (6.5) и (6.6) с дополнительным множителем $(k+1)$ справа (для любого $\delta \geqslant 0)$, а при $E=\operatorname{Sec}=\operatorname{Sec}^{\prime} \cup \operatorname{Sec}^{\prime \prime}$ - те же неравенства с множителем $B(\mathrm{Sec}) \leqslant(k+1)\left(B\left(\mathrm{Sec}^{\prime}\right)+B\left(\mathrm{Sec}^{\prime \prime}\right)\right)$ справа или множителем $B_{0}=B_{0}(\mathrm{Sec}) \leqslant$ $(k+1)\left(B_{0}\left(\mathrm{Sec}^{\prime}\right)+B_{0}\left(\mathrm{Sec}^{\prime \prime}\right)\right)$ соответственно.

Итак, неравенства (6.5) и (6.6) доказаны в случае произвольных замкнутых секторов $E=\operatorname{Sec} \subset D \cup \gamma$. При этом было использовано то, что в неравенствах типа $(6.5),(6.6)$ множители $\Phi(u, \delta)=g\left(\sqrt{U^{-1}(\log \delta)}\right)$ справа зависят от $\delta$ и не зависят от $E$, а коэффициенты $B$ и $B_{0}$ не зависят от $\delta$, но зато зависят от $E$.

J4) Обобщим неравенства (6.5), (6.6), доказанные выше для компактов-секторов $E=\mathrm{Sec}$, на произвольные компакты $E \subset D \cup \gamma$.

Сначала для каждого числа $r \in(0,1)$ и каждой замкнутой дуги $\bar{\sigma} \subset C=\partial D$ построим выпуклую жорданову область $G(r, \sigma) \subset D$ специального вида, содержащую $о \bar{\sigma} \subset \gamma$ радиуса $1-r$ с центром в нуле, имеющую в составе своей границы дугу $\bar{\sigma} \subset \gamma$. Для этого из начала $\zeta_{1}$ дуги $\bar{\sigma}$ и из ее конца $\zeta_{2}$ проведем по одной касательной (соответственно, $T_{1}$ и $\left.T_{2}\right)$ к окружности $C(0,1-r)=\partial D(0,1-r)$ так, чтобы они не пересекались между собой внутри $D$ и чтобы перечисленные ниже дуги и отрезки, проходимые в указанном порядке, образовали выпуклую замкнутую жорданову кривую $\partial G(r, \sigma) \subset D \cup \bar{\sigma}$, ограничивающую выпуклую жорданову подобласть $G(r, \sigma)$ круга $D$, причем $G(r, \sigma) \supset D(0,1-r)$. Вот это перечисление:

1) дуга $\bar{\sigma}=\smile \zeta_{1} \zeta_{2}$, проходимая против часовой стрелки;

$2)$ отрезок $\left[\zeta_{2}, t_{2}\right]$, где $t_{m}$ - точка касания прямой $T_{m}$ с $C(0,1-r)$;

$3)$ дуга $\smile t_{2} t_{1} \subset C(0,1-r)$, проходимая против часовой стрелки;

4) отрезок $\left[t_{1}, \zeta_{1}\right]$.

Пусть теперь $E$ - некоторый компакт, удовлетворяющий условиям теоремы $6.2, \sigma$ и $\sigma^{*}$ - дуги на $\gamma, E \cap \partial D \subset \sigma, \bar{\sigma} \subset \sigma^{*}, \overline{\sigma^{*}} \subset \gamma$. По доказанному выше на секторе $\mathrm{Sec}^{*}:=\overline{\operatorname{Sec}\left(\sigma^{*}\right)}$, опирающемся на дугу $\overline{\sigma^{*}}$, выполняются неравенства (6.5) и (6.6) (с заменой в них $E$ на $\left.\operatorname{Sec}^{*}\right)$. Фиксируем число $r=r(E) \in(0,1)$ столь малым, чтобы $E \subset \overline{G(r, \sigma)}$ и чтобы при $B(r):=\{z: 1-r \leqslant|z| \leqslant 1\}$ выполнялось включение $\overline{G(r, \sigma)} \cap B(r) \subset \mathrm{Sec}^{*}$.

Оценим модуль непрерывности функции $\psi(z)$ на выпуклой замкнутой области $\overline{G(r, \sigma)} \supset E$. Как и выше (см. пп. а)-d) в разделе J1) доказательства) рассмотрим все возможные случаи взаимного расположения точек $z, t \in \overline{G(r, \sigma)}$.

i) Если $z, t \in \overline{D(0,1-r)}$, то $|\psi(z, t)| \leqslant M|z-t|$, где $M=\max \left\{\left|\psi^{\prime}(\zeta)\right|: \zeta \in\right.$ $\overline{D(0,1-r)}\}$. При таких $z, t$ имеем

$$
|z-t|<2, \quad|z-t|=2 \frac{|z-t|}{2}<2 \sqrt{\frac{|z-t|}{2}}=\sqrt{2|z-t|} .
$$

Используя (4.6) (можно использовать и (4.14) и (4.22)), отсюда получаем неравенство

$$
|\psi(z, t)| \leqslant M B^{\prime} g\left(\sqrt{U^{-1}(1 ; \log |z-t|)}\right),
$$

где $B^{\prime}$ не зависит от $z, t \in D(0,1-r)$.

ii) Если $z, t \in \overline{G(r, \sigma)} \cap B(r)$, то $z, t \in \mathrm{Sec}^{*}$, так что $|\psi(z, t)|$ оценивается правой частью неравенства (6.5) (при $\Gamma \in J(g, \varepsilon)$ и $|z-t| \leqslant \delta$ ) либо правой частью неравенства (6.6) (при $\Gamma \in J_{0}(h, \eta)$ и $\left.|z-t| \leqslant \delta\right)$.

iii) Если $z \in \overline{D(0,1-r)}, t \in \overline{G(r, \sigma)} \cap B(r)$ и $|z-t| \leqslant \delta$, обозначим через $t^{\prime}$ общую точку отрезка $[z, t]$ и окружности $C(0,1-r)$. Тогда $\left[z, t^{\prime}\right] \subset \overline{D(0,1-r)}$ 
и $\left|z-t^{\prime}\right| \leqslant \delta$, а $\left[t^{\prime}, t\right] \subset \operatorname{Sec}^{*}$ и $\left|t^{\prime}-t\right| \leqslant \delta$. Из неравенства $|\psi(z, t)| \leqslant\left|\psi\left(z, t^{\prime}\right)\right|+$ $\left|\psi\left(t^{\prime}, t\right)\right|$ и рассмотренных выше пунктов i) и ii) получаем неравенства (6.5) и (6.6) в рассматриваемом случае при $\delta \in[0,2]$. А так как $\omega(\psi, E, \delta) \equiv \omega(\psi, E, 2)$ при $\delta \geqslant 2$ и $\omega(\psi, E, \delta) \leqslant \omega(\psi, \overline{G(r, \sigma)}, \delta)$ при любых $\delta \geqslant 0$, то неравенства $(6.5)$ и (6.6) доказаны в общем случае (см. J2), J3)).

J5) Доказательство неравенства (6.7) проводится по той же схеме, что и только что законченное доказательство неравенства (6.6) - повторяются все рассуждения предыдущих пунктов, касающиеся именно неравенства (6.6). При этом также используется теорема 4.1, но уже со ссылкой на лемму 2.4 при $u(x)=m m_{c}(x):=\pi S(c) x\left(\right.$ см. (4.11)). Так как $m m_{c}(x)=\pi S(c) x \geqslant \pi S_{t}(x)$ (см. лемму 2.4 или формулу (4.11)), то условие (4.3) теоремы 4.1 выполняется. Функции $M M_{c}(1 ; x)$ и $M M_{c}^{-1}(1 ; y)$ легко вычисляются (см. (4.12)):

$$
M M_{c}(1 ; x)=\pi S(c) \log x, \quad M M_{c}^{-1}(1 ; y)=e^{y /(\pi S(c))} .
$$

Доказательство теоремы 6.2 закончено.

ДоКАЗАТЕЛЬСТВо ЗАмЕчАНИЯ 6.2. Как было сказано в этом замечании, нужный пример дают области $G_{s}$ вида $G_{s}:=\left\{w=\psi_{s}(z):|z|<1\right\}$ при малых $s>0$, где $\psi_{s}(z):=|z+1|^{s} e^{i s \arg (z+1)},-\pi / 2<\arg (z+1)<\pi / 2,-$ однозначная и однолистная аналитическая ветвь функции $w=(z+1)^{s}$ в единичном круге $D=\{z:|z|<1\}$. Граница $\psi_{s}(C)(C=\partial D)$ области $G_{s}$ состоит из точки $0=$ $\psi_{s}(-1)$ и открытой аналитической дуги $\psi_{s}(C \backslash\{-1\})$, концы которой сходятся в точке $w=0$, образуя в ней угол величины $\pi s<\pi$. Замыкание открытой дуги $\Gamma_{s}:=\left\{w: w \in \partial G_{s}, \operatorname{Im} w>0, \operatorname{Re} w<1 / 2\right\}$ - это гладкая жорданова дуга. Как таковая она принадлежит классу $J\left(g, \varepsilon_{s}\right)$ с $g(x) \equiv x$ и некоторым $\varepsilon_{s}>0$, а также при каждом $c>1$ - классу $J_{0}\left(h_{c}, \eta_{s}(c)\right)$ с $h_{c}(x) \equiv c x$ и некотором $\eta_{s}(c)>0$. Очевидно, если $\gamma_{s}:=\psi_{s}^{-1}\left(\Gamma_{s}\right)$, то при любом $s \in(0,1)$ верно неравенство

$$
\omega\left(\psi_{s}, \overline{\gamma_{s}}, \delta\right) \geqslant\left|\psi_{s}(z(\delta))-\psi_{s}(-1)\right|=\delta^{s},
$$

справедливое при любом $\delta \in\left[0, \varepsilon_{s}\right] \cap\left[0, \eta_{s}(c)\right]$, если под $z(\delta)$ понимать точку окружности $C$ с $\operatorname{Im} z(\delta)>0$, удаленную от точки $z=-1$ на расстояние $\delta$ : $|z(\delta)+1|=\delta$.

Таким образом, если предположить, что для отображения $w=\psi(z):=\psi_{s}(z)$ круга $D$ на область $G:=G_{s}$ верно неравенство (6.5) при $E=\bar{\gamma}:=\overline{\gamma_{s}}$ с некоторым конечным $B$, то

$$
B g\left(\sqrt{D_{g}^{-1}(\log \delta)}\right) \geqslant \omega(\psi, \bar{\gamma}, \delta) \geqslant \delta^{s}, \quad \delta \in\left[0, \varepsilon_{s}\right] .
$$

Вычислив левую часть этого неравенства при $g(x) \equiv x, x \geqslant 0$ (см. (4.15)), получаем оценку

$$
B \delta^{k} \geqslant \delta^{s}, \quad \delta \in\left[0, \varepsilon_{s}\right], \quad k=\frac{2}{\pi^{2}+2} .
$$

Очевидно, что при любом фиксированном $s \in(0, k)$ это неравенство не выполняется, если $\delta \rightarrow 0$. Справедливость замечания 6.2 доказана.

Теорема 6.3. Пусть $G$ и $Q$ - односвязные ограниченные области плоскости $\mathbb{C}, \Gamma$ и $K$ - открытые достижимые жсордановы дуги их грании, соответственно, причем $\Gamma \in J(g, \varepsilon), K \in J(q, \eta)$. Тогда если $w=f(z)$ - однолистное 
конформное отображение $G$ на $Q$, причем $K=f(\Gamma)$, то для любого компакта $E \subset G \cup \Gamma$ имеем неравенство

$$
\omega(f, E, \delta) \leqslant C^{*} q\left(\sqrt{D_{q}^{-1}\left(2^{-1} \log g(\delta)\right)}\right), \quad \delta \geqslant 0 .
$$

Если при этом $K \in J_{0}(h, \vartheta)$, то

$$
\omega(f, E, \delta) \leqslant C_{0}^{*} q\left(\sqrt{M_{h}^{-1}\left(2^{-1} \log g(\delta)\right)}\right), \quad \delta \geqslant 0,
$$

и если к тому же $h(x) \leqslant c x, x \in[0, \vartheta], c=$ const $\geqslant 1, \vartheta=$ const $>0$, то верно и более точное неравенство

$$
\omega(f, E, \delta) \leqslant C_{1}^{*}(g(\delta))^{\alpha(c) / 2}, \quad \delta \geqslant 0 .
$$

Здесь конечные положительные величины $C^{*}, C_{0}^{*}, C_{1}^{*} u \alpha(c) \leqslant 1$ не зависят от $\delta$, величина $\alpha(c)$ та же, что в (4.20).

ЗАмЕчАниЕ 6.4. Если дуга Г - ляпуновская или обобщенно ляпуновская (см. замечание 3.1), то при условиях теоремы 6.3 справа в неравенствах (6.11), (6.12) можно убрать множитель $2^{-1}$, а в неравенстве (6.13) можно вместо $\alpha(c) / 2$ взять $\alpha(c)$. Если же (обобщенно) ляпуновской является дуга $K$, то (6.11) запишется в следующем виде: $\omega(f, E, \delta) \leqslant C_{2}^{*} \sqrt{g(\delta)}, \delta \geqslant 0$. Наконец, если обе дуги Г и $K$ являются обобщенно ляпуновскими, то отображение $w=f(z)$ непрерывно дифференцируемо на $G \cup \Gamma$.

ДоКАЗАТЕЛЬСТво теОРЕмы 6.3. Эта теорема в основном является следствием теорем 6.1 и 6.2. Действительно, пусть $\zeta=\varphi(z)$ - какое-либо однолистное конформное отображение области $G$ на единичный круг $D, \gamma:=\varphi(\Gamma) \subset C$. Тогда функция $w=\psi(\zeta):=f\left(\varphi^{-1}(\zeta)\right)$ однолистно и конформно отображает круг $D$ на область $Q$, причем $\psi(\gamma)=K$. По теореме 6.1 имеем неравенство $\omega(\varphi, E, \delta) \leqslant A_{1}(E) \sqrt{g(\delta)}, \delta \geqslant 0$ (см. (6.2)). По теореме 6.2 при $E_{1}:=\varphi(E)$ и всех $\delta_{1} \geqslant 0$ верны следующие неравенства:

$$
\begin{array}{ll}
\omega\left(\psi, E_{1}, \delta_{1}\right) \leqslant B q\left(\sqrt{D_{q}^{-1}\left(\log \delta_{1}\right)}\right), & K \in J(q, \eta) \quad(\text { см. }(6.5)), \\
\omega\left(\psi, E_{1}, \delta_{1}\right) \leqslant B_{0} q\left(\sqrt{M_{h}^{-1}\left(\log \delta_{1}\right)}\right), & K \in J_{0}(h, \vartheta) .
\end{array}
$$

При этом в последнем неравенстве можно справа заменить $q(\cdot)$ на $h(\cdot)$ (см. $(6.6))$ и если $h(x) \leqslant c x, x \in[0, \eta], c=$ const $\geqslant 1$, то $\omega\left(\psi, E_{1}, \delta_{1}\right) \leqslant B_{1} \delta_{1}^{\alpha(c)}$ (см. (6.7)).

Далее, из очевидного тождества $f(z) \equiv \psi(\varphi)(z):=\psi(\varphi(z))$ получаем тождество $\omega(f, E, \delta) \equiv \omega(\psi(\varphi), E, \delta)$. Отсюда и из $(6.2)$ легко получаем неравенства

$$
\omega(f, E, \delta) \leqslant \omega\left(\psi, E_{1}, \omega(\varphi, E, \delta)\right) \leqslant \omega\left(\psi, E_{1}, A_{1}(E) \sqrt{g(\delta)}\right) .
$$

Осталось подставить в правую часть неравенства (6.14) приведенные в начале этого доказательства оценки типа (6.5)-(6.7) для $\omega\left(\psi, E_{1}, \delta_{1}\right)$. Однако при этом возникает неувязка с "выносом" величины $A_{1}(E)$ из "глубины" правой части неравенства (6.14) в начало этой части в виде множителя, не зависящего 
от $\delta$. Трудность эту мы обойдем, заменив в (6.14) компакт $E_{1}$ выпуклым компактом $E_{2}:=\overline{G\left(r\left(E_{1}\right), \sigma\right)} \supset E_{1}$ (о построении этого компакта см. выше п. J4) доказательства теоремы 6.2). При этом $E_{2} \subset D \cup \bar{\sigma}$, если в качестве $\sigma$ взять какую-либо дугу на $\gamma$, для которой $\bar{\sigma} \subset \gamma$ и $E_{1} \subset D \cup \bar{\sigma}$. Из выпуклости компакта $E_{2} \supset E_{1}$ и свойства (2.2) модуля непрерывности при $\lambda:=A_{1}(E)$ и любых $\delta \geqslant 0$ получаем из (6.14) неравенство

$$
\omega(f, E, \delta) \leqslant \omega\left(\psi, E_{1}, \lambda \sqrt{g(\delta)}\right) \leqslant(\lambda+1) \omega\left(\psi, E_{2}, \sqrt{g(\delta)}\right) .
$$

Для получения оценок (6.11)-(6.13) для $\omega(f, E, \delta)$ остается подставить в правую часть неравенства (6.15) оценки (6.5)-(6.7) соответственно для $\omega\left(\psi, E_{2}, \sqrt{g(\delta)}\right)$.

Справедливость замечания 6.4 следует из теорем $6.1,6.2$ и замечания 3.1 .

В заключение я хочу выразить искреннюю благодарность моим ученикам, особенно П. А. Бородину и О.Н. Косухину, за существенную помощь при подготовке этой работы к печати.

ЗАмЕчАниЕ 6.5. Недавно была опубликована статья [18], в которой, в частности, доказано, что каждое конформное отображение $w=\varphi(z)$ ограниченной выпуклой области $G$ на круг $D$ имеет ограниченную производную $\varphi^{\prime}(z)$ на $\bar{G}$ (так что $\varphi \in \operatorname{Lip} 1$ на $\bar{G}$ ), а также найден критерий непрерывности $\varphi^{\prime}(z), z \in \bar{G}$, в точках $z_{0} \in \partial G$. Отметим, что выпуклые кривые - это, так сказать, "простые" кривые Лаврентьева (см. введение в настоящей статье).

\section{Список литературы}

[1] O. D. Kellogg, "Harmonic functions and Green's integral", Trans. Amer. Math. Soc., 13:1 (1912), 109-132.

[2] S.E. Warschawski, "On the higher derivatives at the boundary in conformal mapping", Trans. Amer. Math. Soc., 38, 1935, 310-340.

[3] S.E. Warschawski, "On differentiability at the boundary in conformal mapping", Proc. Amer. Math. Soc., 12:4 (1961), 614-620.

[4] Е. П. Долженко, "Замечания о модуле непрерывности конформного отображения круга на жорданову область", Матем. заметки, 60:2 (1996), 176-184; англ. пер.: E. P. Dolzhenko, "Some remarks on the modulus of continuity of a conformal mapping of the disk onto a Jordan domain", Math. Notes, 60:2 (1996), 130-136.

[5] Е. П. Долженко, "О конформных отображениях жордановых областей”, Вестн. Моск. ун-та. Сер. 1. Матем., мех., 1999, № 4, 66-68; англ. пер.: E. P. Dolzhenko, "On conformal mappings of Jordan domains", Moscow Univ. Math. Bull., 54:4 (1999), $47-49$.

[6] E. P. Dolzhenko, "On modules of continuity of conformal mappings of arbitrary Jordan domains", J. Math. Sci. (New York), 108:3 (2002), 411-419.

[7] Е. П. Долженко, "О степени "гладкости" конформных отображений жордановых областей с негладкими границами, Комплексный анализ и теория потенциала", Труды Украинского матем. конгресса 2001 (Киев, Украина, 2001), Ин-т матем. НАН Украины, Киев, 2003, 25-33.

[8] Е.П. Долженко, "О граничной гладкости конформных отображений областей с негладкими границами", Докл. РАН, 415:2 (2007), 155-159; англ. пер.: E.P. Dolzhenko, "On the boundary smoothness of conformal mappings between domains with nonsmooth boundaries", Dokl. Math., 76:1 (2007), 514-518. 
[9] Г. М. Голузин, Геометрическая теория функиий комплексного переменного, Наука, М., 1966; англ. пер.: G. M. Goluzin, Geometric theory of functions of a complex variable, Transl. Math. Monogr., 26, Amer. Math. Soc., Providence, RI, 1969.

[10] М. А. Лаврентьев, "О непрерывности однолистных функций в замкнутых областях", Докл. АН СССР, 4:5 (1936), 207-209.

[11] Г. Д. Суворов, Обобщенный "приниип длины и площади" в теории отображений, Наукова думка, Киев, 1985.

[12] П. М. Тамразов, "Контурные и телесные структурные свойства голоморфных функций комплексного переменного", УМН, 28:1 (1973), 131-161; англ. пер.: P. M. Tamrazov, "Contour and solid structure properties of holomorphic functions of a complex variable", Russian Math. Surveys, 28:1 (1973), 141-173.

[13] В. Бляшке, Круг и шар, Наука, М., 1967; пер. с нем.: W. Blaschke, Kreis und Kugel, de Gruyter, Berlin, 1956.

[14] П. Урысон, "Зависимость между средней шириной и объемом выпуклых тел в $n$-мерном пространстве", Матем. сб., 31:3-4 (1924), 477-486.

[15] С. Сакс, Теория интеграла, ИЛ, М., 1949; пер. с англ.: S. Saks, Theory of the integral, Dover Publ., New York, 1937.

[16] И. И. Привалов, Граничные свойства аналитических функиий, ГИТТЛ, М.-Л., 1950.

[17] Е. П. Долженко, "Гладкость гармонических и аналитических функций в граничных точках области", Изв. АН СССР. Сер. матем., 29:5 (1965), 1069-1084.

[18] Е. П. Долженко, С. В. Колесников, "О поведении конформных отображений областей вблизи их выпуклых граничных дуг", Матем. заметки, 90:4 (2011), 501-516; E. P. Dolzhenko, S. V. Kolesnikov, "The behavior of conformal maps of domains near convex boundary arcs", Math. Notes, 90:4 (2011), 485-497.

\section{Е. П. Долженко (Е. P. Dolzhenko)}

Механико-математический факультет

Московского государственного университета

им. М. В. Ломоносова

E-mail: eugen@ngcom.ru
Поступила в редакцию 03.11.2009 и 24.02.2011 